\title{
The earliest phases of high-mass star formation: a 3 square degree millimeter continuum mapping of Cygnus $X^{\star, \star \star}$
}

\author{
F. Motte ${ }^{1,2}$, S. Bontemps ${ }^{3}$, P. Schilke ${ }^{4}$, N. Schneider ${ }^{1,5}$, K. M. Menten ${ }^{4}$, and D. Broguière ${ }^{6}$ \\ ${ }^{1}$ Laboratoire AIM, CEA/DSM - CNRS - Université Paris Diderot, DAPNIA/Service d'Astrophysique, Bât. 709, CEA-Saclay, \\ 91191 Gif-sur-Yvette Cedex, France \\ e-mail: motte@cea.fr \\ 2 California Institute of Technology, Downs Laboratory of Physics, Mail Stop 320-47, 1200 E California Blvd, Pasadena, \\ CA 91125, USA \\ 3 OASU/LAB-UMR 5804, CNRS, Université Bordeaux 1, 2 rue de l'Observatoire, BP 89, 33270 Floirac, France \\ ${ }^{4}$ Max-Planck-Institut für Radioastronomie, Auf dem Hügel 69, 53121 Bonn, Germany \\ 5 I. Physik. Institut, Universität Köln, 50937 Köln, Germany \\ 6 IRAM, 300 rue de la Piscine, 38406 Saint Martin d'Hères, France
}

Received 10 May 2007/ Accepted 20 July 2007

\section{ABSTRACT}

\begin{abstract}
Aims. Our current knowledge of high-mass star formation is mainly based on follow-up studies of bright sources found by IRAS, and is thus biased against its earliest phases, inconspicuous at infrared wavelengths. We therefore started searching, in an unbiased way and in the closest high-mass star-forming complexes, for the high-mass analogs of low-mass pre-stellar cores and class 0 protostars. Methods. We have made an extensive $1.2 \mathrm{~mm}$ continuum mosaicing study of the Cygnus $\mathrm{X}$ molecular cloud complex using the MAMBO cameras at the IRAM $30 \mathrm{~m}$ telescope. The $\sim 3^{\circ{ }^{2}}$ imaged areas cover all the high-column density $\left(A_{\mathrm{V}} \geq 15 \mathrm{mag}\right)$ clouds of this nearby $(\sim 1.7 \mathrm{kpc})$ cloud complex actively forming OB stars. We then compared our millimeter maps with mid-infrared images, and have made $\mathrm{SiO}(2-1)$ follow-up observations of the best candidate progenitors of high-mass stars.

Results. Our complete study of Cygnus $\mathrm{X}$ with $\sim 0.09 \mathrm{pc}$ resolution provides, for the first time, an unbiased census of massive young stellar objects. We discover 129 massive dense cores $\left(F W H M\right.$ size $\sim 0.1 \mathrm{pc}, M_{1.2} \mathrm{~mm}=4-950 M_{\odot}$, volume-averaged density $\left.\sim 10^{5} \mathrm{~cm}^{-3}\right)$, among which $\sim 42$ are probable precursors of high-mass stars. A large fraction of the Cygnus X dense cores $(2 / 3$ of the sample) remain undetected by the $M S X$ satellite, regardless of the mass range considered. Among the most massive ( $\left.\geq 40 M_{\odot}\right)$ cores, infrared-quiet objects are driving powerful outflows traced by $\mathrm{SiO}$ emission. Our study qualifies 17 cores as good candidates for hosting massive infrared-quiet protostars, while up to 25 cores potentially host high-luminosity infrared protostars. We fail to discover the high-mass analogs of pre-stellar dense cores $\left(\sim 0.1 \mathrm{pc},>10^{4} \mathrm{~cm}^{-3}\right)$ in Cygnus X, but find several massive starless clumps $\left(\sim 0.8 \mathrm{pc}, 7 \times 10^{3} \mathrm{~cm}^{-3}\right)$ that might be gravitationally bound.

Conclusions. Since our sample is derived from a single molecular complex and covers every embedded phase of high-mass star formation, it gives the first statistical estimates of their lifetime. In contrast to what is found for low-mass class 0 and class I phases, the infrared-quiet protostellar phase of high-mass stars may last as long as their better-known high-luminosity infrared phase. The statistical lifetimes of high-mass protostars and pre-stellar cores $\left(\sim 3 \times 10^{4} \mathrm{yr}\right.$ and $\left.<10^{3} \mathrm{yr}\right)$ in Cygnus $\mathrm{X}$ are one and two order(s) of magnitude smaller, respectively, than what is found in nearby, low-mass star-forming regions. We therefore propose that high-mass pre-stellar and protostellar cores are in a highly dynamic state, as expected in a molecular cloud where turbulent processes dominate.
\end{abstract}

Key words. dust, extinction - H II regions - ISM: structure - stars: formation - submillimeter

\section{Introduction}

High-mass $\left(\mathrm{OB},>8 M_{\odot}\right)$ stars, though few in number, play a major role in the energy budget of galaxies. Our current understanding of their formation, however, remains very schematic, especially concerning the earliest phases of the process. High-mass stars are known to form in dense cores within molecular cloud complexes, by accretion (Yorke \& Sonnhalter 2002; Krumholz et al. 2007) and/or coalescence (e.g. Bonnell et al. 2001). The copious UV flux emitted by a newly-formed central star heats

* Tables 1,2, Figs. 10-15, and Appendices A and B are only available in electronic form at http://www . aanda.org

$\star \star$ Fits images associated with Figs. $2 \mathrm{a}-\mathrm{c}$ and catalogs built from Tables 1,2 are only available in electronic form at the CDS via anonymous ftp to cdsarc.u-strasbg. fr $(130.79 .128 .5)$ or via http://cdsweb.u-strasbg.fr/cgi-bin/qcat?]/A+A/476/1243 and ionizes its parental molecular cloud, leading to the formation and development of a hot core (e.g. Helmich \& van Dishoek 1997) and afterwards an H II region (see a review by Churchwell 1999).

In the past few years, the progenitors of ultracompact $\mathrm{H}$ II (UCH II) regions (sources that have not yet begun to ionize their environment in a detectable way) have been searched among IRAS point sources. The criteria generally used aim at selecting high-luminosity $\left(>10^{3} L_{\odot}\right)$ stellar embryos embedded in a massive envelope and associated with hot gas but no UCH II region (see Kurtz et al. 2000). In fact, most surveys focussed on red IRAS sources with colors satisfying the Wood \& Churchwell (1989) criteria (originally set for selecting UCH II regions) but not detected in radio centimeter surveys. The presence of an envelope around the IRAS sources is confirmed by their association with high-density gas, and the existence of hot gas by 
the detection of a hot core and water or methanol masers (e.g. Bronfman et al. 1996; Plume et al. 1997). In this way, studies by e.g., Molinari et al. (2000) and Sridharan et al. (2002) have identified massive young stellar objects harboring high-luminosity infrared protostars (see also Mueller et al. 2002; Faúndez et al. 2004).

Such surveys are biased against younger and, thus probably colder, massive young stellar objects which could be inconspicuous in the mid-infrared bands of IRAS and the Midcourse Space Experiment (MSX). These high-mass analogs of low-mass class 0 protostars and pre-stellar cores (cf. André et al. 2000) should be best detected via far-infrared to (sub)millimeter dust continuum and high-density molecular line tracers. Serendipitous discoveries of a few infrared-quiet massive young stellar objects have been made in submillimeter continuum maps (e.g. Molinari et al. 1998; Motte et al. 2003; Sandell \& Sievers 2004; Hill et al. 2005) and as absorption regions in mid-infrared images (often called "infrared dark clouds", e.g. Carey et al. 2000; Thompson et al. 2005; Rathborne et al. 2006). These studies suggest that, in the high-mass star formation process also, infrared-quiet phases must exist and be associated with significant populations of young protostars and/or pre-stellar cores. The lack of statistics, homogeneity, and angular resolution of studies such as the above, however, prevents the determination of the basic characteristics of these infrared-quiet objects.

To make significant progress, one needs to search in a systematic way for the earliest phases of high-mass star formation in nearby complexes. One of the most appropriate methods is to survey entire molecular cloud complexes in which high-mass stars are forming, using tracers of high-density clouds and protostellar activity signatures. We therefore started a multitracer study of Cygnus X, which is one of the richest molecular and $\mathrm{H}$ II complexes located at less than $3 \mathrm{kpc}$ from the Sun. The molecular cloud complex is massive $\left(4 \times 10^{6} M_{\odot}\right)$ and extends over $\sim 100 \mathrm{pc}$ in diameter (cf. Leung \& Thaddeus 1992). Found to be part of the local spiral arm, this complex has been located at $1.7 \mathrm{kpc}$ from the Sun by the recent ${ }^{13} \mathrm{CO}$ study of Schneider et al. (2006). Cygnus $\mathrm{X}$ has a rich collection of $\mathrm{H}$ II regions (see centimeter free-free emission maps by Wendker et al. 1991, and infrared images from $M S X$ presented in Figs. 7-9 of Schneider et al. 2006), indicating recent high-mass star formation. It is associated with several $\mathrm{OB}$ associations (see Uyaniker et al. 2001, and references therein), amongst them one of the largest in our Galaxy (Cyg OB2, cf. Knödlseder 2000). It also contains several well-known massive young stellar objects like DR21, DR21(OH), AFGL 2591, S106-IR, and W75N (e.g. Smith et al. 1998; van der Tak et al. 1999; Schneider et al. 2002; Shepherd et al. 2004). Small parts of the Cygnus X cloud complex have already been observed in (sub)millimeter continuum emission (e.g. Chandler et al. 1993a; Richer et al. 1993; Vallée \& Fiege 2006; Davis et al. 2007).

The present paper tackles the following key questions: do high-mass pre-stellar cores exist? What are the lifetimes of high-mass pre-stellar cores, infrared-quiet protostars, and highluminosity infrared protostars? What are the main physical processes leading from pre-stellar objects to high-mass protostars? We report an extensive $1.2 \mathrm{~mm}$ continuum study of the Cygnus $\mathrm{X}$ complex complemented by $\mathrm{SiO}(2-1)$ follow-up observations of the best candidate progenitors of high-mass stars. From the MAMBO-2 imaging $\left(\sim 3^{\circ^{2}}\right)$ of the entire complex presented in Sect. 2, we make a complete census of the compact cloud fragments and larger-scale structures (Sect. 3). We determine the main characteristics of these new millimeter sources in Sect. 3,

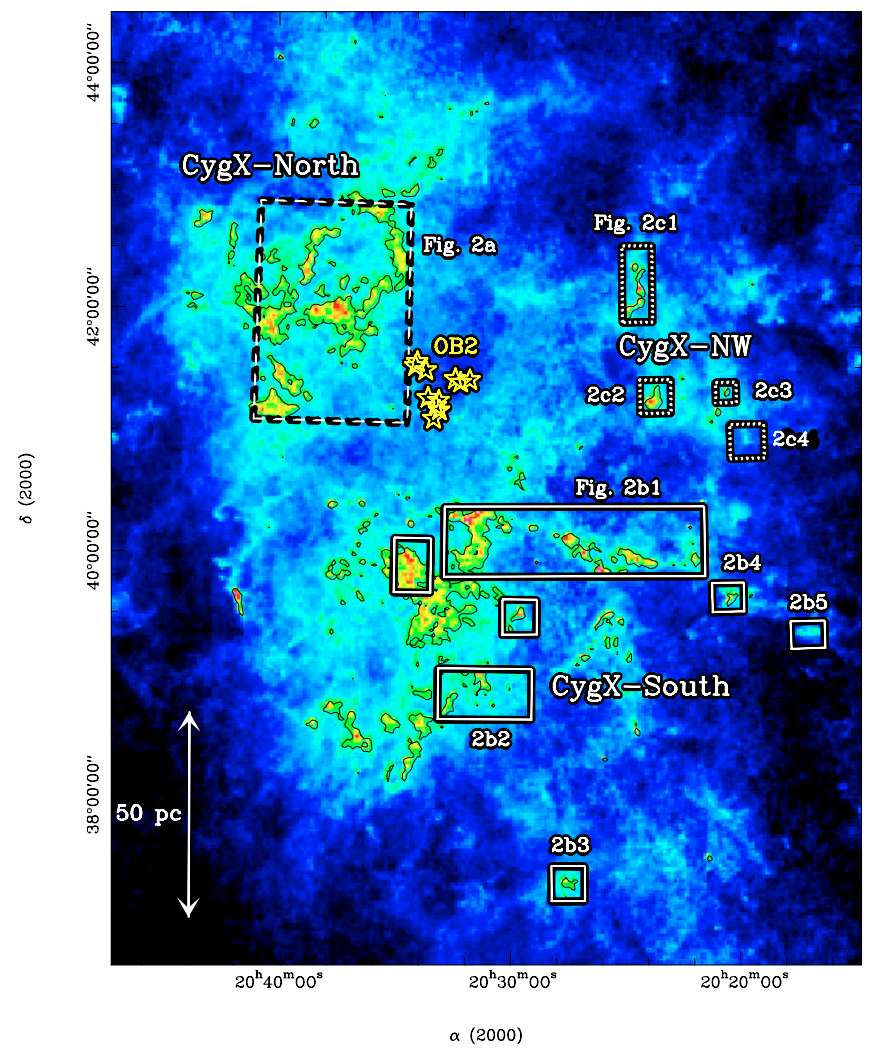

Fig. 1. Extinction map of the Cygnus $\mathrm{X}$ complex derived by Bontemps et al. (in prep.) from the stellar reddening of background stars in $J H K$, using the 2MASS database and a pixel size of $1.3^{\prime}$. The high-extinction regions are yellow and outlined by the 15 mag contour level. The three molecular cloud ensembles identified by Schneider et al. (2006) are labeled and the nearby Cygnus OB2 association is indicated. The fields mapped with MAMBO or MAMBO-2 are schematically outlined and add up to $\sim 3^{\circ^{2}}$.

and search for signposts of protostellar activity including $\mathrm{SiO}$ emission (a tracer of outflow activity) in Sect. 4. Section 5 identifies 129 massive dense cores, among which 42 are probable precursors of high-mass stars. This unbiased sample of young embedded massive stars reveals 17 dense cores that are excellent candidates for harboring high-mass protostars in their infrared-quiet phase. The present paper also gives the first statistical results on the lifetime of high-mass protostars and prestellar cores. Finally, Sect. 6 summarizes our conclusions, and Appendices A and B describe our data reduction and source extraction techniques.

\section{Observations and data reduction}

\subsection{Imaging the entire molecular complex}

The Cygnus X molecular cloud complex covers up to $\sim 30^{\circ}$ (see Fig. 1 of Schneider et al. 2006) but the sites of high-mass star formation are found in its highest-density parts. We therefore used an infrared extinction map produced from $2 \mathrm{MASS}^{1}$ data (cf. Fig. 1 and Bontemps et al. in prep.) to select the high-column density ( $A_{\mathrm{V}} \geq 15 \mathrm{mag}$ ) clouds of Cygnus $\mathrm{X}$ to be mapped in millimeter continuum emission. Figure 1 and Schneider et al. (2006) show that the Cygnus X molecular complex contains several ensembles of dense molecular clouds which extend over 50 parsecs. In the following, we use the name "CygX-North" for the

1 The Two Micron All Sky Survey was performed by the University of Massachussets and IPAC/Caltech and funded by NASA and NSF. 
north-eastern group of clouds which harbors the well-known sources DR21 and W75N, "CygX-South" for the southern region containing AFGL 2591 and S106-IR, and "CygX-NW" for the north-western filamentary clouds. The mappings necessary to cover the high-column density clouds of Cygnus $\mathrm{X}$ are outlined in Fig. 1 and cover a total area of $\sim 3^{\circ^{2}}$.

\subsection{Dust continuum observations at $1.2 \mathrm{~mm}$}

The Cygnus $\mathrm{X}$ molecular complex was imaged at $1.2 \mathrm{~mm}$ with the MPIfR bolometer arrays installed at the IRAM ${ }^{2}$ $30 \mathrm{~m}$ telescope at Pico Veleta (Spain). The passband of the MPIfR bolometers has an equivalent width of $\approx 70 \mathrm{GHz}$ and is centered at $v_{\text {eff }} \approx 240 \mathrm{GHz}$ (Kreysa et al. 1998). The MAMBO (37 channels) camera was used in February-April 1999, January-March 2000, and December 2000-May 2001 for a total integration time of $\sim 30 \mathrm{~h}$. The MAMBO-2 (117 channels) camera was used for $\sim 33 \mathrm{~h}$ as part of the observing pools organized by IRAM between November 2002 and March 2003. A total of 142 large on-the-fly maps were taken using a fast-mapping technique similar to that described by Teyssier \& Sievers (1998). These maps consist of a series of rows scanned in azimuth with a velocity of $8^{\prime \prime} / \mathrm{s}$ and spaced by $22^{\prime \prime}$ (respectively $46^{\prime \prime}$ ) steps in elevation when using MAMBO or MAMBO-2. The typical azimuthal size of individual maps is $\sim 7.5^{\prime}$ with MAMBO versus $\sim 11^{\prime}$ with MAMBO-2. The signal of each bolometer is modulated by the secondary mirror which is wobbling with a frequency of $2 \mathrm{~Hz}$ and a throw in azimuth of $70^{\prime \prime}$. The resulting dual-beam maps were reduced with the IRAM software for bolometer-array data (NIC; cf. Broguière et al. 1995) using the EKH restoration algorithm (Emerson et al. 1979) and a skynoise reduction technique developed by us (see Appendix A). The imaging of each requested field was obtained by combining partially overlapping restored on-the-fly maps.

The main beam was measured and found to have a $H P B W$ size of $\sim 11^{\prime \prime}$ using Uranus and Mars. The absolute pointing of the telescope was found to be accurate to within $\sim 5^{\prime \prime}$. The data were all taken in winter but with heterogeneous weather conditions. The zenith atmospheric optical depth varied between 0.1 and 0.5 and the skynoise level was low to high (correlation factor from 0.2 to 1 ). The resulting rms noise in individual maps is $\sigma=10-150 \mathrm{mJy}^{-1}$ beam $^{-1}$, reduced to $\sigma \sim 20 \mathrm{mJy} \mathrm{beam}^{-1}$ after reduction of the skynoise (see Appendix A). The final mosaics all have $\sigma<20$ mJy beam $^{-1} \mathrm{rms}$ with a median rms of $\sigma \sim 15 \mathrm{mJy} \mathrm{beam}^{-1}$. Uranus and Mars were also used for flux calibration and the overall absolute calibration uncertainty is estimated to be $\sim 20 \%$.

\section{3. $\mathrm{SiO}(2-1)$ observations}

Pointed observations in the $\mathrm{SiO} v=0 J=2 \rightarrow 1$ transition were performed for 40 millimeter continuum sources listed in Table 1 in September 2003 and July 2004 with the IRAM $30 \mathrm{~m}$ telescope. We used the A100 (B100) SIS receiver and the VESPA autocorrelator with a frequency resolution of $40 \mathrm{kHz}$ that is equivalent to $0.135 \mathrm{~km} \mathrm{~s}^{-1}$ at $86.847 \mathrm{GHz}$. The system temperature for both observing runs was between 110 and $130 \mathrm{~K}$ and the average rms for each data set is $0.03 \mathrm{~K}$ on a main beam brightness temperature scale, taking an efficiency $\eta_{\mathrm{MB}}$ of 0.78 . The angular resolution of the telescope at $87 \mathrm{GHz}$ is $29^{\prime \prime}$. All observations were performed in position-switching mode with off-positions a few arcminutes away in azimuth. Pointing was

${ }^{2}$ IRAM is supported by INSU/CNRS (France), MPG (Germany) and IGN (Spain). checked regularly and was accurate to within $3^{\prime \prime}$. Standard calibration sources were observed regularly and were consistent within $10 \%$. The data were reduced with the IRAM software for spectral lines (CLASS), applying first order baselines and averaging individual spectra with rms weighting.

\section{Analysis of the $1.2 \mathrm{~mm}$ mapping of Cygnus $X$}

\subsection{Dust continuum images}

The $1.2 \mathrm{~mm}$ continuum images are presented in Figs. $2 \mathrm{a}-\mathrm{c}$ with zooms in the left-hand parts of Figs. 3, 4 (see also Figs. 12-14 which are only available electronically). The dense molecular gas sometimes follows filamentary structures (well observed in the ${ }^{13} \mathrm{CO}(1-0)$ images of Schneider et al. in prep.) and displays many compact $(\sim 0.1 \mathrm{pc})$ fragments. We make a complete census of these $1.2 \mathrm{~mm}$ fragments in Sect. 3.2 and a similar but less complete census of larger-scale $(\sim 1 \mathrm{pc})$ cloud structures in Sect. 3.3.

The MAMBO-2 mosaics are sensitive to spatial scales ranging from $\sim 0.09 \mathrm{pc}$ (corresponding to an $H P B W$ of $11^{\prime \prime}$ at $1.7 \mathrm{kpc}$ ) to $\sim 5$ pc (i.e. the mean azimuth extent of individual maps $\sim 10^{\prime}$ at $1.7 \mathrm{kpc}$ ). The resulting spatial dynamic is $\sim 55$, larger than that of other (sub)millimeter dust continuum observations made with SCUBA at the JCMT, SIMBA at the SEST, or BOLOCAM at the CSO. Therefore, large fast-mapping images with MAMBO-2 are currently the best tool to study the density structure of molecular clouds with high spatial resolution. Given the dynamic range in the MAMBO-2 image (within a 11" beam, cold dust emission ranges from $\sigma \sim 15 \mathrm{mJy}_{\text {beam }}^{-1}$ to $6300 \mathrm{mJy} \mathrm{beam}^{-1}$ ), the Cygnus $\mathrm{X}$ complex is probed from $N_{\mathrm{H}_{2}} \sim 9 \times 10^{21} \mathrm{~cm}^{-2}$ to $2 \times 10^{24} \mathrm{~cm}^{-2}$, or similarly from $A_{\mathrm{V}} \sim 10 \mathrm{mag}$ to $2100 \mathrm{mag}$ (i.e. with a column density dynamic of more than 200). The above estimate uses Eq. (1) from Motte et al. (1998) with dust temperatures of $20 \mathrm{~K}$ and dust opacities of $\kappa_{1.2 \mathrm{~mm}}=0.005$ and $0.01 \mathrm{~cm}^{2} \mathrm{~g}^{-1}$ for the lower and higher density cloud structures, respectively.

Due to the spatial filtering of cloud structures larger than clumps (i.e. $>5$ pc, see Appendix A) in MAMBO-2 images, the total mass detected in millimeter continuum emission is 10 to 20 times smaller $\left(37 \times 10^{3} M_{\odot}\right.$ in CygX-North and $26 \times$ $10^{3} M_{\odot}$ in CygX-South) than that measured in the CO survey of Schneider et al. (2006).

\subsection{Census of compact cloud fragments in Cygnus $X$}

Since our main goal is to investigate the best potential sites of high-mass star formation, we first focus on the small-scale cloud fragments observed in our MAMBO-2 images. As expected, larger-scale $(\sim 1 \mathrm{pc})$ cloud structures generally have a moderate volume-averaged density $\left(\sim 5 \times 10^{3} \mathrm{~cm}^{-3}\right.$ for a $1.2 \mathrm{~mm}$ peak flux of $5 \sigma=75 \mathrm{mJy} \mathrm{beam}^{-1}$ over a diameter of $1 \mathrm{pc}$, when a $20 \mathrm{~K}$ dust temperature and a $\kappa_{1.2 \mathrm{~mm}}=0.01 \mathrm{~cm}^{2} \mathrm{~g}^{-1}$ dust mass opacity are assumed, cf. Eqs. (1) and (2)) and will therefore be less likely to form high-mass stars in the near future. We apply the source extraction technique developed by Motte et al. (2003) which uses a multi-resolution analysis (Starck \& Murtagh 2006) and the Gaussclumps program (Stutzki \& Güsten 1990; Kramer et al. 1998). We first identify 129 compact $(<1$ pc) cloud fragments above the median $5 \sigma=75 \mathrm{mJy}^{\text {beam }}{ }^{-1}$ level in the MAMBO-2 maps, where spatial scales larger than $1 \mathrm{pc}$ have been filtered out (see e.g. Fig. 11b). We then precisely derive the $1.2 \mathrm{~mm}$ characteristics of each fragment by fitting a 2D-Gaussian in the image where its local background (or surrounding large-scale emission) has been entirely removed (see Appendix B for more details). 


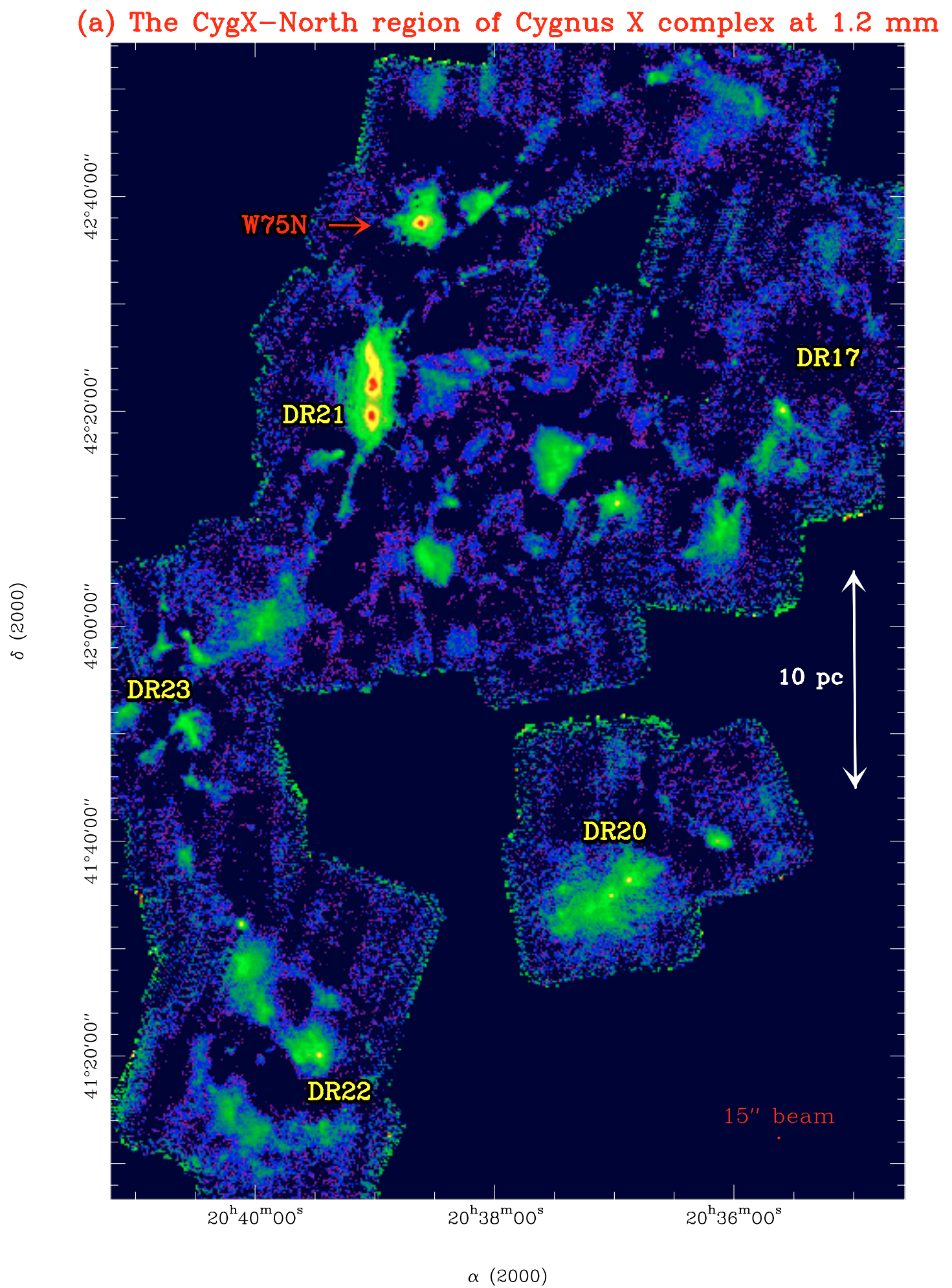

Fig. 2. Millimeter continuum imaging of the Cygnus $X$ molecular cloud complex obtained with the MAMBO and MAMBO-2 cameras installed at the IRAM $30 \mathrm{~m}$ telescope. These $1.2 \mathrm{~mm}$ maps have been smoothed to an effective angular resolution of $15^{\prime \prime}$, allowing a sensitivity of $0.1-5 \mathrm{pc}$ cloud structures. The main radio sources (Downes \& Rinehart 1966) and a few well-known sources are indicated as reference marks. a) The CygX-North region (see Fig. 1 for its location): maximum flux is $\sim 8500 \mathrm{mJy}^{-1} \mathrm{~mm}^{-1}$ (color scale is saturated beyond $500 \mathrm{mJy} \mathrm{beam}^{-1}$ ) and $\mathrm{rms}$ noise level is $\sigma=10-20$ mJy beam $^{-1}$.

The compact fragments extracted here are marked and labeled in the left-hand parts of Figs. 3, 4 and Figs. 12-14. Table 1, which is only available electronically, lists the 129 fragments discovered in Cygnus $\mathrm{X}$ and gives their $1.2 \mathrm{~mm}$ basic characteristics as estimated above. The fragments are listed in increasing
RA coordinate for each region. They are numbered (Col. 1) and named after their 2000 equatorial coordinates (Col.2). Their $1.2 \mathrm{~mm}$ peak flux density (Col. 3), their FWHM size (Col. 4), and integrated flux (Col. 5) are measured assuming a Gaussian shape. In Col.9, we list previous names given to strong millimeter 

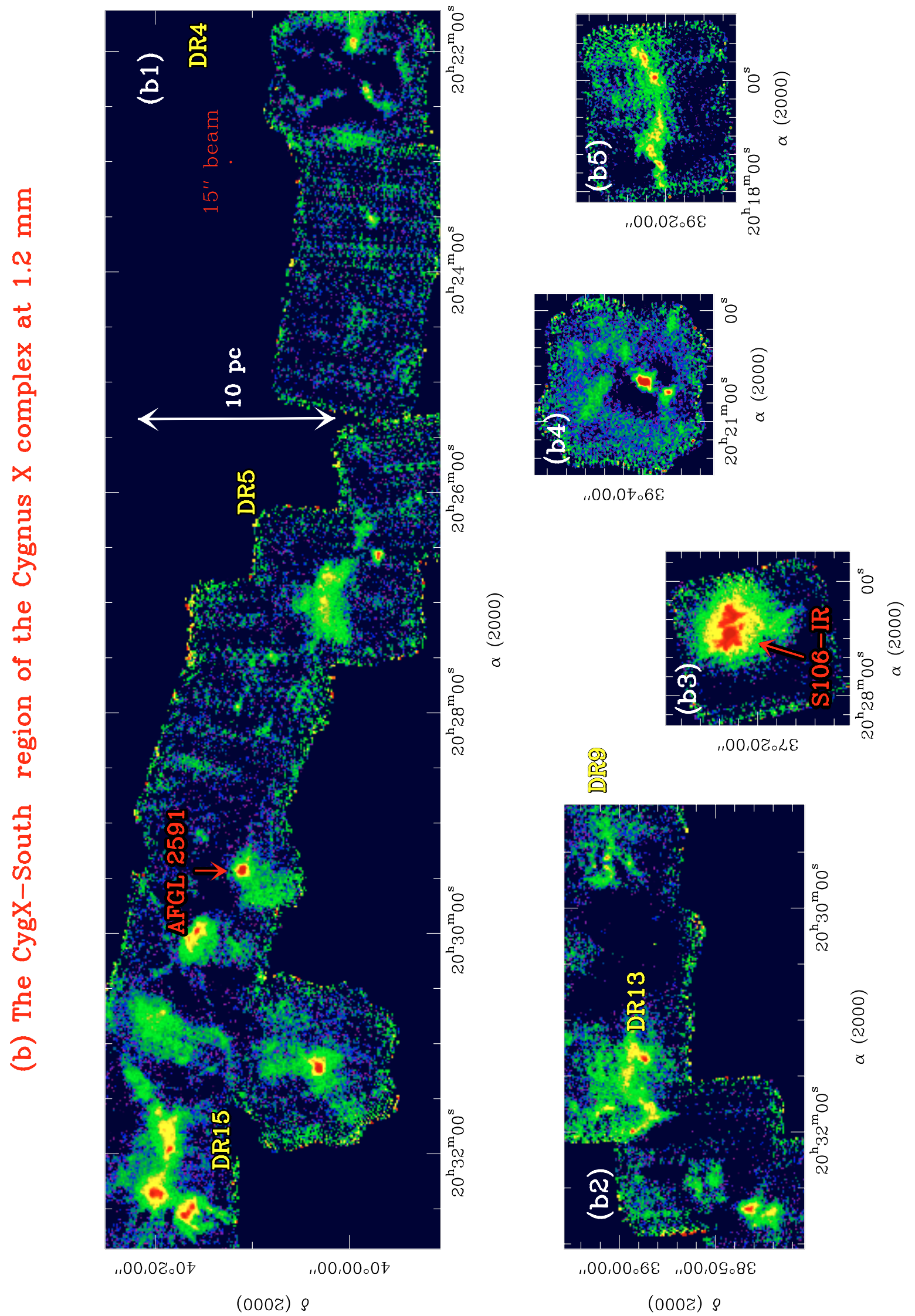

Fig. 2. continued. b) The CygX-South region: maximum flux is $\sim 8500 \mathrm{mJy}_{\text {beam }}^{-1}$ (color scale is saturated beyond $500 \mathrm{mJy}^{-1} \mathrm{beam}^{-1}$ ) and $\mathrm{rms}^{-1}$ noise levels are 9-18 $\mathrm{mJy}_{\text {beam }}{ }^{-1}$ in b1), $\sim 14 \mathrm{mJy} \mathrm{beam}^{-1}$ in b2), $\sim 14 \mathrm{mJy}_{\text {beam }}^{-1}$ in b3), $\sim 13 \mathrm{mJy} \mathrm{beam}^{-1}$ in b4), and $\sim 15 \mathrm{mJy} \mathrm{beam}^{-1}$ in b5). Note that the MAMBO-2 maps associated with DR12 and DR6 in Fig. 1 are ignored since they do not display any compact and dense gas. 


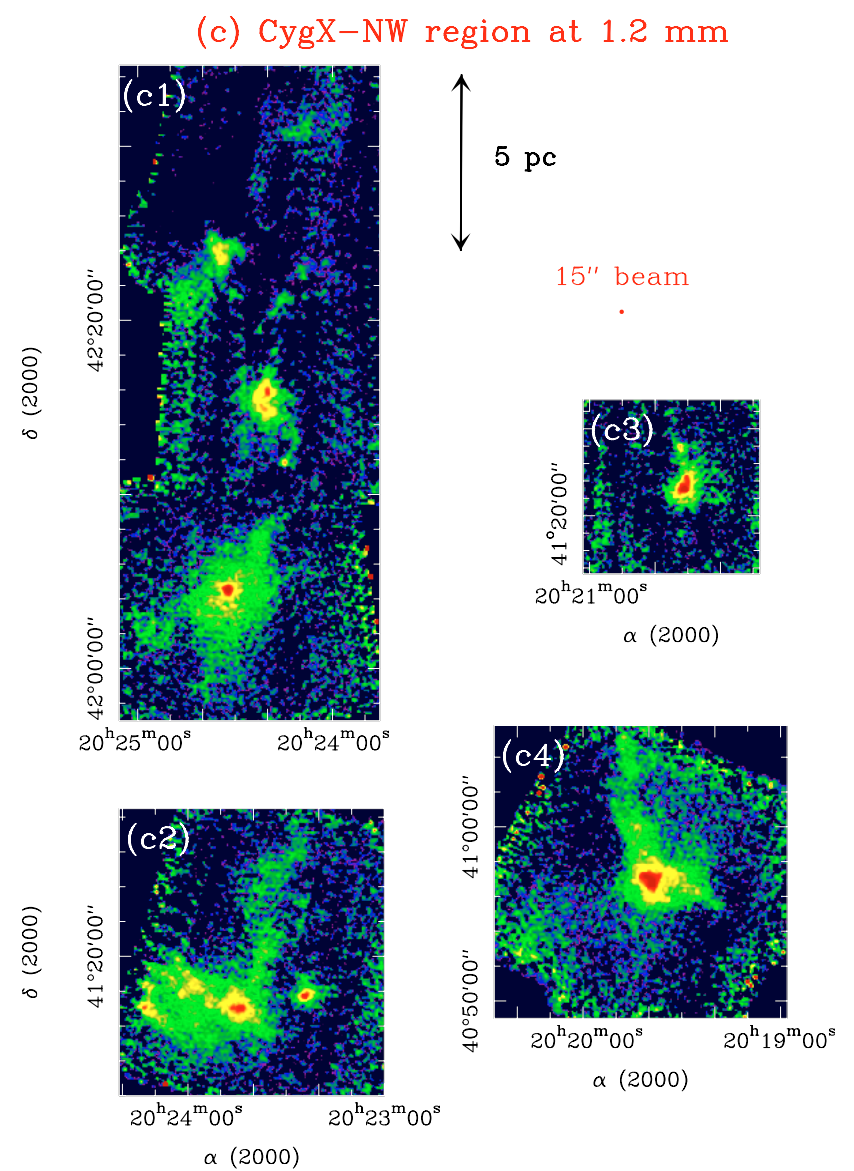

Fig. 2. continued. c) The $\mathrm{CygX}-\mathrm{NW}$ region: maximum flux is $\sim 800 \mathrm{mJy} \mathrm{beam}^{-1}$ (color scale is saturated beyond $500 \mathrm{mJy} \mathrm{beam}^{-1}$ ) and rms noise levels are 9-16 $\mathrm{mJy}_{\text {beam }}{ }^{-1}$ in c1), $\sim 13 \mathrm{mJy} \mathrm{beam}^{-1}$ in c2), $\sim 18 \mathrm{mJy}$ beam $^{-1}$ in $\mathbf{c 3}$ ), and $\sim 12 \mathrm{mJy}_{\text {beam }}{ }^{-1}$ in $\mathbf{c 4}$ ).

peaks of the DR21 filament (cf. Chandler et al. 1993a), as well as the name of nearby benchmark sources (e.g. Carey et al. 2000).

\subsection{Large-scale cloud structures in Cygnus $X$}

To complement the study performed in Sect. 3.2, we here make a census of the large-scale $(\sim 1 \mathrm{pc})$ cloud structures of Cygnus X. We first smooth the MAMBO-2 maps to a 55" resolution, which roughly corresponds to $\sim 0.5 \mathrm{pc}$ and is the resolution of $\mathrm{a} \mathrm{N}_{2} \mathrm{H}^{+}$ survey performed at the FCRAO (Schneider et al. in prep.). We then identify 40 large-scale cloud fragments above the median $5 \sigma=75 \mathrm{mJy}^{-1}$ beam $^{-1}$ level and fit them by a 2-D Gaussian. Note that the skynoise reduction technique we applied may have filtered out part of the large-scale emission (for more details see Appendix A). The present analysis is therefore less secure than that performed in Sect. 3.2 for the small-scale fragments of the Cygnus X molecular clouds. Table 2, which is only available electronically, lists the 40 large-scale clumps found in Cygnus X and gives their $1.2 \mathrm{~mm}$ basic characteristics (in Cols. 1-6) with the same convention as in Table 1. In addition, Col. 7 lists the compact fragments (if any) harbored by each larger-scale structure.

\subsection{Mass and density derived from millimeter continuum maps}

The mass and density of the compact cloud fragments $(<1 \mathrm{pc})$ and large-scale structures $(\sim 1 \mathrm{pc})$ of Cygnus $\mathrm{X}$ are determined from their dust continuum parameters given in Tables 1 and 2 . These tables list the mass (Col. 6 vs. 5) and volume-averaged density (Col. 7 vs. 6) estimated for each identified dense core and clump using methods and assumptions described below.

Before we estimate the mass of each cloud fragment, we should ensure that the millimeter sources identifed in Sects. 3.2 and 3.3 are indeed density structures. According to previous studies of high-mass star forming regions (see e.g. Motte et al. 2003), the main source of contamination of $1.2 \mathrm{~mm}$ fluxes is the free-free emission from $\mathrm{H}$ II regions. Unfortunately, the published centimeter wavelength free-free images of Cygnus X (e.g. Wendker et al. 1991) lack the necessary angular resolution to estimate the amount of likely contamination of each $1.2 \mathrm{~mm}$ source. Furthermore, the spectral index of the free-free emission is unknown, precluding the extrapolation of its flux at $1.2 \mathrm{~mm}$. Observations with high-density tracers such as molecular lines at the IRAM $30 \mathrm{~m}$, Effelsberg, and FCRAO telescopes, as well as $350 \mu \mathrm{m}$ continuum with SHARC II at the CSO, suggest that the vast majority of the $1.2 \mathrm{~mm}$ compact and larger-scale fragments are definitive density structures traced by dust emission rather than free-free centimeter sources (e.g. Schneider et al., in prep.). Fifteen millimeter sources of Table 1 are suspected to contain H II regions (cf. Sect. 5.2.1); their associated masses and densities are therefore upper limits.

We believe that the millimeter continuum emission of cloud fragments identified in Sects. 3.2 and 3.3 is mainly thermal dust emission, which is largely optically thin. For any given dust properties and gas-to-dust ratio, the $1.2 \mathrm{~mm}$ fluxes are thus directly related to the total (gas + dust) mass of the fragments. For present mass estimates, we use the $1.2 \mathrm{~mm}$ integrated fluxes $\left(S_{1.2 \mathrm{~mm}}^{\text {int }}\right)$ of Tables 1,2 without correction for any free-free contamination, and derive the mass $\left(M_{1.2} \mathrm{~mm}\right.$, given in Tables 1,2$)$, as follows:

$$
\begin{aligned}
M_{1.2 \mathrm{~mm}}= & \frac{S_{1.2 \mathrm{~mm}}^{\text {int }} d^{2}}{\kappa_{1.2 \mathrm{~mm}} B_{1.2 \mathrm{~mm}}\left(T_{\text {dust }}\right)} \\
\simeq & 5.3 M_{\odot} \times\left(\frac{S_{1.2 \mathrm{~mm}}^{\text {int }}}{100 \mathrm{mJy}}\right)\left(\frac{d}{1.7 \mathrm{kpc}}\right)^{2} \\
& \times\left(\frac{\kappa_{1.2 \mathrm{~mm}}}{0.01 \mathrm{~cm}^{2} \mathrm{~g}^{-1}}\right)^{-1}\left(\frac{T_{\text {dust }}}{20 \mathrm{~K}}\right)^{-1},
\end{aligned}
$$

where $\kappa_{1.2} \mathrm{~mm}$ is the dust opacity per unit mass column density at $1.2 \mathrm{~mm}, B_{1.2 \mathrm{~mm}}\left(T_{\text {dust }}\right)$ is the Planck function for a dust temperature $T_{\text {dust }}$, and where we assume a distance of $1.7 \mathrm{kpc}$.

The dust mass opacity (including dust properties and gasto-dust mass ratio) is likely to vary with density, temperature, and the evolutionary state of the emitting medium (Henning et al. 1995). Models of dust in low-mass protostellar cores (e.g. Ossenkopf \& Henning 1994) suggest that a value of $\kappa_{1.2 \mathrm{~mm}}=0.01 \mathrm{~cm}^{2} \mathrm{~g}^{-1}$ is well suited for cool $(10-30 \mathrm{~K})$ and high-density $\left(n_{\mathrm{H}_{2}} \sim 10^{5} \mathrm{~cm}^{-3}\right)$ cloud fragments. This value agrees with the cross-comparisons of dust emission surrounding an UCH II region with its $\mathrm{CO}$ ice absorption and gas emission (van der Tak 2002). We therefore choose a dust opacity per unit (gas + dust) mass column density of $\kappa_{1.2 \mathrm{~mm}}=0.01 \mathrm{~cm}^{2} \mathrm{~g}^{-1}$ for compact cloud fragments. We use this value for all the dense fragments because the average dust properties of these $\sim 0.1 \mathrm{pc}$ structures should not change drastically when they contain a 

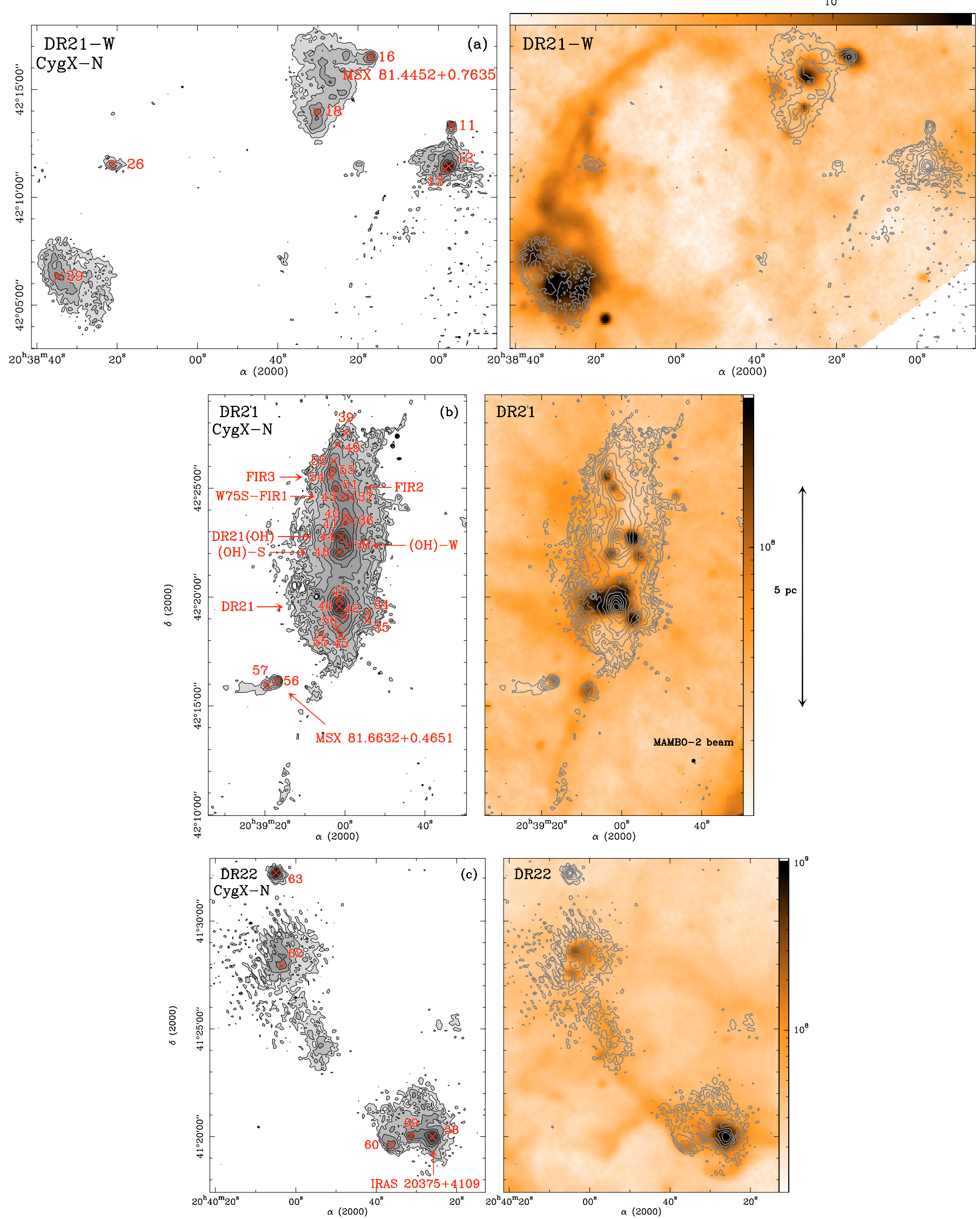

Fig. 3. MAMBO maps of CygX-North (left: gray-scale and contours, right: contours overlaid on $8 \mu \mathrm{m}$ images obtained by $M S X$ and converted to $\mathrm{Jy} \mathrm{s}^{-1}$ ) extracted from Fig. 2a. Regions shown are west of DR21 a), around DR21 b) and DR22 c). The $1.2 \mathrm{~mm}$ and $8 \mu \mathrm{m}$ images have $11^{\prime \prime}$ and $20^{\prime \prime}$ angular resolutions, respectively. The compact cloud fragments discovered in MAMBO images (see Table 1) are labeled and marked by crosses in the gray-scale plot. The infrared sources which coincide with a MAMBO cloud fragment are also indicated. Contour levels are logarithmic and go from 40 to $800 \mathrm{mJy} \mathrm{beam}^{-1}$ in a) and c), and from 40 to $4800 \mathrm{mJy} \mathrm{beam}^{-1}$ in b). 

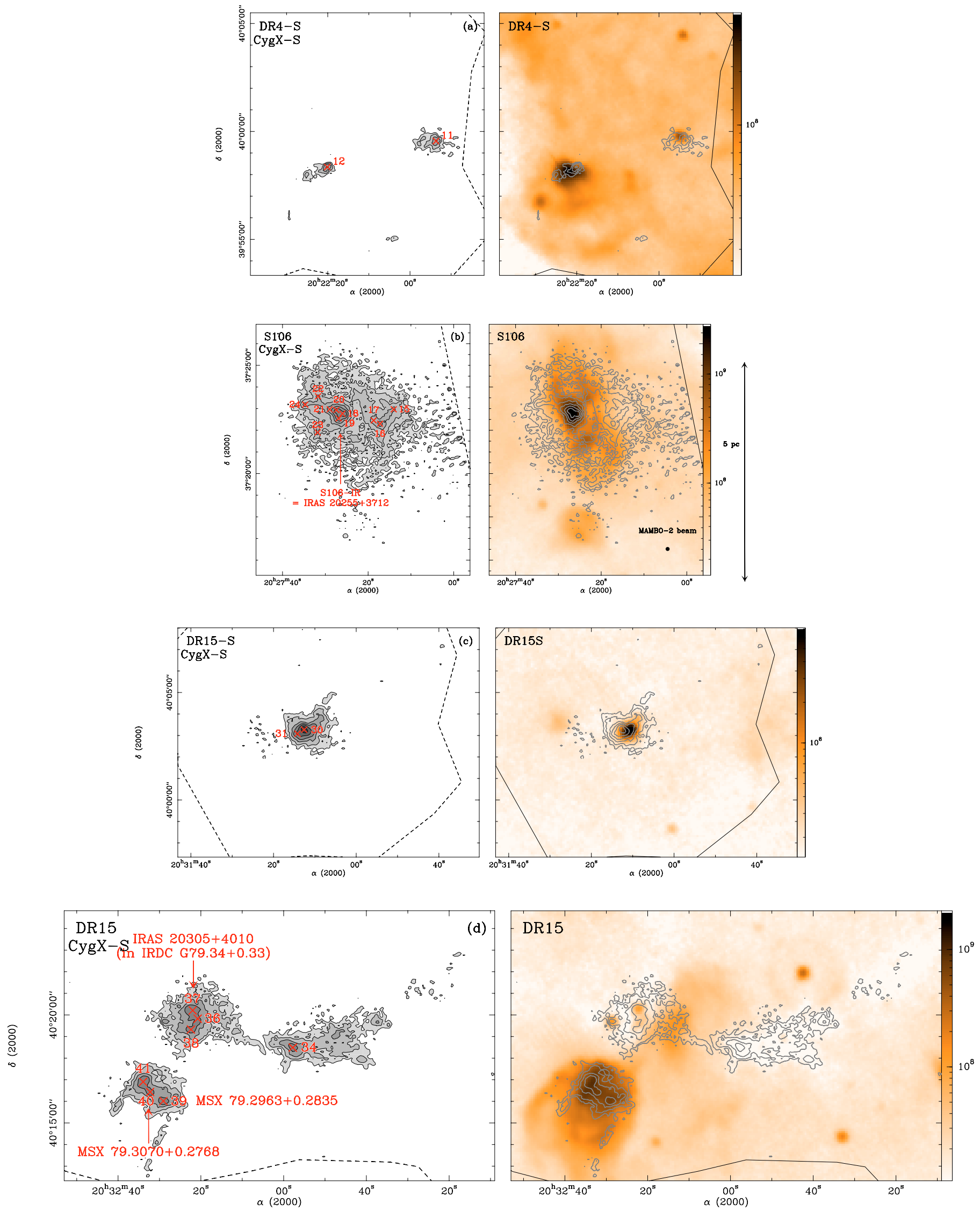

Fig. 4. Same convention as Fig. 3 for MAMBO maps of CygX-South extracted from Fig. 2b. Regions shown are south of DR4 a), around S106 b), south of DR15 c), and around DR15 d). Contour levels are logarithmic and go from 40 to $200 \mathrm{mJy} \mathrm{beam}^{-1}$ in a) and d), from 60 to $1200 \mathrm{mJy}^{-1}$ beam ${ }^{-1}$ in b), and from 40 to $400 \mathrm{mJy}_{\text {beam }}^{-1}$ in c). 
massive protostar. We also adopt $\kappa_{1.2 \mathrm{~mm}}=0.01 \mathrm{~cm}^{2} \mathrm{~g}^{-1}$ for larger-scale $(\sim 1 \mathrm{pc})$ clumps, despite the fact that a lower value is generally used for lower-density starless cloud fragments (e.g. Ward-Thompson et al. 1999). We estimate that the absolute value taken for the dust mass opacity is uncertain by a factor of 2 .

The temperature to be used in Eq. (1) is the mass-weighted dust temperature of the cloud fragments, whose value could be determined from gray-body fitting of their spectral energy distributions. Such measures are not yet available but we recently measured the ammonia rotational temperatures of the Cygnus $\mathrm{X}$ dense cores (Wyrowski et al. in prep.). The ratios of $\mathrm{NH}_{3}(1,1)$ and $(2,2)$ transitions observed with the Effelsberg $100 \mathrm{~m}$ telescope $\left(40^{\prime \prime}\right.$ beam) give cold temperatures $(\sim 15 \mathrm{~K})$ for the material located within a $0.33 \mathrm{pc}$ diameter of a millimeter peak. To estimate the temperature averaged on $\sim 0.1 \mathrm{pc}$ scales, we assume a classical $\rho(r) \propto r^{-2}$ density law and inner heating $\left(T(r) \propto r^{-0.4}\right)$, in agreement with small-scale fragments being highly centrally condensed and generally protostellar (cf. Sects. 5.1, 5.2). We thus estimate that $\sim 0.1 \mathrm{pc}$ fragments should have mass-weighted dust temperatures in the range of $15-25 \mathrm{~K}$. This does not preclude any hotter or colder components from being embedded in the $\sim 0.1$ pc fragments; temperature gradients will be discussed in companion papers (Schneider et al. in prep.; Wyrowski et al. in prep.). For simplicity, we assume $T_{\text {dust }}=20 \mathrm{~K}$ in Eq. (1) for all the cloud fragments of Table 1 . We add estimates made with $T_{\text {dust }}=40 \mathrm{~K}$ for $\mathrm{H}$ II regions (identified in Sect. 5.2.1) and the brightest $\left(L_{\text {bol }}>5 \times 10^{3} L_{\odot}\right)$ infrared protostars of Cygnus X: IRAS 20343+4129, studied by Beuther et al. (2002) which corresponds to CygX-N5, and LkHA 225S which coincides with CygX-NW5. To avoid biasing our statistical results, we hereafter use the estimates made with a homogeneous $20 \mathrm{~K}$ temperature. The dust temperature of large-scale clumps is more difficult to constrain since it may vary according to the presence or absence of strong external heating. We estimate that the massweighted dust temperature may vary from $T_{\text {dust }}=10 \mathrm{~K}$ to $20 \mathrm{~K}$, and choose $T_{\text {dust }}=15 \mathrm{~K}$ as an averaged value to use in Eq. (1) for the large-scale $(\sim 1 \mathrm{pc})$ clumps of Table 2 .

The mass estimates in Tables 1,2 are globally uncertain by a factor of 2 due to uncertain dust emissivity. The individual masses can vary by $\pm 30 \%$ relative to each other when dust temperatures vary from 15 to $25 \mathrm{~K}$, or by $\pm 50 \%$ for the 10 to $20 \mathrm{~K}$ temperature range (see also Eq. (1)).

The volume-averaged densities listed in Tables 1,2 and used in Tables 4, 5 are estimated as follows:

$\left\langle n_{\mathrm{H}_{2}}\right\rangle=\frac{M_{1.2 \mathrm{~mm}}}{\frac{4}{3} \pi \times F W H M^{3}}$,

where $M_{1.2} \mathrm{~mm}$ is the mass derived by Eq. (1) and FWHM is the full width at half maximum, determined by Gaussian fits. Using a radius equal to one $F W H M$ in Eq. (2) allows one to accurately determine the volume-averaged density because the flux (and thus the mass) measured within such an aperture corresponds to $>98 \%$ of the integrated flux (respectively total mass) of Gaussian cloud structures. The often-used, beam-averaged peak density would of course be higher, but is less relevant when estimating physical constraints such as the free-fall time.

\section{Signpost of stellar activity toward the Cygnus $X$ cloud fragments}

We investigate the spatial coincidence of the compact cloud fragments identified in Sect. 3.2 with mid-infrared, SiO, centimeter free-free, and maser emission (see Sects. 4.1-4.3). Large-scale cloud structures identified in Sect. 3.3, which are associated with neither compact fragments displaying signposts of stellar activity nor bright mid-infrared sources, qualify as starless clumps as indicated in Col. 7 of Table 2.

\subsection{Coincidence with mid-infrared point sources}

When searching for the signature of embedded stellar embryos, the cross-correlation of compact millimeter sources with infrared catalogs is crucial. Among the near- to mid-infrared surveys that are currently available and complete for the Cygnus $\mathrm{X}$ complex, we choose that performed by the $M S X^{3}$ satellite; its angular resolution $\left(20^{\prime \prime}\right)$ is far better than that provided by IRAS and its wavebands (from 8 to $21 \mu \mathrm{m}$ ) are better suited than those of 2MASS. The Spitzer satellite provides better angular resolution and sensitivity but the mapping of Cygnus $\mathrm{X}$ with the Spitzer/MIPS camera is far from being complete, and published results from the Spitzer/IRAC camera generally do not crosscorrelate well with (sub)millimeter sources (see Davis et al. 2007; and the counterexample ERO1/CygX-N57 of Marston et al. 2004).

To search for coincidence with mid-infrared point sources, we carefully examine the structure of the $M S X$ emission at the exact location of each compact millimeter source (see right-hand side of Figs. 3, 4 and Figs. 12-14). A millimeter source is defined to be detected by $M S X$ if it coincides with pointlike emission at both 8 and $21 \mu \mathrm{m}$. Slightly more extended $M S X$ emission is considered for three developed H II regions (cf. Sect. 5.2.1): DR21 (associated with CygX-N46 and N47), S106-IR (associated with CygX-S18, S19 and S20) and IRAS 20306+4005 (associated with CygX-S39 and S40). Given the resolution of $M S X$ images, $M S X$ point sources have similar sizes to those of compact cloud fragments detected by MAMBO-2. The detection, at both 8 and $21 \mu \mathrm{m}$, allows the rejection of peaks of infrared emission created by small grains at the interface of photodissociation regions and molecular clumps (e.g. Abergel et al. 2002). The latter are actually externally heated sources whose peaks shift with wavelength and sometimes disappear at $21 \mu \mathrm{m}$. We add four more sources which are only detected at $21 \mu \mathrm{m}$ : CygX-N30 (W75N(B)), CygX-N46 (DR21) and N47 (DR21-D), whose $8 \mu \mathrm{m}$ emission is extinguished by their high-column density, and CygX-N65, which is very weak. The positional accuracy requested between the compact sources of MAMBO-2 and $M S X$ is $10^{\prime \prime}$ (i.e. the sum of the maximum pointing errors of both images), which corresponds to $0.08 \mathrm{pc}$ at $1.7 \mathrm{kpc}$. Such a narrow association is made possible by the small size $(\sim 0.1 \mathrm{pc})$ of the compact cloud fragments extracted from the MAMBO-2 images. In fact if we consider looser associations, many $M S X$ point sources lie on and possibly within clumps, at $\sim 0.2$ pc from the density peaks which we identified as compact fragments (e.g. Figs. 3a, b). We reject these loose associations, unlike previous studies that considered, for example, that CygX-S12 (respectively CygX-S30) and IRAS 20205+3948 (respectively IRAS 20293+3952) coincide (cf. Figs. 4a and c; see also Beuther et al. 2002). In fact, these MSX point sources lying within dust clumps sometimes coincide with extremely red objects detected by Spitzer/IRAC (EROs, Marston et al. 2004; Davis et al. 2007), confirming that (sub)millimeter and infrared surveys generally identify different population of stars in terms of age and/or mass.

\footnotetext{
3 The Midcourse Space Experiment is a Department of Defense program that provides images observed at $8,12,15$, and $21 \mu \mathrm{m}$ with a $20^{\prime \prime}$ resolution.
} 
Our cross-correlations are illustrated in the right-hand parts of Figs. 3, 4 and Figs. 12-14. Their results are given in Col. 8 of Table 1 . When there is a coincidence with a $M S X$ point source, Col. 8 gives its $21 \mu \mathrm{m}$ flux as taken from the $M S X$ catalog (see Note $\mathrm{c}$ for exceptions) and Col. 9 gives the IRAS or MSX name. Otherwise, when the MAMBO-2 source is not detected or seen in absorption at $8 \mu \mathrm{m}$, Col. 8 of Table 1 mentions "-" or "Abs".

With the above selection criteria, 36 compact cloud fragments of Cygnus X (i.e. less than $1 / 3$ of the sample) are associated with mid-infrared emission detected by $M S X$. The other 93 fragments are undetected down to $\sim 0.15 \mathrm{Jy}$ at $8 \mu \mathrm{m}$ and $\sim 3 \mathrm{Jy}$ at $21 \mu \mathrm{m}$, which are the completeness levels of the MSX catalog (MSX C6) in Cygnus X (see Egan et al. 2003; Bontemps et al. in prep.). Some of these sources are even seen in absorption against the diffuse mid-infrared background. Interestingly, some of the Cygnus X fragments which are undetected by MSX harbor a weak $24 \mu \mathrm{m}$ source in Spitzer images. To preserve the homogeneity of our study, we do not use this information here.

\subsection{Survey for SiO emission}

Silicon monoxide ( $\mathrm{SiO}$ ) emission is an excellent tracer of shocked gas, usually associated with molecular outflows (e.g. Ziurys \& Friberg 1987; Martín-Pintado et al. 1992; Schilke et al. 1997). SiO may also have been detected in shocks toward hot cores (e.g. Hatchell et al. 2001, and references therein). We have surveyed for $\mathrm{SiO}(2-1)$ emission by making single pointings toward almost ${ }^{4}$ all the most massive $\left(M_{1.2} \mathrm{~mm} \geq 40 M_{\odot}\right)$ fragments of Table 1 and a few less massive fragments. Figures 5 and 15 (only provided electronically) display the $\mathrm{SiO}(2-1)$ lines for all the observed fragments, ordered by decreasing $M_{1.2} \mathrm{~mm}$. In Table 3 and for each $1.2 \mathrm{~mm}$ compact fragment (Col. 1), we give the peak line temperature (Col. 3), the line width at half maximum (Col. 4), the full line width at the base (Col. 5), and the integrated intensity (Col. 6) of the $\mathrm{SiO}(2-1)$ emission. For direct comparison, we list the $\mathrm{SiO}$ line parameters scaled to the Cygnus $\mathrm{X}$ distance of three nearby protostars which are among the brightest known $\mathrm{SiO}$ outflow sources.

Among the 28 most massive fragments whose $\mathrm{SiO}$ emission is displayed in Fig. 5, only two (CygX-S18, and CygX-N6) are not detected. This implies a very high detection rate of $93 \%$ ( $>79 \%$ when considering the five high-mass fragments which have not been observed), with a typical detection level of $\sim 0.15 \mathrm{~K} \mathrm{~km} \mathrm{~s}^{-1}$ (see Table 3). This detection rate stays high even when considering the observation of the 20-40 $M_{\odot}$ dense cores listed in Table 3 . Down to $\sim 25 M_{\odot}$, the compact fragments of Table 3 that do not have any SiO emission are all associated with infrared point sources: CygX-S18/S106-IR, CygXN6/IRAS 20343+4129, and CygX-N16/MSX 81.4452+0.7635. This suggests that all massive fragments down to at least $40 M_{\odot}$, and maybe $25 M_{\odot}$, drive $\mathrm{SiO}$ outflows and/or contain hot cores.

In Fig. 5, we show the emission spectra separately for the high-luminosity infrared sources $\left(\geq 10^{3} L_{\odot}\right)$ and the massive infrared-quiet cores (cf. their definitions given in Sects. 5.2.2 and 5.3.1). The two groups obviously have different behavior. All infrared-quiet cores are detected in $\mathrm{SiO}(2-1)$ and most of them are particulary bright; the averaged peak line temperature and integrated intensity are $0.55 \mathrm{~K}$ and $4.6 \mathrm{~K} \mathrm{~km} \mathrm{~s}^{-1}$, respectively. In contrast, only a small fraction of the high-luminosity

\footnotetext{
4 Three sources (CygX-N32, N47, and S20) are included in beams targeting other fragments (CygX-N30, N46, and S18 respectively). Two sources (CygX-N69 and CygX-S10) were not observed because they are among the lowest-density fragments.
}

infrared sources show a strong $\mathrm{SiO}$ line; the averaged peak line temperature and integrated intensity are $0.22 \mathrm{~K}$ and $1.9 \mathrm{~K} \mathrm{~km} \mathrm{~s}^{-1}$, respectively. Since only two high-luminosity infrared sources (CygX-N48/DR21 and CygX-N30/W75N) are strong SiO emitters, the contrast is even larger when comparing the median values: $0.7 \mathrm{~K} \mathrm{~km} \mathrm{~s}^{-1}$ for the high-luminosity infrared sources, and $3.1 \mathrm{~K} \mathrm{~km} \mathrm{~s}^{-1}$ (i.e. 4.3 times more) for the infrared-quiet cores. The $\mathrm{SiO}$ lines of infrared-quiet cores are also 3 to 4 times brighter than the brightest $\mathrm{SiO}$ outflows of the nearby low-mass protostars (see Table 3 ).

Almost all the SiO lines shown in Figs. 5 and 15 display outflow wings with line width at the base of emission that can reach up to $\sim 60 \mathrm{~km} \mathrm{~s}^{-1}$. Among the ten brightest $\mathrm{SiO}$ sources, the average of the line width at the base is as high as $41 \mathrm{~km} \mathrm{~s}^{-1}$, thus clearly suggesting outflow shocks. A few $\mathrm{SiO}$ lines might also indicate the presence of at least two emission components: a wide base (due to outflow shocks) and a narrower component which may indicate a contribution of shocks inside a hot core and/or lower velocity outflows. The best examples of two-component lines are those detected toward CygX-S26/AFGL 259, CygX-N44/DR21(OH), and CygX-N48/DR21(OH)-S, while the best example of singlecomponent lines suggestive of a unique powerful outflow are CygX-N46/DR21, CygX-N53, CygX-S30, and CygX-N68.

\subsection{Coincidence with radio free-free and maser emission}

We have used the SIMBAD ${ }^{5}$ database to search for complementary signposts of stellar activity: centimeter free-free emission and $\mathrm{OH}, \mathrm{H}_{2} \mathrm{O}$, and $\mathrm{CH}_{3} \mathrm{OH}$ masers. SIMBAD gives inhomogeneous information on our sample of 129 compact cloud fragments, since it compiles results of Cygnus X surveys performed with limited angular resolution and high-angular resolution studies made for well-known sources.

Within Table 1, fifteen dense cores coincide with a smalldiameter $\left(\leq 10^{\prime \prime}\right)$ source of radio emission detected at 2, 3.6, 6, 11, or $21 \mathrm{~cm}$ (e.g. Kurtz et al. 1994; Zoonematkermani et al. 1990). We cannot check the nature of the detected centimeter emissions with the current database, but it most likely corresponds to free-free emissions emitted by $\mathrm{H}$ II regions. Furthermore, several $1.2 \mathrm{~mm}$ continuum sources coincide with $\mathrm{OH}, \mathrm{H}_{2} \mathrm{O}$, and/or $\mathrm{CH}_{3} \mathrm{OH}$ maser sources (e.g. Braz \& Epchtein 1983; Valdettaro et al. 2001; Pestalozzi et al. 2005). Most of these dense cores are associated with centimeter continuum and/or mid-infrared emission, but one (CygX-N53) is neither a centimeter nor a MSX source. In Col. 9 of Table 1, we indicate when some centimeter free-free and/or maser emission is detected toward a Cygnus X dense core.

\section{Discussion}

\subsection{A sample of high-density cores}

Our $1.2 \mathrm{~mm}$ continuum imaging of the Cygnus $\mathrm{X}$ molecular cloud complex gives a complete view of the structure of its highest-column density portions, with spatial scales ranging from $0.03 \mathrm{pc}$ (deconvolved from the beam) to $3 \mathrm{pc}$. According to the terminology used for nearby molecular clouds (e.g. Williams et al. 2000), it covers scales typical for dense cores ( $\sim 0.1 \mathrm{pc})$ and clumps $(\sim 1 \mathrm{pc})$. The 129 compact cloud fragments identified in

\footnotetext{
5 SIMBAD is the reference database for identification and bibliography of astronomical objects, developed and maintained by CDS, Strasbourg.
} 

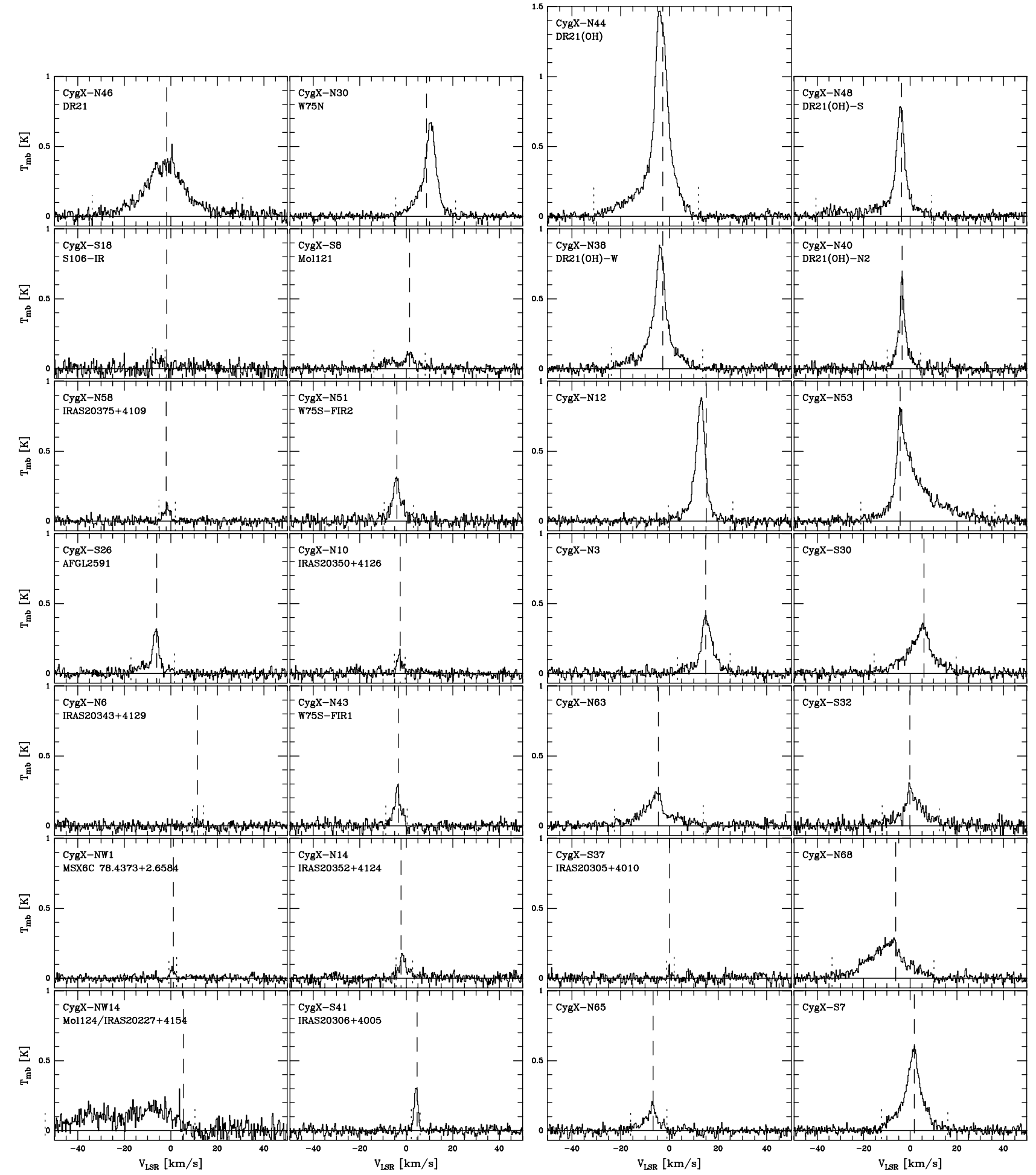

Fig. 5. $\mathrm{SiO}(2-1)$ lines observed toward the 28 most massive $\left(\geq 40 M_{\odot}\right)$ cloud fragments of Cygnus X: 14 high-luminosity infrared sources on the left and 14 infrared-quiet protostellar cores on the right, both ordered by decreasing $M_{1.2} \mathrm{~mm}$ from top to bottom. The local velocity at rest of each cloud fragment, as measured by optically-thin tracers, is indicated by a dashed line.

Cygnus $\mathrm{X}$ have sizes ranging from 0.03 to $0.3 \mathrm{pc}$ with a mean size of $\sim 0.1 \mathrm{pc}$ (see Sect. 3.2 and Table 1). They should therefore be called "dense cores". The 40 larger-scale structures of Table 2 have typical sizes of $\sim 0.7 \mathrm{pc}$; we will call them "clumps". In Table 4, we give the mean properties of the Cygnus $\mathrm{X}$ dense cores and clumps and compare them with those of a few representative studies (Motte et al. 1998; Ward-Thompson et al. 1999; Beuther et al. 2002; Rathborne et al. 2006). In Fig. 6a, b, we plot the mass and density distributions of these $1.2 \mathrm{~mm}$ sources samples.

The typical size of the Cygnus $\mathrm{X}$ dense cores is 5 to 10 times smaller than the average cloud structures identified by 
Table 3. $\mathrm{SiO}(2-1)$ observations of the most massive dense cores of Cygnus X compared with the brightest $\mathrm{SiO}$ protostellar sources at $<500$ pc.

\begin{tabular}{|c|c|c|c|c|c|c|}
\hline $\begin{array}{l}\text { Source } \\
\text { name }\end{array}$ & $\begin{array}{l}M_{1.2 \mathrm{~mm}} \\
\quad\left(M_{\odot}\right)\end{array}$ & $\begin{array}{l}T_{\mathrm{SiO}} \\
(\mathrm{K})\end{array}$ & $\begin{array}{l}\Delta v_{F W H M} \\
\left(\mathrm{~km} \mathrm{~s}^{-1}\right)\end{array}$ & $\begin{array}{l}\Delta v_{\text {outflow }} \\
\left(\mathrm{km} \mathrm{s}^{-1}\right)\end{array}$ & $\underset{\left(\mathrm{K} \mathrm{km} \mathrm{s}^{-1}\right)}{T_{\mathrm{SiO}} \mathrm{d} v}$ & Possible nature \\
\hline N46, DR21 & $<609-949$ & 0.38 & 16.9 & 64.8 & $8.6 \pm 0.42$ & UCH II region \\
\hline $\mathrm{N} 30, \mathrm{~W} 75 \mathrm{~N}(\mathrm{~B})$ & $<361-563$ & 0.65 & 5.4 & 25.8 & $4.7 \pm 0.16$ & hyper compact H II region? \\
\hline $\mathrm{N} 44, \mathrm{DR} 21(\mathrm{OH})$ & 446 & 1.45 & 6.0 & 43.0 & $13.4 \pm 0.25$ & massive IR-quiet protostellar core \\
\hline S18, S106-IR & $<145-226$ & $<0.10$ & - & - & $<0.15$ & compact $\mathrm{H}$ II region \\
\hline $\mathrm{N} 48, \mathrm{DR} 21(\mathrm{OH})-\mathrm{S}$ & 197 & 0.78 & 4.0 & 49.7 & $5.4 \pm 0.26$ & massive IR-quiet protostellar core \\
\hline S8, Mol 121S & $<121-188$ & 0.10 & 4.6 & 22.1 & $0.96 \pm 0.18$ & UCH II region \\
\hline N58, IRAS $20375+4109$ & $<92-144$ & 0.086 & 3.8 & 7.1 & $0.34 \pm 0.10$ & compact $\mathrm{H}$ II region \\
\hline N38, DR21(OH)-W & 138 & 0.86 & 4.6 & 37.5 & $6.8 \pm 0.25$ & massive IR-quiet protostellar core \\
\hline N51, W75S-FIR2 & 125 & 0.29 & 3.6 & 12.7 & $1.5 \pm 0.19$ & high-luminosity IR protostellar core \\
\hline S26, AFGL2591 & $<80-124$ & 0.30 & 2.8 & 18.7 & $1.5 \pm 0.19$ & UCH II region \\
\hline $\mathrm{N} 40, \mathrm{DR} 21(\mathrm{OH})-\mathrm{N} 2$ & 106 & 0.63 & 2.6 & 14.4 & $2.3 \pm 0.18$ & massive IR-quiet protostellar core \\
\hline N12 & 86 & 0.87 & 4.0 & 26.5 & $4.7 \pm 0.21$ & massive IR-quiet protostellar core \\
\hline N53 & 85 & 0.80 & 6.4 & 57.6 & $8.9 \pm 0.31$ & massive IR-quiet protostellar core \\
\hline N3 & 84 & 0.40 & 4.8 & 21.5 & $2.6 \pm 0.21$ & massive IR-quiet protostellar core \\
\hline S30 & 82 & 0.32 & 9.3 & 35.2 & $3.9 \pm 0.26$ & massive IR-quiet protostellar core \\
\hline N10, IRAS $20350+4126$ & $<52-81$ & 0.12 & 2.4 & 4.7 & $0.35 \pm 0.10$ & UCH II region? \\
\hline N6, IRAS 20343+4129 & $44-68$ & $<0.07$ & - & - & $<0.10$ & high-luminosity IR protostellar core \\
\hline N43, W75S-FIR1 & 66 & 0.25 & 3.4 & 9.1 & $0.94 \pm 0.15$ & high-luminosity IR protostellar core \\
\hline NW1, IRAS20178+4046 & $<38-59$ & 0.05 & 2.6 & 3.4 & $0.15 \pm 0.06$ & UCH II region? \\
\hline N63 & 58 & 0.23 & 7.2 & 36.5 & $2.6 \pm 0.23$ & massive IR-quiet protostellar core \\
\hline S32 & 54 & 0.29 & 6.2 & 24.6 & $2.4 \pm 0.28$ & massive IR-quiet protostellar core \\
\hline N14, IRAS $20352+4124$ & 50 & 0.16 & 2.4 & 7.1 & $0.61 \pm 0.13$ & high-luminosity IR protostellar core \\
\hline NW14, Mol124 & $32-50$ & 0.16 & 41.6 & 64.4 & $5.8 \pm 0.76$ & high-luminosity IR protostellar core \\
\hline S37, IRAS $20305+4010$ & 45 & 0.05 & - & 3.2 & $0.09 \pm 0.09$ & massive IR-quiet protostellar core \\
\hline N68 & 44 & 0.27 & 13.7 & 43.8 & $4.6 \pm 0.25$ & massive IR-quiet protostellar core \\
\hline S41, IRAS $20306+4005$ & $27-42$ & 0.30 & 2.0 & 8.2 & $0.61 \pm 0.09$ & high-luminosity IR protostellar core \\
\hline N65 & 41 & 0.19 & 3.8 & 16.9 & $1.06 \pm 0.16$ & massive IR-quiet protostellar core \\
\hline S7 & 40 & 0.58 & 6.0 & 28.4 & $5.1 \pm 0.23$ & massive IR-quiet protostellar core \\
\hline NW2 & 36 & 0.10 & - & 2.0 & $0.18 \pm 0.09$ & IR-quiet protostellar core \\
\hline S43 & 35 & 0.16 & 2.2 & 6.1 & $0.59 \pm 0.13$ & IR-quiet protostellar core \\
\hline N24 & 34 & 0.09 & 4.7 & 5.1 & $0.39 \pm 0.10$ & IR-quiet protostellar core \\
\hline NW5, LkHA 225S & $22-34$ & 0.045 & - & 14.9 & $0.69 \pm 0.26$ & infrared protostellar core \\
\hline N60 & 30 & 0.08 & 2.0 & 4.5 & $0.20 \pm 0.10$ & IR-quiet protostellar core \\
\hline N16, MSX 81.4452+0.7635 & 29 & $<0.09$ & - & - & $<0.12$ & infrared protostellar core \\
\hline N28 & 27 & 0.09 & 1.8 & 3.5 & $0.25 \pm 0.09$ & IR-quiet protostellar core \\
\hline N62 & 27 & 0.10 & 2.6 & 8.5 & $0.42 \pm 0.11$ & IR-quiet protostellar core \\
\hline S16 & 24 & 0.10 & 2.6 & 14.5 & $0.51 \pm 0.13$ & IR-quiet protostellar core \\
\hline N18 & 23 & $<0.08$ & - & - & $<0.11$ & ? \\
\hline N59 & 20 & $<0.08$ & - & - & $<0.11$ & ? \\
\hline NW9, IRAS 20216+4107 & 19 & $<0.10$ & - & - & $<0.15$ & infrared protostellar core \\
\hline L1448-MM @ 1.7 kpc & - & - & - & $\sim 140$ & $\sim 1.4^{a}$ & low-mass class 0 protostar \\
\hline L1157-MM @1.7 kpc & - & - & - & $\sim 25$ & $\sim 1.8^{b}$ & low-mass class 0 protostar \\
\hline Orion-IRc2@1.7 kpc & - & - & - & $\sim 74$ & $\sim 9.2^{c}$ & high-mass protostar \\
\hline
\end{tabular}

${ }^{a}$ Intensity which has been distance-corrected (extrapolation from $300 \mathrm{pc}$ to $1.7 \mathrm{kpc}$ ) using values from Table 2 of Nisini et al. (2007) and excluding R4 which would fall outside the beam.

${ }^{b}$ Intensity which has been distance-corrected (extrapolation from $440 \mathrm{pc}$ to $1.7 \mathrm{kpc}$ ) using values from Table 3 of Nisini et al. (2007) and summing up $\mathrm{B} 0$ and $\mathrm{B} 1$ components.

${ }^{c}$ Intensity which has been distance-corrected (extrapolation from $450 \mathrm{pc}$ to $1.7 \mathrm{kpc}$ ) using the spectrum of Fig. 1 in Ziurys \& Friberg (1987).

single-dish studies of high-mass star-forming regions (see Table 4; and also Reid \& Wilson 2005; Hill et al. 2005). Using the terminology mentioned above, HMPOs (for high-mass protostellar objects, Beuther et al. 2002) and IRDCs (for infrared dark clouds, e.g. Rathborne et al. 2006) are actually "clumps". We are here able to reach the scale of dense cores because Cygnus $\mathrm{X}$ is one of the closest high-mass star-forming regions, our MAMBO-2 survey has a good spatial resolution, and we deliberately focus on high-density cloud structures. As a consequence of their small sizes, the Cygnus $\mathrm{X}$ dense cores have masses (4-950 $M_{\odot}$, see Table 1) which are up to one order of magnitude smaller than those obtained by Beuther et al. (2002) and Rathborne et al. (2006) (see Table 4 and Fig. 6a). In contrast, Cygnus X dense cores have densities averaged over their FWHM size $\left(1 \times 10^{4}-2 \times 10^{6} \mathrm{~cm}^{-3}\right)$ which are more than one order of magnitude higher than those of HMPOs and IRDCs (cf. Table 4 and Fig. 6b). As interesting illustrations, the Cygnus X HMPOs called IRAS 20343+4129 and IRAS 20293+3952 (Beuther et al. 2002) are twice as large and 7 times less dense than the dense cores we have identified within them (see CygX-N5, CygX-N6 and CygX-S30 in Table 1).

On average, the Cygnus $\mathrm{X}$ dense cores are a factor of 5 more massive and a factor of 3 denser than nearby dense cores identified by Motte et al. (1998) and Ward-Thompson et al. (1999) (see Table 4 and Figs. 6a, b). The latter sample includes isolated pre-stellar cores located at $140-440 \mathrm{pc}$ from the Sun, and all the protocluster dense cores of the $\rho$ Ophiuchi main cloud which are either pre-stellar or already forming low-mass stars. Statistically, 
Table 4. Mean properties of the Cygnus X clumps and cores compared with millimeter cloud structures of a few reference studies.

\begin{tabular}{|c|c|c|c|c|c|c|c|}
\hline & $\begin{array}{l}\text { HMPOs } \\
\text { clumps }\end{array}$ & $\begin{array}{l}\text { IRDCs } \\
\text { clumps }\end{array}$ & $\begin{array}{l}\text { CygX clumps } \\
\text { (all / starless) }\end{array}$ & $\begin{array}{l}\text { Cygnus X } \\
\text { dense cores }\end{array}$ & $\begin{array}{c}\geq 40 M_{\odot} \mathrm{CygX} \\
\text { dense cores }\end{array}$ & $\begin{array}{l}\text { Nearby, low-mass } \\
\text { dense cores }\end{array}$ & $\begin{array}{c}\rho \mathrm{Oph} \\
\text { condensations }\end{array}$ \\
\hline$F W H M^{a}$ size $(\mathrm{pc})$ & 0.5 & 0.5 & $0.68 / 0.78$ & 0.10 & 0.13 & 0.08 & 0.007 \\
\hline $\operatorname{Mass}^{b} M_{12} \mathrm{~mm}\left(M_{\odot}\right)$ & 290 & 150 & $1000 / 780$ & 24 & 91 & 4.7 & 0.15 \\
\hline$\left\langle n_{\mathrm{H}_{2}}\right\rangle^{c}\left(\mathrm{~cm}^{-3}\right)$ & $8.5 \times 10^{3}$ & $5.9 \times 10^{3}$ & $1.4 \times 10^{4} / 6.7 \times 10^{3}$ & $1.1 \times 10^{5}$ & $1.9 \times 10^{5}$ & $3.5 \times 10^{4}$ & $1.9 \times 10^{6}$ \\
\hline Number of sources & $69^{e}$ & 190 & $40 / 10$ & 128 & 33 & 22 & 60 \\
\hline References $^{d}$ & (1) & (2) & (3) & (3) & (3) & $(4),(5)$ & (5) \\
\hline Distance $(\mathrm{kpc})$ & $0.3-14$ & $1.8-7.1$ & 1.7 & 1.7 & 1.7 & $0.14-0.44$ & 0.14 \\
\hline
\end{tabular}

${ }^{a}$ Deconvolved FWHM sizes derived from a 2D-Gaussian fit, except for HMPOs and IRDCs for which sizes are taken from Table 1 of Beuther et al. (2002) (mean estimates are taken when the distance is unknown) and Table 3 of Rathborne et al. (2006).

${ }^{b}$ Mass consistently estimated from the $1.2 \mathrm{~mm}$ integrated flux using Eq. (1). The mass values of Beuther et al. (2002) have been recalculated using $\kappa_{1.2 \mathrm{~mm}}=0.01 \mathrm{~cm}^{2} \mathrm{~g}^{-1}$ but those of nearby, low-mass dense cores and $\rho$ Oph condensations are kept while they assume $\kappa_{1.2 \mathrm{~mm}}=0.005 \mathrm{~cm}^{2} \mathrm{~g}^{-1}$.

$c$ Volume-averaged density homogeneously recalculated using Eq. (2) for every list of sources.

${ }^{d}$ References: (1) Beuther et al. (2002); (2) Rathborne et al. (2006); (3) this paper; (4) Ward-Thompson et al. (1999); (5) Motte et al. (1998).

${ }^{e}$ We limit our comparison to the main HMPOs components, which are the most likely progenitors of high-mass stars.
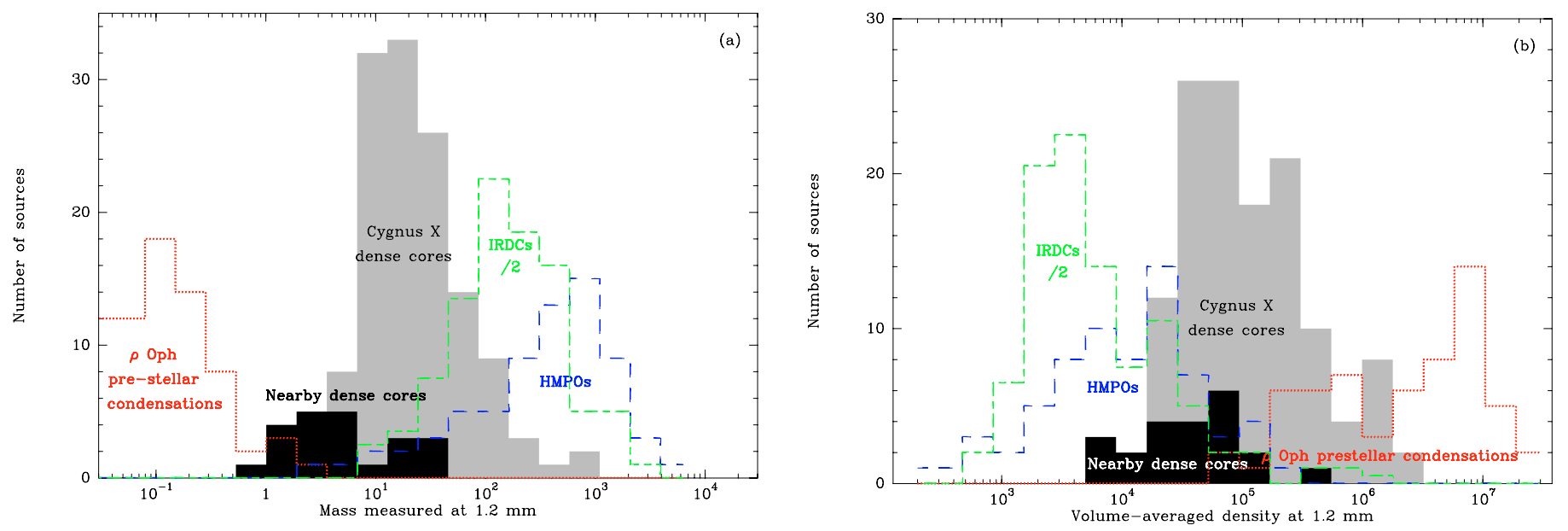

Fig. 6. Distribution of mass a) and volume-averaged density b) of the Cygnus X dense cores compared to those of clumps hosting high-luminosity protostars (main component of HMPOs, Beuther et al. 2002) and clumps referred to as IRDCs (Rathborne et al. 2006). The Cygnus X histograms are also compared to density spectra of nearby dense cores (Motte et al. 1998; Ward-Thompson et al. 1999) and $\rho$ Oph condensations (Motte et al. 1998).

studying the Cygnus $\mathrm{X}$ region gives access to dense cores which are one to two orders of magnitude more massive than those observed in nearby regions. The 33 most massive compact cloud fragments of Cygnus X (i.e. with masses larger than $40 M_{\odot}$, see Table 3) already have 19 times more mass than the dense cores of low-mass star-forming regions (cf. Table 4). Thus, they do not have any equivalent in the nearby star-forming regions and represent good candidate sites for forming intermediate- to highmass stars.

In terms of average density, Cygnus $\mathrm{X}$ dense cores are intermediary cloud structures between nearby dense cores and condensations in the $\rho$ Ophiuchi main cloud (cf. Table 4 and Fig. 6b). This result suggests that the efficiency of mass transfer from the gas reservoir to the star(s) is high. Indeed, the star formation efficiency estimated in the $\rho$ Ophiuchi dense cores is $\sim 31 \%$ over $10^{6}$ yr (Bontemps et al. 2001), and the good agreement in $\rho$ Oph between the pre-stellar condensation mass spectrum and the stellar IMF suggests an efficiency of 50-100\% (Motte et al. 1998; Bontemps et al. 2001). While the low-mass starless condensations are believed to be the direct progenitors of single stars, the Cygnus $\mathrm{X}$ dense cores will probably form small groups of stars. The large mass of the Cygnus $\mathrm{X}$ dense cores, compared to that of nearby dense cores and $\rho$ Oph condensations, argues for the formation of intermediate- to high-mass stars (cf. Fig. 6a). With their high density and small size, the Cygnus $\mathrm{X}$ dense cores can probably be seen as the inner part of clumps/protoclusters over which the stellar IMF does not globally apply. Therefore, we may expect that the $\geq 40 M_{\odot}$ dense cores of Cygnus $\mathrm{X}$ have a high probability of forming $10-20 M_{\odot}$ of stars, including at least one high-mass star. The detection of maser emission toward a few moderate-mass $\left(\sim 20 M_{\odot}\right)$ dense cores that remain weak at mid-infrared wavelength (cf. Wyrowski et al. in prep.) suggests that the $\geq 40 M_{\odot}$ limit for a Cygnus $\mathrm{X}$ dense core to host a high-mass protostar is reasonable.

\subsection{The high potential of Cygnus $X$ dense cores to form massive stars}

The Cygnus $\mathrm{X}$ molecular complex has already formed generations of high-mass stars, since it contains several OB associations (Uyaniker et al. 2001) and numerous H II regions (Wendker et al. 1991). We here discuss its ability to form highmass stars in the near future by making a census of embedded H II regions (Sect. 5.2.1), high-luminosity protostellar cores (Sect. 5.2.2), and massive infrared-quiet cores which are bright in $\mathrm{SiO}$ (Sect. 5.2.3). We summarize in Table 5 the number of objects found at various stages of the high-mass star formation process. 
Table 5. Massive young stellar objects found in Cygnus $X$ at various stages of the high-mass star formation process.

\begin{tabular}{|c|c|c|c|c|c|c|}
\hline & $\begin{array}{l}\text { Starless } \\
\text { clumps }\end{array}$ & $\begin{array}{l}\text { Pre-stellar } \\
\text { cores }\end{array}$ & $\begin{array}{c}\text { Massive IR-quiet } \\
\text { protostars }\end{array}$ & $\begin{array}{l}\text { High-luminosity } \\
\text { IR protostars }\end{array}$ & $\begin{array}{l}\mathrm{H} \text { II } \\
\text { regions }\end{array}$ & OB stars \\
\hline Number in Cygnus $\mathrm{X}$ & $\frac{\mathrm{T}}{10}$ & $\leq 1$ & 17 & 25 & $\sim 800$ & $2600 \pm 1000$ \\
\hline References & this paper & this paper & this paper & this paper & Wendker et al. (1991) & Knödlseder (2000) \\
\hline Statistical lifetime ${ }^{a}$ & $\sim 7 \times 10^{3} \mathrm{yr}$ & $\leq 8 \times 10^{2} \mathrm{yr}$ & $\sim 1.3 \times 10^{4} \mathrm{yr}$ & $\sim 1.9 \times 10^{4} \mathrm{yr}$ & $\sim 6 \times 10^{5} \mathrm{yr}$ & $(2 \pm 1) \times 10^{6} \mathrm{yr}$ \\
\hline$\left\langle n_{\mathrm{H}_{2}}\right\rangle$ & $6.7 \times 10^{3} \mathrm{~cm}^{-3}$ & $\sim 1.5 \times 10^{5} \mathrm{~cm}^{-3}$ & \multicolumn{2}{|c|}{$1.7 \times 10^{5} \mathrm{~cm}^{-3}$} & & \\
\hline Free-fall time ${ }^{b}$ & $4 \times 10^{5} \mathrm{yr}$ & $\sim 9 \times 10^{4} \mathrm{yr}$ & \multicolumn{2}{|c|}{$8 \times 10^{4} \mathrm{yr}$} & & \\
\hline Number in Orion & ? & $\frac{7}{?}$ & $\sim 1$ & $\sim 1$ & $\sim 6$ & $\sim 81$ \\
\hline
\end{tabular}

${ }^{a}$ Lifetime of massive starless clumps, pre-stellar cores, infrared-quiet protostars, high-luminosity infrared sources, and $\mathrm{H}$ II regions measured relative to the known age of OB stars (cf. Hanson 2003) using the census given in Line 3.

${ }^{b}$ Free-fall time measured from the mean values of the volume-averaged density given in Line 6: $t_{\text {free-fall }}=\sqrt{\frac{3 \pi}{32 G\langle\rho\rangle}}$.

\subsubsection{Embedded $\mathrm{H}$ II regions}

Within our sample of high-density cores, we identify 15 embedded $\mathrm{H}$ II regions from their bright infrared and centimeter continuum emissions (see Cols. 8, 9 of Table 1). Most of them are already recognized as compact or ultra-compact $\mathrm{H}$ II regions (Downes \& Rinehart 1966; Haschick et al. 1981; Kurtz et al. 1994; Molinari et al. 1998b; Trinidad et al. 2003). Five others (CygX-N47/DR21-D, CygX-N58/IRAS 20375+4109, CygXS18/S106-IR, S20, CygX-S27/MSX 77.9550+0.0058) coincide with radio centimeter sources from the $1.4 \mathrm{GHz}$ Galactic Plane Survey (Zoonematkermani et al. 1990) or old centimeter maps (Harris 1973; Pipher et al. 1976). As expected, observations of high-density tracers such as $\mathrm{CS}, \mathrm{NH}_{3}$ line and $350 \mu \mathrm{m}$ continuum made toward these $1.2 \mathrm{~mm}$ sources suggest some free-free contamination (e.g. Motte et al. in prep.).

Despite harboring an $\mathrm{H}$ II region, most of these massive young stellar objects are embedded and some of them (e.g. CygX-N46/DR21, CygX-N30/W75(N), CygX-S/AFGL 2591) even show high levels of ongoing star formation activity, such as maser emission and/or powerful outflow shocks (see Tables 1,3 and Figs. 5, 8). In agreement with these $\mathrm{H}$ II regions being compact, ultra-compact, or even hyper-compact, our $1.2 \mathrm{~mm}$ study is sensitive to young and thus "embedded H II regions".

\subsection{2. "High-luminosity infrared sources"}

Dense cores which are luminous at infrared wavelengths are usually considered to be the best candidates to be (or to host) highmass protostars (e.g. Wood \& Churchwell 1989). Following a similar philosophy, we qualify as "high-luminosity infrared sources" those Cygnus X dense cores with bolometric luminosity larger than $10^{3} L_{\odot}$, which corresponds to that of a B3 star on the main sequence. This luminosity converts into a MSX $21 \mu \mathrm{m}$ flux of $\sim 10 \mathrm{Jy}$, assuming that the luminosity of high-mass protostars is dominated by their mid- to far-infrared luminosity and their average colors are defined by Wood \& Churchwell (1989, see their Table 1). In practice, we estimate the total flux in the IRAS bands using the simplified formulae ${ }^{6}$ of Casoli et al. (1986) and the Wood \& Churchwell (1989) colors to express this total flux as a function of the single IRAS $25 \mu \mathrm{m}$ flux. We finally convert the $25 \mu \mathrm{m}$ flux into an expected $M S X$ flux at $21 \mu \mathrm{m}$ by an interpolation between 25 and $12 \mu \mathrm{m}$ using the Wood \& Churchwell color. For $d=1.7 \mathrm{kpc}$, we finally estimate a typical $M S X$ flux at $21 \mu \mathrm{m}$ of $\sim 10 \mathrm{Jy} \times\left(L_{\mathrm{IRAS}} / 10^{3} L_{\odot}\right)$ for a B3 protostar. Figure 7 displays the $21 \mu \mathrm{m}$ flux detected toward Cygnus $\mathrm{X}$

${ }^{6} F_{\text {IRAS }}=1.75 \times 10^{-13} \mathrm{~W} \mathrm{~m}^{-2} \times\left(S_{12} / 0.79+S_{25} / 2+S_{60} / 3.9+S_{100} / 9.9\right)$ with $S_{12}, S_{25}, S_{60}, S_{100}$ expressed in Jy.

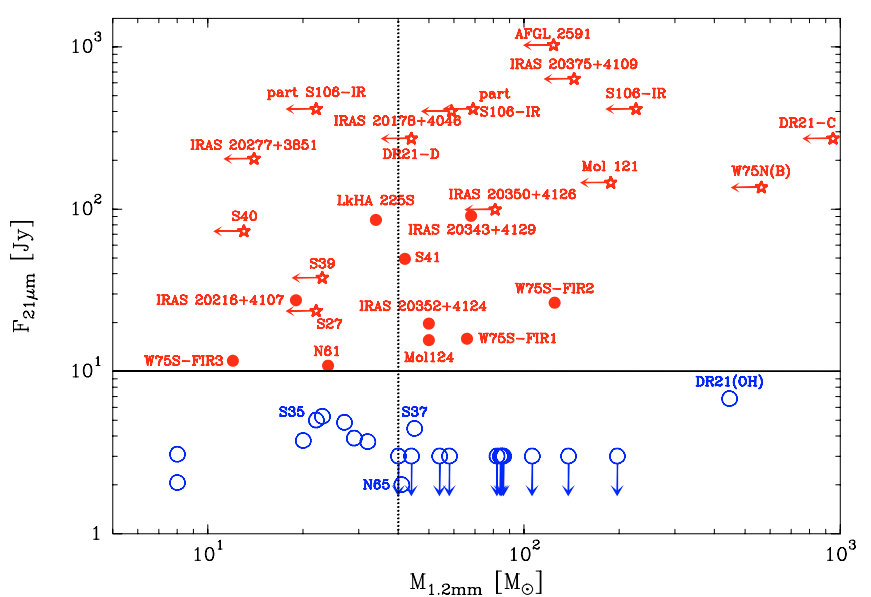

Fig. 7. Separating the high-luminosity $\left(>10^{3} L_{\odot}\right)$ sources from infraredquiet cores using their $21 \mu \mathrm{m}$ flux (limit set to $10 \mathrm{Jy}$ ). In Cygnus X, $15 \mathrm{UCH}$ II regions (red star-like markers) and 10 infrared protostars (red filled circles) have high luminosity. A few infrared-quiet cores (blue open circles) are weak $M S X$ sources (like DR21(OH)) but most of them remain undetected (those more massive than $40 M_{\odot}$ are plotted here).

dense cores as a function of their mass. With a $10 \mathrm{Jy}$ limit in Fig. 7, we detect all the embedded $\mathrm{H}$ II regions plus 10 sources which are "high-luminosity infrared protostellar cores". Three of the latter are associated with $\mathrm{OH}, \mathrm{H}_{2} \mathrm{O}$, and/or $\mathrm{CH}_{3} \mathrm{OH}$ maser emission: CygX-N43/W75S-FIR1, CygX-N44/DR21(OH), and CygX-NW5/LkA 225S.

The high-luminosity infrared sources of the present Cygnus X survey are mostly IRAS sources fulfilling the Wood \& Churchwell (1989) criteria for UCH II regions or objects in an earlier stage of high-mass star formation. Many of them were already identified as high-mass protostellar sources: e.g. W75S-FIR1 and -FIR2 (see Chandler et al. 1993a) and IRAS 20343+4129 and IRAS 20216+4107 (Beuther et al. 2002).

\subsubsection{Strong $\mathrm{SiO}$ emission as a probe for high-mass protostars}

In contrast to high-luminosity infrared sources, massive infrared-quiet cores are not all expected to host high-mass protostars. It is therefore interesting and somewhat surprising to find that all of them are bright $\mathrm{SiO}$ emitters (cf. Table 3 and Fig. 5). As shown below, their $\mathrm{SiO}(2-1)$ lines are well interpreted as due to a powerful outflow that is probably driven by one high-mass protostar.

As shown in Fig. 8, which presents the $\mathrm{SiO}$ intensity of the Cygnus $\mathrm{X}$ dense cores versus their mass, the $\mathrm{SiO}$ lines detected for infrared-quiet cores are several times brighter than 


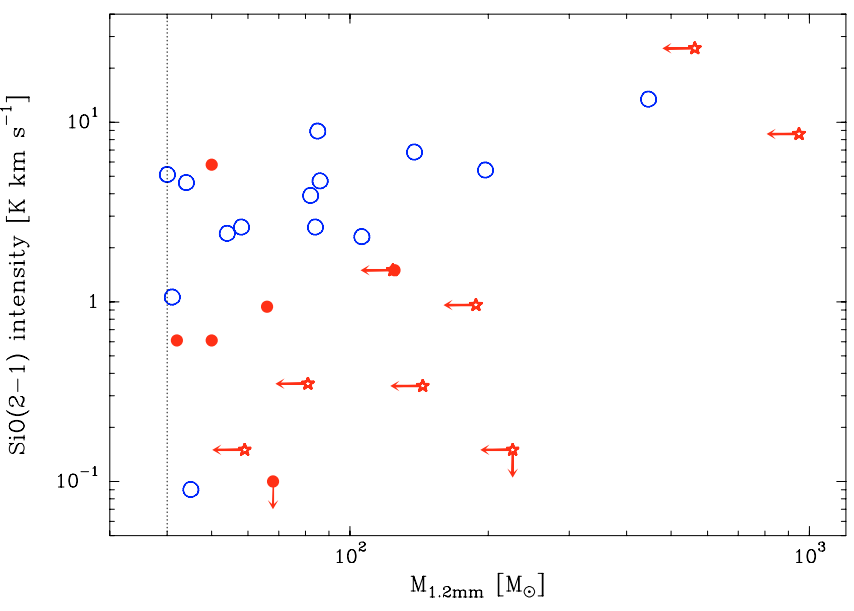

Fig. 8. Integrated intensity of $\mathrm{SiO}(2-1)$ detected toward the Cygnus $X$ dense cores more massive than $40 M_{\odot}$, as a function of their mass. Infrared-quiet cores (blue open circles) are definitely stronger in $\mathrm{SiO}$ than the high-luminosity protostellar cores (red filled circles) and UCH II regions (red star-like markers).

those observed for the high-luminosity sources. As an example, the well-recognized UCH II region CygX-S26/AFGL 2591 is weaker (in $\mathrm{SiO}$ ) than a completely unknown source such as CygX-N53. Since (SiO) outflows are tracing the accretion process (see Bontemps et al. 1996), this result suggests that massive infrared-quiet cores are in a more active accretion phase than high-luminosity infrared sources. Furthermore, the SiO emission of massive infrared-quiet cores is several times stronger than the most extreme $\mathrm{SiO}$ shocks associated with nearby lowmass class 0 protostars (see e.g. Table 3 ). This suggests that the infrared-quiet cores host either a clear single $\mathrm{SiO}$ outflow driven by a high-mass protostar, or a group of $\mathrm{SiO}$ outflows as extreme as those of L1448-MM or L1157-MM. Quantitatively the mean $\mathrm{SiO}$ brightness of the massive infrared-quiet cores in Cygnus $\mathrm{X}$ would require the summation of two low-mass class 0s driving extreme $\mathrm{SiO}$ outflows (and up to 5 in the case of CygX-N53). This is rather improbable, since we evaluate the occurence of such $\mathrm{SiO}$ outflows to be of the order of $6 \%$ ( 2 out of 35 class $0 \mathrm{~s}$ known at $d<500$ pc; e.g. André et al. 2000). Statistically, the summation of several tens of low-mass class 0s would be required to explain the bright $\mathrm{SiO}$ emissions observed here. The $\mathrm{SiO}$ outflows of these infrared-quiet cores better resemble that of the high-mass protostar Orion-IRc2 (see Ziurys \& Friberg 1987, and Table 3). These intensity comparisons suggest that the infrared-quiet protostellar cores of Cygnus $\mathrm{X}$ are much more active (in star formation) than nearby low-mass protostars, and that a large fraction of them should host high-mass analogs of class $0 \mathrm{~s}$.

The profile of the $\mathrm{SiO}$ lines provides us with useful additional constraints. Half of the infrared-quiet protostellar cores in Table 3 (CygX-N53, N63, N68, S30, S32 and possibly $\mathrm{N} 3, \mathrm{~N} 65, \mathrm{~S} 7$ ) show clear and smooth outflow wings in their $\mathrm{SiO}$ line profiles. These profiles are so clean that it can be taken as a good indication that the $\mathrm{SiO}$ emission is dominated by a single $\mathrm{SiO}$ outflow which, in turn, has to be powered by a high-mass protostar. Four infrared-quiet protostellar cores (CygX-N44/DR21(OH), CygX-N48/DR21(OH)-S, CygX$\mathrm{N} 38 / \mathrm{DR} 21(\mathrm{OH})-\mathrm{W}$, and CygX-N12) have a profile which is more indicative of two emission components: a strong narrow line plus a line with large wings (see Sect. 4.2). This can arise from two different $\mathrm{SiO}$ outflows which would both still be powerful, or from a single outflow composed of two main velocity patterns in the $\mathrm{SiO}$ shocks. An alternative interpretation is that, besides the outflow, some shocks associated with a hot core would be detected as a narrow $\mathrm{SiO}$ line component.

Therefore, the $\geq 40 M_{\odot}$ dense cores of Cygnus $\mathrm{X}$ all have a high probability of containing (at least) one high-mass protostar, whether or not they are bright infrared sources. Our selection of extreme-density cores has thus proven to be very efficient in locating sites of future formation of high-mass stars.

\section{3. "Massive infrared-quiet protostars": definition and lifetime}

\subsubsection{Definition}

We define as "massive infrared-quiet protostellar cores" the Cygnus X dense cores that are more massive than $40 M_{\odot}$, weak at infrared wavelengths (i.e. $<10 \mathrm{Jy}$ at $21 \mu \mathrm{m}$ ), but present some signposts of stellar activity. Table 1 provides a list of 33 dense cores more massive than $40 M_{\odot}$ (see also Table 4) and Fig. 7 allows the identification of "high-luminosity infrared sources" (see also Sect. 5.2.2). The 17 remaining dense cores should either be infrared-quiet protostellar cores or starless cores. Among them, four are already identified as protostellar cores by their weak $M S X$ detection and/or their association with masers: CygX-N44/DR21(OH), CygX-N65, CygXS37/IRAS 20305+4010, and CygX-N53. The majority of the 17 massive infrared-quiet cores have been surveyed in $\mathrm{SiO}(2-1)$; 14 positions are listed in Table 3 and CygX-N32 is included in the beam targeting CygX-N30. The brightness and line shape of their $\mathrm{SiO}$ emission prove that the infrared-quiet cores are all protostellar in nature and that they probably host a high-mass protostar (see Sect. 5.2.3).

\subsubsection{Lifetime of high-mass protostars}

Our study supports the existence of massive infrared-quiet protostars, overlooked by infrared-based surveys (see Figs. 7, 8). It provides a sample of (mostly) new massive infrared-quiet protostellar cores which contain high-mass protostars. We still lack the necessary angular resolution to identify individual high-mass protostars, but the characteristics of each Cygnus X dense core more massive than $40 M_{\odot}$ are believed to be shaped by those of a single embedded high-mass protostar (cf. Sects. 5.1 and 5.2.3). With this assumption, we give the first lifetime estimates for this new population of high-mass protostars, assuming a constant star formation rate in Cygnus X over the past 1-2 Myr.

Since our sample of Cygnus $\mathrm{X}$ dense cores is complete for embedded, massive, young stellar objects, we can statistically estimate the relative lifetimes of massive infrared-quiet protostars and high-luminosity infrared sources (including infrared protostars and embedded H II regions). The separation of infrared sources between embedded $\mathrm{H}$ II regions and highluminosity infrared cores is not final since centimeter continuum follow-up observations are necessary, and a low-level of centimeter free-free detection might be indicative of outflow shocks rather than $\mathrm{H}$ II regions. In the following discussion, we therefore treat cores hosting high-luminosity infrared sources and possibly protostars as a single population. According to the numbers of high-luminosity infrared sources and massive infrared-quiet protostars given in Table 5 (and taken from Sects. 5.2.1-5.2.2 and Sect. 5.3.1), the infrared-quiet protostellar phase of high-mass stars might last as long as their betterknown infrared-bright protostellar (or embedded H II) phase. This result is in disagreement with the idea that high-mass 


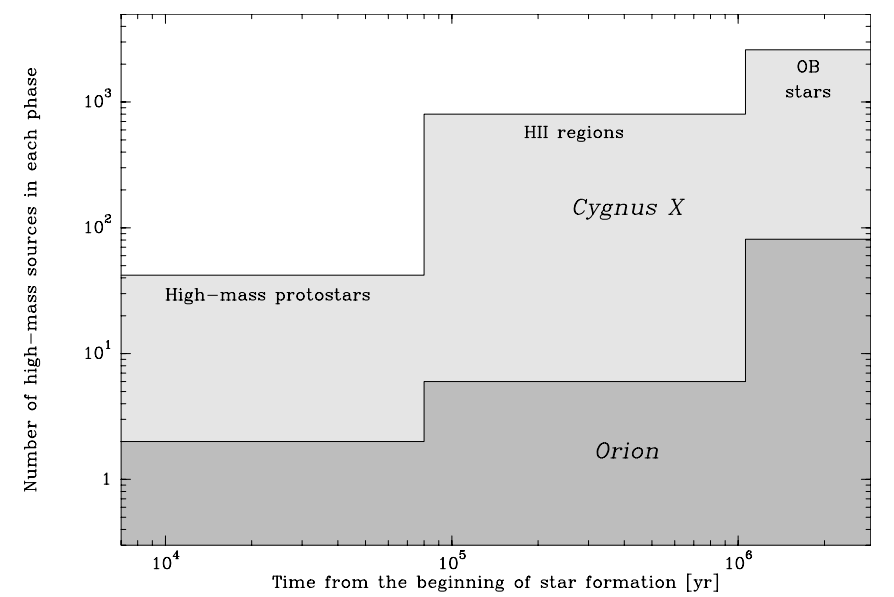

Fig. 9. Number of massive young stellar objects as a function of the time elapsed since the beginning of the protostellar collapse in Cygnus $\mathrm{X}$ and Orion. The age estimates (also given in Sect. 5.3.2) are not statistical, in order to have the $X$-axis and $Y$-axis independent.

protostars are very luminous from the very first stage of their evolution and thus that their infrared-quiet phase is very shortlived.

Table 5 gives our current estimates of the number of massive young stellar objects forming and stars already formed in Cygnus X. The number and age of OB stars in Cygnus X are taken to be those of the Cyg OB2 association (Knödlseder 2000; Hanson 2003), increasing uncertainties to take into account the other (smaller) OB associations which have different ages (Uyaniker et al. 2001). The number of $\mathrm{H}$ II regions is crudely given by the numbers of centimeter free-free sources observed in Cygnus X (Wendker et al. 1991). The statistical lifetimes of high-mass protostars derived relative to the number of OB stars give a time range of $(0.5-3.1) \times 10^{4} \mathrm{yr}$ for the infraredquiet protostellar stage and $(1.2-7.9) \times 10^{4} \mathrm{yr}$ for the complete protostellar stage (see also Table 5). For the sake of comparison, the statistical lifetime measured for the high-mass protostellar phase in Cygnus X $\left(\sim 3.2 \times 10^{4} \mathrm{yr}\right)$ is one order of magnitude smaller than the typical age of nearby, low-mass class I protostars (e.g. Kenyon \& Hartmann 1995) and more closely resembles that of low-mass class 0s (cf. André et al. 2000).

Figure 9 displays the number of high-mass protostars, $\mathrm{H}$ II regions and $\mathrm{OB}$ stars (i.e. objects in the three main evolutionary phases) as a function of the time elapsed since the beginning of the protostellar collapse (i.e. since the formation of a hydrostatic stellar embryo). Absolute lifetimes of the highmass protostellar phase (encompassing both the infrared-quiet and the high-luminosity infrared protostellar phases) as well as those of the high-mass starless phases can be roughly estimated from their free-fall dynamical timescales. Table 5 gives the estimates we made using the mean values of the volume-averaged density of massive dense cores observed in Cygnus $\mathrm{X}$. The most massive dense cores of Cygnus $X$ should thus collapse in a freefall time of $\sim 8 \times 10^{4} \mathrm{yr}$, which is taken to be the end of the high-mass protostellar phase in Fig. 9. The $\mathrm{H}$ II regions phase should last for several times the typical lifetime of UCH II regions $\left(\sim 10^{5} \mathrm{yr}\right.$, cf. Wood \& Churchwell 1989), starting in Fig. 9 at the end of the protostellar phase. The free-fall time of highmass protostars in Cygnus $\mathrm{X}$ agrees, within a factor of 3 , with the statistical lifetimes of the complete population of high-mass protostars (including both infrared-quiet and high-luminosity infrared protostars) derived relative to the number of OB stars (see Table 5).
To compare Cygnus $X$ to Orion, Table 5 makes the census of all massive young stellar objects known in Orion, and Fig. 9 shows their distribution as a function of time. The number of high-mass stars (down to B3 spectral type) within all the Orion OB associations is measured by Brown et al. (1994), while the $\mathrm{H}$ II regions are associated with the well-known reflection nebulae M42, NGC 2024, NGC 1977, M43, NGC 2068, and NGC 2071. One source could qualify as a high-luminosity infrared protostar (Orion-IRC2) and another as a massive infraredquiet protostar (OMC1-S). Interestingly, the massive young stellar objects forming, and stars formed, in Cygnus $\mathrm{X}$ are 20 times more numerous than in Orion (see Table 5), in good agreement with the mass ratio (13) of the Cygnus $\mathrm{X}$ and Orion cloud complexes.

\subsection{Where is the pre-stellar phase of massive star formation?}

Among the 33 most massive $\left(\geq 40 M_{\odot}\right)$ dense cores of Cygnus X, no starless cores are found, with the possible exception of CygXN69 which was not selected for $\mathrm{SiO}$ observations because of its relatively large size and corresponding low-density $\left(\sim 10^{4} \mathrm{~cm}^{-3}\right)$. This non-detection of starless cores means that high-mass prestellar cores, if they exist, are short-lived $\left(<10^{3} \mathrm{yr}\right.$, see Table 5). This is particularly surprising since the lifetime of pre-stellar cores and condensations in nearby, low-mass star-forming regions is more than two orders of magnitude longer $\left(1-4 \times 10^{5} \mathrm{yr}\right.$, e.g. Onishi et al. 2002; Kirk et al. 2005; André et al. 2007). Below, we consider two interpretations: either the star formation activity of Cygnus $X$ is not continuous (see Sect. 5.4.1) or massive pre-stellar clumps are dynamically evolving into protostars (see Sect. 5.4.2).

\subsubsection{Star formation in Cygnus $X$ cannot be caused by a burst}

If the Cygnus $\mathrm{X}$ molecular complex were experiencing a sudden burst of star formation, it might be due to the interaction with the $\mathrm{H}$ II regions and/or OB associations, including the Cyg OB2 cluster. In this case, we expect differences in the concentration of gas and in the population of young stellar objects in the CygXNorth and CygX-South regions.

Evidence shows that the concentration of gas is different in CygX-North and CygX-South. The CO survey of Schneider et al. (2006), the present MAMBO-2 maps, and our small-scale analysis trace various cloud structures (see Sects. 3.1-3.2). We can thus measure the gradual concentration of molecular clouds (0.5-50 pc scales), first into $0.1-5 \mathrm{pc}$ scales, and then into $0.1 \mathrm{pc}$ dense cores. In CygX-North $\left(280 \times 10^{3} M_{\odot}\right.$, cf. Schneider et al. 2006), the concentration factor is 8 for each step while in CygXSouth $\left(480 \times 10^{3} M_{\odot}\right)$ it is 18 for each step. Therefore, when considering the mass involved in dense cores, the CygX-North region is 5 times more concentrated ( $\mathrm{CO}$ clouds concentrate into $0.1 \mathrm{pc}$ cores with an efficiency of $\sim 1.5 \%$ ) than CygX-South (concentration efficiency of $\sim 0.3 \%$ ). This suggests that CygX-North could be 5 times more efficient in forming dense cores, and possibly forming stars, than CygX-South.

In contrast, the relative numbers of "high-luminosity infrared sources" versus "massive infrared-quiet protostellar cores" are identical (within our large error bars) in both regions. Moreover, other generations of (massive) young stars have already formed nearby (see e.g. the $M S X$ sources at $\sim 0.2$ pc from the dense cores). The agreement between the free-fall lifetime and the statistical lifetime of high-mass protostars relative to OB stars also suggests that star formation has been continuous, at least over 
the past $\sim 10^{6} \mathrm{yr}$ (see also Fig. 9). Therefore, it is highly improbable that Cygnus $\mathrm{X}$ is currently experiencing a burst of star formation.

\subsubsection{Is the star formation in Cygnus $X$ dynamic?}

The present study fails to find a single good candidate for being a pre-stellar dense core, i.e. a starless cloud structure with a $\sim 0.1 \mathrm{pc}$ size and a $\sim 10^{5} \mathrm{~cm}^{-3}$ volume-averaged density, which is gravitationally bound. This does not preclude, however, the existence of smaller and denser pre-stellar condensations $(\sim 0.01 \mathrm{pc}$ size and $\sim 10^{6} \mathrm{~cm}^{-3}$ density according to Motte et al. 1998, see Table 4) within the Cygnus $\mathrm{X}$ protostellar cores. On the other hand, we have identified 10 clumps which may be starless ( 0.8 pc size and $7 \times 10^{3} \mathrm{~cm}^{-3}$ density, see Tables 2 and 4$)$. The Virial mass of these clumps $\left(M_{\mathrm{vir}}=3 R \sigma^{2} / G\right.$, Bertoldi \& McKee 1992) are estimated from the $\mathrm{N}_{2} \mathrm{H}^{+}$linewidth measured at their peak location (Schneider et al. in prep.) and the FWHM size given in Table 2; they suggest that most of these clumps are close to being gravitationally bound.

From the numbers of starless clumps and pre-stellar cores detected in Cygnus $\mathrm{X}$ and compared to the numbers of OB stars, we statistically estimate their lifetimes to be $\sim 10^{4} \mathrm{yr}$ and $<10^{3} \mathrm{yr}$, respectively (see Table 5 ). These lifetime values are considerably smaller (by factors of 60-110) than the freefall times we estimated. They are also shorter than the lifetimes observed in nearby regions that form mostly low-mass stars, since starless cloud structures with volume-averaged densities of $\sim 10^{3} \mathrm{~cm}^{-3}$ and $\sim 10^{4} \mathrm{~cm}^{-3}$ have pre-stellar lifetimes of $\sim 10^{6}$ yr and $\sim 10^{5} \mathrm{yr}$ (see Fig. 11 of Kirk et al. 2005, and references therein). Therefore, massive pre-stellar cores seem to be short-lived or even transient cloud structures compared to nearby low-mass pre-stellar cores, to high-mass protostars, and to massive starless clumps.

A dynamical process should thus be acting to create prestellar condensations from starless clumps. It should be supersonic to concentrate mass from densities of $7 \times 10^{3} \mathrm{~cm}^{-3}$ to $2 \times 10^{5} \mathrm{~cm}^{-3}$ in a time as short as, or even shorter than, the free-fall timescale. Similarly short lifetimes are theoretically expected in molecular clouds where highly turbulent processes dominate and pre-stellar cores are magnetically supercritical (e.g. Vázquez-Semadeni et al. 2005). Such dynamical processes are also necessary for the protostellar lifetime to last for only one free-fall time (see Table 5) and, consequently, for the accretion to be strong enough $\left(\sim 10^{-3} M_{\odot} \mathrm{yr}^{-1}\right)$ to overcome the radiation pressure and form a high-mass star. In fact, the DR21 filament is observed to undergo a global supersonic collapse, possibly due to a strong external compression (Chandler et al. 1993b; Motte et al. 2005). This is encouraging but it remains to be verified whether such a dynamical process acts throughout the complex.

Taken together, the lifetimes measured here suggest a faster evolution (by a factor 10 at least) of the pre-stellar and protostellar phases of high-mass stars compared to those typically found for nearby, low-mass stars. Molecular clouds harboring high-mass star formations should thus be quantitatively different in terms of e.g. density and kinematics than those of our close neighborhood.

\section{Conclusions}

In order to improve our knowledge of the high-mass star formation process, we started an unbiased search for its earliest phases, i.e. the high-mass analogs of low-mass pre-stellar cores and class 0 protostars. One of the best targets for such a study is Cygnus X, which we recently recognized as the richest molecular and $\mathrm{H}$ II complex at less than $3 \mathrm{kpc}$ (it is located at $1.7 \mathrm{kpc}$ from the Sun). We have therefore made a complete $1.2 \mathrm{~mm}$ continnum survey of the Cygnus $\mathrm{X}$ cloud complex and have performed $\mathrm{SiO}(2-1)$ follow-up observations of the best candidate progenitors of high-mass stars. Our main findings can be summarized as follows:

1. Our MAMBO-2 (1.2 $\mathrm{mm}, 11^{\prime \prime}$ resolution) imaging of the Cygnus X molecular cloud complex gives a complete view of the cloud structures ranging from 0.03 to $5 \mathrm{pc}$, i.e. from dense cores to clumps (cf. the terminology used for nearby molecular clouds). We performed a multi-resolution analysis to identify a few tens of massive large-scale $(\sim 0.7 \mathrm{pc})$ clumps and extract 129 compact $(\sim 0.1 \mathrm{pc})$ dense cores.

2. The Cygnus X dense cores are the high-density parts of massive clumps which resemble HMPOs or IRDCs. The dense cores have similar sizes $(\sim 0.1 \mathrm{pc})$ but mass and volumeaveraged densities $\left(4-950 M_{\odot}, \sim 1.1 \times 10^{5} \mathrm{~cm}^{-3}\right)$ larger than those of nearby, pre-stellar dense cores. The most massive $\left(\geq 40 M_{\odot}\right)$ and thus "extreme-density" cores of this large and unbiased sample are probable precursors of high-mass stars spanning, for the first time, the whole evolution from prestellar cores to "embedded H II regions".

3. We have used the MSX $21 \mu \mathrm{m}$ flux arising from our MAMBO-2 dense cores to identify the high-luminosity $\left(>10^{3} L_{\odot}\right)$ massive young stellar objects of Cygnus $\mathrm{X}$ : $15 \mathrm{UCH}$ II regions and 10 "high-luminosity infrared protostars". Strikingly, half of the dense cores considered as the best candidate precursors of high-mass stars are found to be infrared-quiet (i.e. 17 Cygnus $\mathrm{X}$ dense cores more massive than $40 M_{\odot}$ have $F_{21 \mu \mathrm{m}}<10 \mathrm{Jy}$ ).

4. We have surveyed the most massive $\left(\geq 40 M_{\odot}\right)$ dense cores of Cygnus $\mathrm{X}$ in $\mathrm{SiO}(2-1)$ to search for shocked gas in molecular outflows and/or hot cores. The association of highvelocity $\mathrm{SiO}$ emission with all massive infrared-quiet cores provides persuasive evidence that stars are already forming in these cores as outflows are tracing the accretion process. The brightness and shape of the $\mathrm{SiO}$ line compared to that of nearby, low-mass protostars suggest that these extremedensity cores may host "massive infrared-quiet protostars".

5. Our unbiased survey of the massive young stellar objects in Cygnus $\mathrm{X}$ shows that massive infrared-quiet protostars do exist, and that their lifetime should be comparable to that of more evolved high-luminosity infrared protostars. By comparing the number of high-mass protostars and OB stars in the whole of Cygnus X, we estimate a statistical lifetime of $3 \times 10^{4} \mathrm{yr}$ for high-mass protostars. One order of magnitude smaller than the lifetime of nearby, low-mass protostars, such a value agrees, within a factor of 3 , with the free-fall time of Cygnus $\mathrm{X}$ dense cores.

6. Our complete census of massive young stellar objects in Cygnus $\mathrm{X}$ fails to discover the high-mass analogs of prestellar dense cores $\left(\sim 0.1 \mathrm{pc},>10^{4} \mathrm{~cm}^{-3}\right)$. Their corresponding lifetime $\left(<10^{3} \mathrm{yr}\right)$ is smaller than one free-fall time, in marked contrast with nearby, pre-stellar cores that typically live for 2-5 free-fall times. We propose that the starless, massive but lower-density clumps $\left(800 M_{\odot}, \sim 0.8 \mathrm{pc}\right.$, $\sim 7 \times 10^{3} \mathrm{~cm}^{-3}$ ) that we observe rapidly concentrate and collapse to form high-mass protostars.

7. Lifetime measurements of the pre-stellar and protostellar phases of high-mass stars in Cygnus $\mathrm{X}$ suggest a dynamical contraction for pre-stellar cores and a supersonic evolution for protostars. Highly turbulent processes throughout the Cygnus X molecular cloud complex would be necessary 
in such a dynamical picture of the high-mass star formation process.

8. Our Cygnus $\mathrm{X}$ study suggests that far-infrared to submillimeter continuum imaging of entire star-forming complexes (such as those proposed with Herschel by Motte, Zavagno, Bontemps et al.: the $\mathrm{HOBYS}^{7}$ Key Programme) will definitely contribute to a better knowledge of the highmass star formation process during its earliest phases.

Acknowledgements. We are grateful to Axel Weiss, Roberto Neri and Clemens Thum for their help in taking and reducing the MAMBO data. We thank Henrik Beuther and Jill Rathborne for providing electronic versions of their tables, as well as Friedrich Wyrowski for unveiling his Effelsberg results before publication. We are grateful to Tom Megeath for useful discussions on Orion and Arnaud Belloche for the suggestion of using volume-averaged densities.

\section{References}

Abergel, A., Bernard, J. P., Boulanger, F., et al. 2002, A\&A, 389, 239 André, P., Ward-Thompson, D., \& Barsony, M. 2000, in Protostars \& Planets IV, ed. V. Mannings, A. Boss, \& S. Russell (Tucson: Univ. Arizona Press), 59 André, P., Belloche, A., Motte, F., \& Peretto, N. 2007, A\&A, 472, 519 Argon, A. L., Reid, M. J., \& Menten, K. M. 2000, ApJS, 129, 159 Bertoldi, F., \& McKee, C. F. 1992, ApJ, 395, 140

Beuther, H., Schilke, P., Menten, K. M., et al. 2002, ApJ, 566, 945 Bonnell, I. A., Bate, M. R., Clarke, C. J., \& Pringle, J. E. 2001, MNRAS, 323, 794

Bontemps, S., Motte, F., Schneider, N., in prep.

Bontemps, S., André, P., Kaas, A. A., et al. 2001, A\&A, 372, 173

Bontemps, S., André, P., Terebey, S., \& Cabrit, S. 1996, A\&A, 311, 858

Braz, M. A., \& Epchtein, N. 1983, A\&AS, 54, 167

Broguière, D., Neri, R., \& Sievers, A. 1995, NIC bolometer users guide, IRAM internal report

Bronfman, L., Nyman, L.-A., \& May, J. 1996, A\&AS, 115, 81

Brown, A. G. A., de Geuss, E. J., \& de Zeeuw, P. T. 1994, A\&A, 289, 101

Carey, S. J., Feldman, P. A., Redman, R. O., et al. 2000, ApJ, 543, L157

Casoli, F., Combes, F., Dupraz, C., Gerin, M., \& Boulanger, F. 1986, A\&A, 169, 281

Chandler, C. J., Gear, W. K., \& Chini, R. 1993, MNRAS, 260, 337

Chandler, C. J., Moore, T. J. T., Mountain, C. M., \& Yamashita, T. 1993, MNRAS, 261, 694

Churchwell, E. 1999, in The Origin of Stars and Planetary Systems, ed. C. J. Lada, \& N. D. Kylafis (Kluwer Academic Publishers), 515

Davis, C. J., Kumar, M. S. N., Sandell, G., et al. 2007, MNRAS, 374, 29

Downes, D., \& Rinehart, R. 1966, ApJ, 144, 937

Egan, M. P., Price, S. D., Kraemer, K. E., et al. 2003, VizieR On-line Data Catalog, originally published in: Air Force Research Lab. Technical Rep.

Emerson, D. T., Klein, U., \& Haslam, C. G. T. 1979, A\&A, 76, 92

Faúndez, S., Bronfman, L., Garay, G., et al. 2004, A\&A, 426, 97

Hanson, M. 2003, ApJ, 597, 957

Harris, S. 1973, MNRAS, 162, Short Communication

Haschick, A. D., Reid, M. J., Burke, B. F., Moran, J. M., \& Miller, G. 1981, ApJ, 244, 76

Hatchell, J., Fuller, G. A., \& Millar, T. J. 2001, A\&A, 372, 281

Helmich, F. P., \& van Dishoek, E. F. 1997, A\&AS, 124, 205

Henning, T., Michel, B., \& Stognienko, R. 1995, Planet. Space Sci., Special issue: Dust, molecules and backgrounds, 43, 1333

Hill, T., Burton, M. G., Minier, V., et al. 2005, MNRAS, 363, 405

Kenyon, S. J., \& Hartmann, L. W. 1995, ApJS, 101, 117

Kirk, J. M., Ward-Thompson, D., \& André, P. 2005, MNRAS, 360, 1506

Knödlseder, J. 2000, A\&A, 360, 539

Kramer, C., Stutzki, J., Rohrig, R., \& Corneliussen, U. 1998, A\&A, 329, 249

Kreysa, E., Gemünd, H. P., Gromke, J., et al. 1998, in Advanced Technology MMW, Radio, and Terahertz Telescopes, ed. T. G. Phillips, SPIE, 3357, 319 Krumholz, M. R., Klein, R. I., \& McKee, C. F. 2007, ApJ, 656, 959

Kurtz, S., Cesaroni, R., Churchwell, E., Hofner, P., \& Walmsley, C. M. 2000, in Protostars \& Planets IV, ed. V. Mannings, A. Boss, \& S. Russell, 299 Kurtz, S., Churchwell, E., \& Wood, D. O. S. 1994, ApJS, 91, 659

Leung, H. O., \& Thaddeus, P. 1992, ApJS, 81, 267
Marston, A. P., Reach, W. T., Noriega-Crespo, A., et al. 2004, ApJS, 154, 333 Martín-Pintado, J., Bachiller, R., Fuente, A. 1992, A\&A, 254, 315

Minier, V., Conway, J. E., \& Booth, R. S. 2001, A\&A, 369, 278

Molinari, S., Testi, L., Brand, J., Cesaroni, R., \& Palla, F. 1998a, ApJ, 505, L39

Molinari, S., Brand, J., Cesaroni, R., Palla, F., \& Palumbo, G. G. C. 1998b, A\&A, 336, 339

Molinari, S., Brand, J., Cesaroni, R., \& Palla, F. 2000, A\&A, 355, 617

Mookerjea, B., Kramer, C., Nielbock, M., \& Nyman, L.-A. 2004, A\&A, 426, 119

Motte, F., \& André, P. 2001, A\&A, 365, 440

Motte, F., André, P., \& Neri, R. 1998, A\&A, 336, 150

Motte, F., Schilke, P., \& Lis, D. C. 2003, ApJ, 582, 277

Motte, F., Bontemps, S., Schilke, P., et al. 2005, in Massive star birth: A crossroads of Astrophysics, ed. Cesaroni, Felli, Churchwell, \& Walmsley, IAU Symp., 227, 151

Mueller, K. E., Shirley, Y. L., Evans, N. J., II, \& Jacobson, H. R. 2002, ApJS, 143, 469

Nisini, B., Codella, C., Giannini, T., et al. 2007, A\&A, 462, 163

Onishi, T., Mizuno, A., Kawamura, A., Tachihara, K., \& Fukui, Y. 2002, ApJ, 575,950

Ossenkopf, V., \& Henning, T. 1994, A\&A, 291, 943

Palla, F., Brand, J., Cesaroni, R., Comoretto, G., \& Felli, M. 1991, A\&AS, 246, 249

Palla, F., Testi, L., Hunter, T. R., et al. 1995, A\&A, 293, 521

Pestalozzi, M. R., Minier, V., \& Booth, R. S. 2005, A\&A, 432, 737

Pipher, J. L., Sharpless, S., Savedoff, M. P., et al. 1976, A\&A, 51, 255

Plume, R., Jaffe, D. T., Evans, N. J., II, Martín-Pintado, J., \& Gómez-González, J. 1997, ApJ, 476, 730

Rathborne, J. M., Jackson, J. M., \& Simon, R. 2006, ApJ, 641, 389

Reid, M. A., \& Wilson, C. D. 2005, ApJ, 625, 891

Richer, J. S., Padman, R., Ward-Thompson, D., Hills, R. E., \& Harris, A. I. 1993, MNRAS, 262, 839

Sandell, G., \& Sievers, A. 2004, ApJ, 600, 269

Schilke, P., Walmsley, C. M., Pineau des Forets, G., \& Flower, D. R. 1997, A\&A, 321,293

Schneider, N., Bontemps, S., Simon, R., et al. 2006, A\&A, 458, 855

Schneider, N., Simon, R., Kramer, C., Stutzki, J., \& Bontemps, S. 2002, A\&A, 384,225

Shepherd, D. S., Kurtz, S. E., \& Testi, L. 2004, ApJ, 601, 952

Smith, M. D., Eisloffel, J., \& Davis, C. J. 1998, MNRAS, 297, 687

Sridharan, T. K., Beuther, H., Schilke, P., Menten, K. M., \& Wyrowski, F. 2002, ApJ, 566, 931

Starck, J.-L., \& Murtagh, F. 2006, Astronomical image and data analysis, Astronomy and astrophysics library (Berlin: Springer)

Stutzki, J., \& Güsten, R. 1990, ApJ, 356, 513

Teyssier, D., \& Sievers, A. 1998, IRAM internal report

Thompson, M. A., Gibb, A. G., Hatchell, J. H., Wyrowski, F., \& Pillai, T. 2005, in The dusty and molecular universe: a prelude to Herschel and ALMA, ed. A. Wilson, ESA SP-577, 425

Trinidad, M. A., Curiel, S., Cantó, J., et al. 2003, ApJ, 589, 386

Uyaniker, B., Fürst, E., Reich, W., Aschenbach, B., \& Wielebinski, R. 2001, A\&A, 371, 675

Valdettaro, R., Palla, F., Brand, J., et al. 2001, A\&A, 368, 845

Vallée, J. P., \& Fiege, J. D. 2006, ApJ, 636, 332

van der Tak, F. F. S. 2002, in Hot Star Workshop III: The Earliest Phases of Massive Star Birth, ed. P. A. Crowther (San Francisco: ASP), ASP Conf. Ser., 267,33

van der Tak, F. F. S., van Dishoeck, E. F., Evans, N. J., II, Bakker, E. J., \& Blake, G. A. 1999, ApJ, 522, 991

Vázquez-Semadeni, E., Kim, J.. Shadmehri, M., \& Ballesteros-Paredes, J. 2005, ApJ, 618, 344

Ward-Thompson, D., Motte, F., \& André, P. 1999, MNRAS, 305, 143

Wendker, H. J., Higgs, L. A., \& Landecker, T. L. 1991, A\&A, 241, 551

Williams, S. J., Blitz, L., \& McKee, C. F. 2000, in Protostars \& Planets IV, ed.

V. Mannings, A. Boss, \& S. Russell (Tucson: Univ. Arizona Press), 97

Wood, D. O. S., \& Churchwell, E. 1989, ApJ, 340, 265

Yorke, H. W., \& Sonnhalter, C. 2002, ApJ, 569, 846

Ziurys, L. M., \& Friberg, P. 1987, ApJ, 314, L49

Zoonematkermani, S., Helfand, D. J., Becker, R. H., White, R. L., \& Perley, R. A. 1990, ApJS, 74, 181

Zylka, R. 1998, Pocket Cookbook for the MOPSI software
7 "HOBYS: the Herschel imaging survey of OB Young Stellar objects" is a Guaranteed Time Key Programme jointly proposed by the SPIRE and PACS consortia, and the Herschel Science Centre. 
F. Motte et al.: High-mass star formation in the Cygnus X complex, Online Material $p 1$

\section{Online Material}



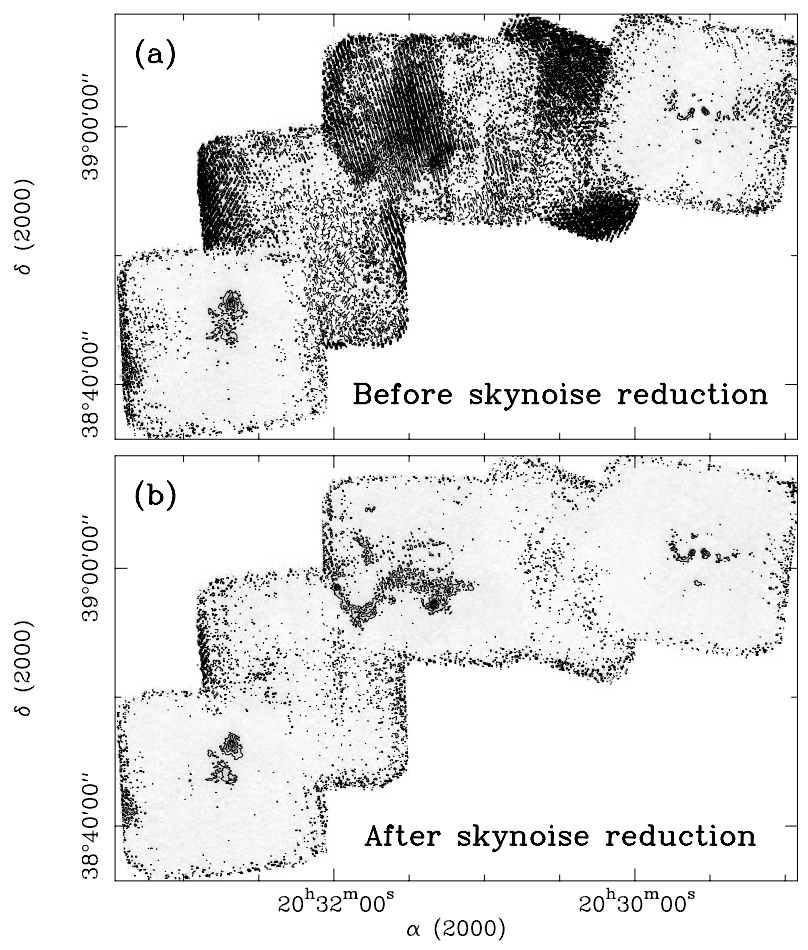

Fig. 10. $1.2 \mathrm{~mm}$ map of the DR13 filament in the Cygnus X molecular cloud complex obtained with MAMBO-2 at the IRAM $30 \mathrm{~m}$ telescope. Contour levels are $-45 \mathrm{mJy}_{\text {beam }}^{-1}$ and then 45 to $315 \mathrm{mJy} \mathrm{beam}^{-1}$ with steps of $45 \mathrm{mJy}^{-1}$ beam ${ }^{-1}$ a) Mosaic built from individual maps taken with low to medium skynoise. As a result, the rms noise level ranges from $15 \mathrm{mJy}$ beam ${ }^{-1}$ to $60 \mathrm{mJy}_{\text {beam }}{ }^{-1}$. b) Mosaic built from individual maps processed by our skynoise reduction program. The resulting noise level is relatively uniform with $\sigma=(14 \pm 2)$ mJy beam $^{-1}$.

\section{Appendix A: A skynoise reduction technique dedicated to extended sources}

Ground-based (sub)millimeter continuum observations of astronomical sources are hampered by the emission of the atmosphere and its fluctuations, called "skynoise". The bulk of the atmospheric emission and its slow variations in time can be removed by the dual-beam scanning mode generally used for bolometer observations (the "EKH method", Emerson et al. 1979). However, the residual atmospheric fluctuations generate an excess noise in the restored maps that can dominate the Gaussian noise (see e.g. Fig. 10a). In the present study, more than half of the on-the-fly maps were observed with a skynoise level qualified as medium or high. In our maps, medium skynoise implies $\sigma=30-60 \mathrm{mJy}$ beam $^{-1}$ and high skynoise $\sigma>60 \mathrm{mJy}$ beam $^{-1}$, while the nominal $\mathrm{rms}$ is $\sigma \sim 15 \mathrm{mJy}^{-1}$ beam $^{-1}$. This excess noise can theoretically be suppressed by using the fact that it is well correlated across the whole array. Several skynoise reduction algorithms have been developed assuming that either the atmospheric emission is equal in all receivers at all times ("mean" or "neighbor" approximation), or that it is correlated on a few seconds of time with correlation coefficients decreasing when the angular separation of receivers increases (see e.g. NIC and MOPSIC IRAM softwares for bolometer-array data, Broguière et al. 1995 and Zylka 1998). While efficient for the detection of pointlike sources, such assumptions can have dramatic consequences on the representation of the extended emission. Indeed, the large-scale structure of a source leads to a correlation of bolometer signals which is non-zero, and caution must be taken not to filter out most of the extended emission.
We have developed a program which uses a source model and the mean approximation to remove the skynoise while preserving, as much as possible, the structure of sources like molecular clouds (compare Figs. 10a, b). We describe below its four steps:

1. The first step consists of creating a good source model. A first-order data processing is done by reducing all individual maps and combining them with weights consistent with their intrinsic quality, i.e. accounting for both Gaussian noise and skynoise levels (see e.g. Fig. 10a). This first-order mosaic is used as a model of the source after cutting its noisier parts (mostly map edges) and removing artefacts such as negative levels stronger than the rms.

2. This source model is then subtracted from all the original scans. To do so, we first simulate the on-the-fly dual-beam signals of each bolometer when the MAMBO-2 camera is mapping the source model with the observational geometry of the real scans (see Motte \& André 2001, and the routine "simulate" in NIC). These simulated source signals are then removed from the original on-the-fly dual-beam signals recorded by each bolometer. At this point, and for each scan, the bolometer data streams should only contain Gaussian noise and skynoise.

3. As a third step, the skynoise emission contained in each processed scan is estimated by using the averaging method. While cruder than any "correlation method" (like that proposed in NIC), it allows the treatment of each observing point (taken every half a second) independently. We often find the averaging method to be more efficient, suggesting that the skynoise power is strong at high frequency.

4. Afterwards, the original scans are reduced for skynoise by subtracting, for each scan, the skynoise data stream estimated above from the on-the-fly dual-beam signals of each bolometer. Finally, the individual scans, now treated for skynoise, are reduced with the default procedure and combined to create a better (second-order processed) mosaic (see e.g. Fig. 10b).

This process can evidently be iterated to improve the map sensitivity but a single iteration already reduces most of the skynoise and probably gives more secure results. The final individual maps have a noise rms of $20 \pm 5 \mathrm{mJy}_{\text {beam }}^{-1}, 15 \mathrm{mJy} \mathrm{beam}^{-1}$ being the nominal rms in the absence of skynoise, and with a zenith atmospheric opacity of $\sim 0.2$. Our skynoise reduction technique has thus improved the map sensitivity by a factor of $\sim 2$ on average, and up to a factor of 6 for the poorer quality original maps.

Our method relies on the quality of the source model and thus mainly on the redundancy of observations taken in every single pixel. The redundancy is currently $\sim 20$ for one single map and $\sim 40$ on average, since most parts were imaged several times. The mosaics shown in Figs. $2 \mathrm{a}-\mathrm{c}$ recover most of the extended emission up to $\sim 10^{\prime}$, which is the azimuthal size of individual maps. In agreement, the Cygnus $\mathrm{X}$ main region has recently been imaged with the SHARC-II camera installed at the CSO telescope (Motte et al. in prep.). Those data generally confirm the cloud structures detected in our MAMBO-2 maps. We acknowledge, however, that the extended emission is attenuated in a couple of sites where data are too noisy and not redundant enough to build a good source model (e.g. south of DR23). 


\section{Appendix B: An extraction technique dedicated to compact sources}

Since stars are generally forming in the densest parts of molecular clouds, we have developed a source extraction technique aiming at identifying and extracting dense compact fragments that should be the best potential sites of star formation.

In spirit, this method is equivalent to an eye search of local density peaks followed by flux measurements with an aperture optimized for each source. We outline below the procedure for this automated method, which was originally developed by Motte et al. (2003):

1. As a first step, one has to determine the cutoff lengthscale at which the sources of interest (here dense cores, cf. Fig. 11b) separate from their surrounding (here clumps and clouds, cf. Fig. 11c). This scale corresponds to twice the mean outer radius of the most compact objects one can distinguish on the MAMBO-2 map or the mean projected distance between them. This scale is typically about 5 to 10 times the $H P B W$.

2. For further analysis, we only keep spatial scales smaller than the cutoff lengthscale determined at step 1 (here $128^{\prime \prime}$ or $1 \mathrm{pc})$. For this purpose, we use a multiresolution program based on wavelet transforms (MRE, cf. Starck \& Murtagh 2006). This makes a series of filtering operations and provides "views" of the image at different spatial scales. We therefore sum $^{8}$ all wavelet planes of the original image up to that scale to create images like that shown in Fig. $11 \mathrm{~b}$.

3. As a third step, we use a version of the Gaussclumps program (Stutzki \& Güsten 1990; Kramer et al. 1998) which has been customized for continuum images (see Motte et al. 2003; and Mookerjea et al. 2004) and optimized for compact sources ${ }^{9}$. Gaussclumps is applied to the filtered image created at step 2. It identifies compact fragments above a $5 \sigma$ level (here $75 \mathrm{mJy}^{-1}$ beam $^{-1}$ ) and gives first-guess parameters of a 2D-Gaussian fit. If the Gaussian shape is not ideally fitting compact fragments, it gives appropriate estimates of their size and mass even when their environment is not perfectly filtered out. Gaussclumps is known to be efficient at deblending compact sources. Noisy edges that usually pose problems (see e.g. Kramer et al. 1998) have been removed.

4. Finally, the most critical and time consuming step is to improve the extraction of individual fragments from their surrounding background. The main goal is to avoid Gaussian fits being biased to a single lengthscale, which would be that determined at step 1. We therefore repeat steps 2 and 3 for the sources identified at step 3 and use a cutoff scale optimized for each fragment. We estimate the scale up to which one fragment is resolved from its surrounding to be twice the first-guess measure of its Gaussian FWHM (see step 3). Therefore, for each of the (here 129) sources, we create a final-guess filtered image and adjust its Gaussian fit parameters.
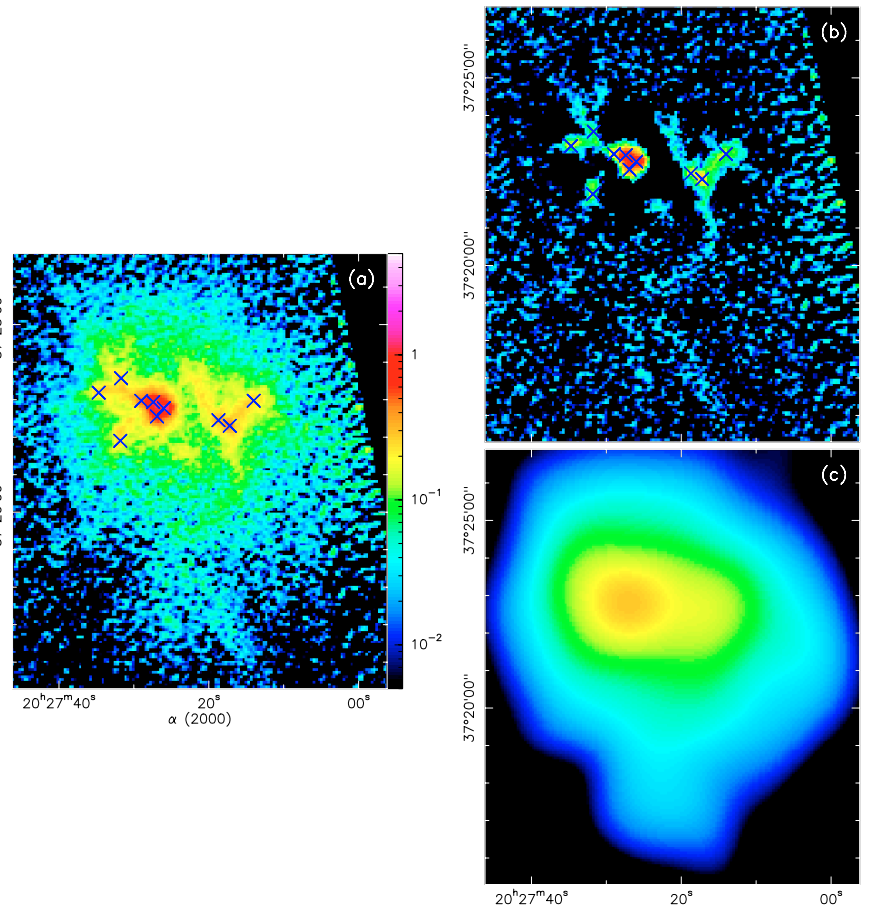

Fig. 11. Separation of cloud structures from the MAMBO-2 map of S106 shown in a) into b) dense cores (i.e. compact structures with size scales smaller than $128^{\prime \prime}$ ) and c) their environment (i.e. cloud structure with a typical scale $>128^{\prime \prime}$ ). Dense cores extracted by the technique described in Appendix B are indicated by crosses.

\footnotetext{
${ }^{8}$ The original image can be expressed as the sum of all wavelet views (also called planes) plus the smoothed image (last plane containing all remaining scales).

${ }^{9}$ Gaussclumps parameters for initial guesses are: $F W H M=1.1 \times$ $H P B W$, aperture $=1.5 \times H P B W$.
} 
F. Motte et al.: High-mass star formation in the Cygnus X complex, Online Material p 4

Table 1. Properties of dense cores (compact cloud fragments) detected in the Cygnus X complex.

\begin{tabular}{|c|c|c|c|c|c|c|c|}
\hline $\begin{array}{l}\text { Fragment } \\
\text { name }\end{array}$ & $\begin{array}{r}S_{(\mathrm{mJy} /}^{\text {peak } a} \\
1.2 \mathrm{~mm} \\
\text { beam })\end{array}$ & $\begin{array}{l}F W H M^{a} \\
(\mathrm{pc} \times \mathrm{pc})\end{array}$ & $S_{1.2 \mathrm{~mm}}^{\mathrm{int}}{ }^{a}$ & $\begin{array}{r}M_{1.2 \mathrm{~mm}^{b}} \\
\left(M_{\odot}\right)\end{array}$ & $\begin{array}{l}\left\langle n_{\mathrm{H}_{2}}\right\rangle^{c} \\
\left(\mathrm{~cm}^{-3}\right)\end{array}$ & $\begin{array}{c}\mathrm{MSX}^{d} \\
21 \mu \mathrm{m} \\
(\mathrm{Jy})\end{array}$ & $\begin{array}{l}\text { Name, comments, and coincidence } \\
\text { with stellar activity signatures }\end{array}$ \\
\hline CygX-North region & & & & & & & \\
\hline N1 J203532.7+421953 & 120 & $0.15 \times 0.09$ & 320 & 17 & $4.7 \times 10^{4}$ & - & \\
\hline N2 J203534.0+422023 & 110 & $0.22 \times 0.07$ & 380 & 20 & $4.0 \times 10^{4}$ & 3.74 & MSX 81.3039+1.0520 \\
\hline N3 J203534.2+422005 & 720 & $0.11 \times 0.09$ & 1580 & 84 & $3.6 \times 10^{5}$ & - & \\
\hline N4 J203558.0+422431 & 90 & $0.06 \times 0.06$ & 120 & $<6$ & $<1.2 \times 10^{5}$ & - & probably not an embedded $\mathrm{YSO}^{e}$ \\
\hline N5 J203606.5+413958 & 160 & $0.12 \times 0.07$ & 350 & 19 & $9.4 \times 10^{4}$ & - & \\
\hline N6 J203608.1+413958 & 260 & $0.23 \times 0.14$ & 1280 & $44-68$ & $3.2-5.0 \times 10^{4}$ & 90.88 & IRAS $20343+4129$ \\
\hline N7 J203615.7+425305 & 80 & $0.12 \times 0.10$ & 190 & 10 & $3.2 \times 10^{4}$ & - & \\
\hline N8 J203638.4+422911 & 170 & $0.17 \times 0.09$ & 500 & 27 & $6.4 \times 10^{4}$ & Abs & \\
\hline N9 J203639.5+425113 & 100 & $0.23 \times 0.14$ & 500 & 26 & $1.7 \times 10^{4}$ & Abs & \\
\hline N10 J203652.2+413623 & 590 & $0.14 \times 0.09$ & 1530 & $<52-81$ & $<1.5-2.4 \times 10^{5}$ & 99.33 & $\begin{array}{l}\text { IRAS } 20350+4126, \mathrm{~cm}^{1} \text { free-free, } \\
\operatorname{maser}^{2}\left(\mathrm{CH}_{3} \mathrm{OH} / \mathrm{OH}\right)\end{array}$ \\
\hline N11 J203656.8+421320 & 180 & $0.11 \times 0.06$ & 340 & 18 & $1.4 \times 10^{5}$ & Abs & \\
\hline N12 J203657.4+421127 & 740 & $0.11 \times 0.09$ & 1630 & 86 & $3.8 \times 10^{5}$ & Abs & \\
\hline N13 J203658.4+421118 & 90 & $0.04 \times 0.03$ & 100 & 5 & $7.1 \times 10^{5}$ & Abs & \\
\hline N14 J203700.9+413457 & 500 & $0.10 \times 0.07$ & 940 & 50 & $3.5 \times 10^{5}$ & 19.71 & IRAS $20352+4124$ \\
\hline N15 J203710.9+413339 & 80 & $0.09 \times 0.03$ & 110 & 6 & $2.1 \times 10^{5}$ & - & \\
\hline N16 J203716.9+421631 & 210 & $0.12 \times 0.12$ & 550 & 29 & $7.5 \times 10^{4}$ & 3.87 & MSX 81.4452+0.7635 \\
\hline N17 J203726.5+413319 & 100 & $0.15 \times 0.03$ & 200 & 10 & $1.6 \times 10^{5}$ & - & \\
\hline N18 J203730.2+421359 & 110 & $0.17 \times 0.13$ & 430 & 23 & $2.7 \times 10^{4}$ & - & \\
\hline N19 J203745.8+424343 & 80 & $0.09 \times 0.03$ & 110 & 6 & $1.9 \times 10^{5}$ & - & \\
\hline N20 J203747.4+423842 & 100 & $0.10 \times 0.03$ & 150 & 8 & $2.1 \times 10^{5}$ & 2.06 & MSX 81.7947+0.9116 \\
\hline N21 J203801.7+423933 & 100 & $0.16 \times 0.14$ & 380 & 20 & $2.5 \times 10^{4}$ & - & \\
\hline N22 J203803.4+423956 & 90 & $0.27 \times 0.11$ & 420 & 22 & $1.9 \times 10^{4}$ & - & \\
\hline N23 J203804.9+423317 & 80 & $0.22 \times 0.06$ & 250 & 13 & $4.0 \times 10^{4}$ & - & \\
\hline N24 J203805.8+423952 & 140 & $0.17 \times 0.17$ & 650 & 34 & $3.0 \times 10^{4}$ & - & \\
\hline N25 J203810.4+423805 & 170 & $0.12 \times 0.11$ & 450 & 24 & $6.5 \times 10^{4}$ & - & \\
\hline N26 J203821.2+421131 & 100 & $0.20 \times 0.10$ & 340 & 18 & $2.6 \times 10^{4}$ & - & \\
\hline N27 J203831.6+423804 & 90 & $0.10 \times 0.10$ & 200 & 11 & $4.1 \times 10^{4}$ & - & \\
\hline N28 J203833.7+423944 & 140 & $0.19 \times 0.11$ & 510 & 27 & $3.9 \times 10^{4}$ & - & \\
\hline N29 J203834.6+420619 & 110 & $0.25 \times 0.13$ & 540 & 29 & $2.0 \times 10^{4}$ & - & \\
\hline N30 J203836.6+423730 & 3650 & $0.15 \times 0.10$ & 10640 & $<361-563$ & $<0.8-1.2 \times 10^{6}$ & 136 & $\begin{array}{l}\text { W75N(B), cm free-free }{ }^{3} \\
\operatorname{maser}^{4}\left(\mathrm{CH}_{3} \mathrm{OH} / \mathrm{H}_{2} \mathrm{O} / \mathrm{OH}\right)\end{array}$ \\
\hline N31 J203836.9+423750 & 120 & $0.03 \times 0.03$ & 130 & 7 & $1.2 \times 10^{6}$ & - & close to $\mathrm{H}$ II region $\mathrm{W} 75 \mathrm{~N}(\mathrm{~A})$ \\
\hline N32 J203838.3+423733 & 540 & $0.16 \times 0.10$ & 1630 & 86 & $1.7 \times 10^{5}$ & - & \\
\hline N33 J203847.0+423800 & 100 & $0.08 \times 0.07$ & 170 & 9 & $8.1 \times 10^{4}$ & - & \\
\hline N34 J203854.3+421917 & 150 & $0.19 \times 0.07$ & 430 & 23 & $6.1 \times 10^{4}$ & - & \\
\hline N35 J203854.4+421855 & 90 & $0.13 \times 0.03$ & 160 & 8 & $1.5 \times 10^{5}$ & - & \\
\hline N36 J203858.5+422330 & 90 & $0.19 \times 0.03$ & 210 & 11 & $1.2 \times 10^{5}$ & - & \\
\hline N37 J203858.6+422435 & 80 & $0.03 \times 0.03$ & 80 & 4 & $8.7 \times 10^{5}$ & - & \\
\hline N38 J203859.4+422223 & 830 & $0.18 \times 0.09$ & 2610 & 138 & $2.7 \times 10^{5}$ & - & DR21(OH)-W \\
\hline N39 J203859.9+422732 & 70 & $0.16 \times 0.08$ & 200 & 10 & $3.0 \times 10^{4}$ & - & \\
\hline N40 J203859.8+422343 & 400 & $0.33 \times 0.08$ & 2000 & 106 & $1.1 \times 10^{5}$ & - & $\mathrm{DR} 21(\mathrm{OH})-\mathrm{N} 2$ \\
\hline N41 J203900.2+422325 & 180 & $0.03 \times 0.03$ & 180 & 10 & $1.9 \times 10^{6}$ & - & $\mathrm{DR} 21(\mathrm{OH})-\mathrm{N} 1$ \\
\hline N42 J203900.3+421907 & 90 & $0.05 \times 0.03$ & 100 & 5 & $4.5 \times 10^{5}$ & - & \\
\hline N43 J203900.6+422435 & 370 & $0.19 \times 0.10$ & 1250 & 66 & $1.1 \times 10^{5}$ & 15.9 & W75S-FIR1, maser $^{5,8}\left(\mathrm{H}_{2} \mathrm{O} / \mathrm{OH}\right)$ \\
\hline N44 J203901.0+422246 & 2970 & $0.14 \times 0.11$ & 8430 & 446 & $1.0 \times 10^{6}$ & 6.78 & $\begin{array}{l}\mathrm{DR} 21(\mathrm{OH}) \\
\operatorname{maser}^{2,8}\left(\mathrm{CH}_{3} \mathrm{OH} / \mathrm{H}_{2} \mathrm{O} / \mathrm{OH}\right)\end{array}$ \\
\hline N45 J203901.1+421814 & 130 & $0.13 \times 0.08$ & 300 & 16 & $6.4 \times 10^{4}$ & Abs & \\
\hline N46 J203901.4+421934 & 4200 & $0.19 \times 0.14$ & 17930 & $<609-949$ & $<5.9-9.1 \times 10^{5}$ & 272 & $\begin{array}{l}\text { DR21, } \mathrm{cm}^{7} \text { free-free, } \\
\text { maser }^{8}\left(\mathrm{H}_{2} \mathrm{O}\right)\end{array}$ \\
\hline N47 J203901.4+421954 & 630 & $0.08 \times 0.03$ & 840 & $<28-44$ & $<1.2-1.9 \times 10^{6}$ & 272 & DR21-D, $\mathrm{cm}^{7}$ free-free \\
\hline N48 J203901.5+422203 & 1160 & $0.17 \times 0.11$ & 3720 & 197 & $3.5 \times 10^{5}$ & - & DR21(OH)-S \\
\hline N49 J203902.0+422700 & 80 & $0.15 \times 0.03$ & 160 & 8 & $1.2 \times 10^{5}$ & - & \\
\hline N50 J203901.9+421835 & 80 & $0.24 \times 0.03$ & 240 & 12 & $1.0 \times 10^{5}$ & - & \\
\hline N51 J203902.4+422459 & 680 & $0.19 \times 0.10$ & 2370 & 125 & $2.0 \times 10^{5}$ & 26.50 & W75S-FIR2 \\
\hline N52 J203903.0+422615 & 180 & $0.16 \times 0.12$ & 630 & 33 & $4.9 \times 10^{4}$ & - & \\
\hline N53 J203903.2+422549 & 590 & $0.14 \times 0.09$ & 1600 & 85 & $2.2 \times 10^{5}$ & - & $\operatorname{maser}^{2}\left(\mathrm{CH}_{3} \mathrm{OH} / \mathrm{H}_{2} \mathrm{O}\right)$ \\
\hline N54 J203903.6+422530 & 150 & $0.18 \times 0.08$ & 450 & 24 & $6.0 \times 10^{4}$ & 10.85 & W75S-FIR3 \\
\hline N55 J203906.1+421819 & 100 & $0.15 \times 0.05$ & 220 & 11 & $8.0 \times 10^{4}$ & Abs & \\
\hline N56 J203916.9+421607 & 270 & $0.12 \times 0.08$ & 600 & 32 & $1.4 \times 10^{5}$ & 3.68 & MSX 81.6632+0.4651 \\
\hline N57 J203919.3+421556 & 130 & $0.09 \times 0.05$ & 210 & 11 & $1.5 \times 10^{5}$ & Abs & ERO1 \\
\hline
\end{tabular}


Table 1. continued.

\begin{tabular}{|c|c|c|c|c|c|c|c|}
\hline $\begin{array}{l}\text { Fragment } \\
\text { name }\end{array}$ & $\begin{array}{c}S_{1.2 \mathrm{~mm}}^{\text {peak }}{ }^{(\mathrm{mJy} /} \\
\text { beam })\end{array}$ & $\begin{array}{l}F W H M^{a} \\
(\mathrm{pc} \times \mathrm{pc})\end{array}$ & $S_{1.2 \mathrm{~mm}}^{\mathrm{int}}{ }_{(\mathrm{mJy})}^{a}$ & $\begin{array}{r}M_{1.2 \mathrm{~mm}^{b}} \\
\left(M_{\odot}\right)\end{array}$ & $\begin{array}{l}\left\langle n_{\mathrm{H}_{2}}\right\rangle^{c} \\
\left(\mathrm{~cm}^{-3}\right)\end{array}$ & $\begin{array}{c}\mathrm{MSX}^{\mathrm{d}} \\
21 \mu \mathrm{m} \\
(\mathrm{Jy})\end{array}$ & $\begin{array}{l}\text { Name, comments, and coincidence } \\
\text { with stellar activity signatures }\end{array}$ \\
\hline N58 J203925.9+412001 & 430 & $0.23 \times 0.19$ & 2730 & $<92-144$ & $<4.2-6.6 \times 10^{4}$ & 634.13 & IRAS $20375+4109, \mathrm{~cm}^{9}$ free-free \\
\hline N59 J203931.2+412003 & 200 & $0.12 \times 0.05$ & 370 & 20 & $2.0 \times 10^{5}$ & - & \\
\hline N60 J203936.2+411937 & 160 & $0.16 \times 0.13$ & 560 & 30 & $4.2 \times 10^{4}$ & - & \\
\hline N61 J203957.6+415911 & 100 & $0.12 \times 0.09$ & 230 & 12 & $4.7 \times 10^{4}$ & 11.63 & MSX 81.5168+0.1926 \\
\hline N62 J204003.6+412756 & 140 & $0.18 \times 0.12$ & 500 & 27 & $3.6 \times 10^{4}$ & - & $\begin{array}{l}\text { close to MSX 81.1109-0.1459 } \\
\text { and MSX 81.1225-0.1343 }\end{array}$ \\
\hline N63 J204005.2+413213 & 740 & $0.08 \times 0.05$ & 1090 & 58 & $1.1 \times 10^{6}$ & Abs & \\
\hline N64 J204027.2+415651 & 100 & $0.12 \times 0.09$ & 220 & 12 & $4.6 \times 10^{4}$ & Abs & \\
\hline N65 J204028.4+415711 & 230 & $0.16 \times 0.12$ & 780 & 41 & $6.4 \times 10^{4}$ & 2.0 & \\
\hline N66 J204029.8+414933 & 110 & $0.15 \times 0.06$ & 250 & 13 & $6.1 \times 10^{4}$ & Abs & \\
\hline N67 J204031.0+414957 & 100 & $0.08 \times 0.05$ & 150 & 8 & $1.4 \times 10^{5}$ & Abs & \\
\hline N68 J204033.5+415903 & 390 & $0.10 \times 0.10$ & 840 & 44 & $2.0 \times 10^{5}$ & Abs & \\
\hline N69 J204033.7+415059 & 110 & $0.47 \times 0.18$ & 1280 & 68 & $1.2 \times 10^{4}$ & - & \\
\hline N70 J204034.3+413845 & 80 & $0.12 \times 0.07$ & 170 & 9 & $5.0 \times 10^{4}$ & Abs & \\
\hline N71 J204038.2+415259 & 80 & $0.21 \times 0.06$ & 230 & 12 & $3.4 \times 10^{4}$ & - & \\
\hline N72 J204045.7+415750 & 100 & $0.28 \times 0.13$ & 540 & 29 & $1.7 \times 10^{4}$ & - & \\
\hline \multicolumn{8}{|l|}{ CygX-South region } \\
\hline S1 J201643.3+392313 & 90 & $0.08 \times 0.05$ & 140 & 8 & $1.1 \times 10^{5}$ & 3.08 & IRAS 20149+3913 \\
\hline S2 J201648.5+392212 & 100 & $0.17 \times 0.06$ & 260 & 14 & $5.4 \times 10^{4}$ & Abs & \\
\hline S3 J201658.6+392106 & 210 & $0.12 \times 0.08$ & 440 & 23 & $1.2 \times 10^{5}$ & 5.27 & IRAS $20151+3911$ \\
\hline S4 J201737.0+392139 & 90 & $0.14 \times 0.10$ & 230 & 12 & $3.1 \times 10^{4}$ & Abs & \\
\hline S5 J201745.3+392041 & 120 & $0.16 \times 0.09$ & 340 & 18 & $4.1 \times 10^{4}$ & - & \\
\hline S6 J202036.5+393821 & 120 & $0.03 \times 0.03$ & 120 & 6 & $1.2 \times 10^{6}$ & - & \\
\hline S7 J202038.0+393820 & 280 & $0.22 \times 0.03$ & 760 & 40 & $3.5 \times 10^{5}$ & - & \\
\hline S8 J202038.8+393751 & 900 & $0.19 \times 0.12$ & 3540 & $<121-188$ & $<1.4-2.1 \times 10^{5}$ & 145.48 & $\begin{array}{l}\text { Mol121S, } \mathrm{cm}^{10} \text { free-free, } \\
\operatorname{maser}^{11}\left(\mathrm{H}_{2} \mathrm{O}\right)\end{array}$ \\
\hline S9 J202039.5+393738 & 120 & $0.03 \times 0.03$ & 130 & 7 & $1.1 \times 10^{6}$ & - & \\
\hline $\mathrm{S} 10 \mathrm{~J} 202044.4+393520$ & 180 & $0.19 \times 0.14$ & 760 & 40 & $4.1 \times 10^{4}$ & - & \\
\hline $\mathrm{S} 11 \mathrm{~J} 202153.7+395933$ & 110 & $0.15 \times 0.11$ & 340 & 18 & $3.6 \times 10^{4}$ & - & \\
\hline $\mathrm{S} 12 \mathrm{~J} 202220.1+395821$ & 130 & $0.16 \times 0.08$ & 350 & 18 & $5.5 \times 10^{4}$ & - & close to IRAS $20205+3948$ \\
\hline S13 J202632.4+395721 & 160 & $0.15 \times 0.11$ & 500 & 27 & $5.0 \times 10^{4}$ & 4.84 & MSX $78.3762+1.0191$ \\
\hline S14 J202634.8+395725 & 200 & $0.09 \times 0.05$ & 330 & 17 & $2.4 \times 10^{5}$ & Abs & \\
\hline $\mathrm{S} 15 \mathrm{~J} 202714.0+372258$ & 120 & $0.29 \times 0.12$ & 630 & 34 & $2.2 \times 10^{4}$ & Abs & \\
\hline S16 J202717.2+372218 & 260 & $0.11 \times 0.05$ & 460 & 24 & $3.0 \times 10^{5}$ & - & \\
\hline S17 J202718.7+372227 & 130 & $0.07 \times 0.06$ & 210 & 11 & $1.4 \times 10^{5}$ & - & \\
\hline S18 J202726.0+372246 & 960 & $0.21 \times 0.13$ & 4270 & $<145-226$ & $<1.2-2.0 \times 10^{5}$ & 413.63 & $\begin{array}{l}\text { S106-IR, } \mathrm{cm}^{12} \text { free-free, } \\
\operatorname{maser}^{8}\left(\mathrm{H}_{2} \mathrm{O}\right)\end{array}$ \\
\hline S19 J202726.9+372233 & 310 & $0.08 \times 0.03$ & 420 & $<14-22$ & $<6.1-9.5 \times 10^{5}$ & 413.63 & part of S106-IR, $\mathrm{cm}^{12}$ free-free \\
\hline S20 J202727.4+372256 & 510 & $0.11 \times 0.11$ & 1300 & $<44-69$ & $<1.2-2.0 \times 10^{5}$ & 413.63 & part of S106-IR, $\mathrm{cm}^{1}$ free-free \\
\hline $\mathrm{S} 21 \mathrm{~J} 202729.0+372258$ & 120 & $0.03 \times 0.03$ & 130 & 7 & $1.2 \times 10^{6}$ & - & \\
\hline S22 J202731.7+372334 & 80 & $0.13 \times 0.03$ & 150 & 8 & $1.5 \times 10^{5}$ & - & \\
\hline S23 J202731.8+372154 & 110 & $0.24 \times 0.03$ & 310 & 16 & $1.2 \times 10^{5}$ & - & \\
\hline $\mathrm{S} 24 \mathrm{~J} 202734.7+372311$ & 140 & $0.18 \times 0.09$ & 420 & 22 & $4.9 \times 10^{4}$ & - & \\
\hline $\mathrm{S} 25 \mathrm{~J} 202923.6+401110$ & 120 & $0.05 \times 0.03$ & 140 & 7 & $6.1 \times 10^{5}$ & - & \\
\hline S26 J202924.8+401118 & 880 & $0.14 \times 0.10$ & 2340 & $<80-124$ & $<2.1-3.4 \times 10^{5}$ & 1023.40 & $\begin{array}{l}\text { AFGL } 2591, \mathrm{~cm}^{13} \text { free-free, } \\
\operatorname{maser}^{8}\left(\mathrm{H}_{2} \mathrm{O} / \mathrm{OH}\right)\end{array}$ \\
\hline S27 J202931.8+390113 & 130 & $0.19 \times 0.09$ & 420 & $<14-22$ & $<2.7-4.2 \times 10^{4}$ & 23.55 & $\begin{array}{l}\text { MSX 77.9550+0.0058, } \\
\mathrm{cm}^{9} \text { free-free }\end{array}$ \\
\hline S28 J202936.8+390112 & 120 & $0.12 \times 0.08$ & 270 & $<9-14$ & $<3.6-5.6 \times 10^{4}$ & 204.20 & IRAS $20277+3851, \mathrm{~cm}^{1}$ free-free \\
\hline S29 J202958.3+401558 & 160 & $0.23 \times 0.12$ & 710 & 37 & $3.4 \times 10^{4}$ & - & \\
\hline S30 J203112.6+400316 & 360 & $0.16 \times 0.16$ & 1560 & 82 & $7.7 \times 10^{4}$ & - & close to IRAS $20293+3952$ \\
\hline S31 J203114.2+400305 & 110 & $0.13 \times 0.07$ & 220 & 12 & $6.5 \times 10^{4}$ & - & \\
\hline S32 J203120.3+385716 & 250 & $0.21 \times 0.11$ & 1010 & 54 & $6.0 \times 10^{4}$ & - & \\
\hline S33 J203144.5+385639 & 80 & $0.14 \times 0.11$ & 230 & 12 & $2.7 \times 10^{4}$ & - & \\
\hline S34 J203157.8+401830 & 150 & $0.20 \times 0.12$ & 610 & 32 & $3.4 \times 10^{4}$ & - & in IRDC G79.3-P3 \\
\hline S35 J203158.8+385836 & 200 & $0.12 \times 0.06$ & 410 & 22 & $1.4 \times 10^{5}$ & 5.00 & \\
\hline S36 J203220.8+401949 & 80 & $0.07 \times 0.03$ & 100 & 5 & $2.5 \times 10^{5}$ & - & in IRDC G79.34+0.33 \\
\hline S37 J203222.0+402012 & 240 & $0.18 \times 0.12$ & 860 & 45 & $6.4 \times 10^{4}$ & 4.44 & IRAS $20305+4010$ \\
\hline S38 J203222.4+401919 & 90 & $0.23 \times 0.12$ & 410 & 22 & $2.0 \times 10^{4}$ & - & in IRDC G79.34+0.33 \\
\hline
\end{tabular}


Table 1. continued.

\begin{tabular}{|c|c|c|c|c|c|c|c|}
\hline $\begin{array}{l}\text { Fragment } \\
\text { name }\end{array}$ & $\begin{array}{r}S_{1.2 \mathrm{~mm}}^{\text {peak }}{ }^{a} \\
\text { beam })\end{array}$ & $\begin{array}{l}F W H M^{a} \\
(\mathrm{pc} \times \mathrm{pc})\end{array}$ & $S_{1.2 \mathrm{~mm}}^{\mathrm{int}}{ }_{(\mathrm{mJy})}^{a}$ & $\begin{array}{r}M_{1.2} \mathrm{~mm}^{b} \\
\left(M_{\odot}\right)\end{array}$ & $\begin{array}{l}\left\langle n_{\mathrm{H}_{2}}\right\rangle^{c} \\
\left(\mathrm{~cm}^{-3}\right)\end{array}$ & $\begin{array}{c}\mathrm{MSX}^{d} \\
21 \mu \mathrm{m} \\
(\mathrm{Jy})\end{array}$ & $\begin{array}{l}\text { Name, comments, and coincidence } \\
\text { with stellar activity signatures }\end{array}$ \\
\hline S39 J203229.0+401600 & 170 & $0.14 \times 0.09$ & 440 & $<15-23$ & $<4.2-6.6 \times 10^{4}$ & 37.71 & $\begin{array}{l}\text { MSX 79.2963+0.2835, } \\
\mathrm{cm}^{1} \text { free-free }\end{array}$ \\
\hline S40 J203232.2+401625 & 80 & $0.16 \times 0.10$ & 240 & $<8-13$ & $<1.6-2.6 \times 10^{4}$ & 73.03 & MSX $79.3070+0.2768, \mathrm{~cm}^{9}$ free-free \\
\hline S41 J203233.9+401653 & 220 & $0.19 \times 0.11$ & 800 & $27-42$ & $3.7-5.9 \times 10^{4}$ & 49.4 & part of IRAS $20306+4005$ \\
\hline S42 J203239.9+384603 & 90 & $0.18 \times 0.13$ & 340 & 18 & $2.0 \times 10^{4}$ & - & \\
\hline S43 J203240.8+384631 & 140 & $0.23 \times 0.13$ & 650 & 35 & $2.9 \times 10^{4}$ & Abs & \\
\hline \multicolumn{8}{|l|}{ CygX-NW region } \\
\hline NW1 J201938.8+405638 & 280 & $0.17 \times 0.15$ & 1110 & $<38-5-9$ & $<4.2-6.6 \times 10^{4}$ & 400.66 & IRAS $20178+4046, \mathrm{~cm}^{1}$ free-free \\
\hline NW2 J201940.5+405704 & 200 & $0.23 \times 0.07$ & 680 & 36 & $7.7 \times 10^{4}$ & - & \\
\hline NW3 J202029.9+412144 & 80 & $0.15 \times 0.07$ & 190 & 10 & $4.2 \times 10^{4}$ & - & \\
\hline NW4 J202030.0+412207 & 130 & $0.07 \times 0.05$ & 180 & 10 & $2.2 \times 10^{5}$ & - & \\
\hline NW5 J202030.7+412125 & 420 & $0.07 \times 0.06$ & 640 & $22-34$ & $3.2-5.0 \times 10^{5}$ & 85.85 & $\mathrm{EM}^{*}$ LkHA $225 \mathrm{~S}, \operatorname{maser}^{14}\left(\mathrm{H}_{2} \mathrm{O}\right)$ \\
\hline NW6 J202032.1+412354 & 130 & $0.15 \times 0.03$ & 250 & 13 & $2.0 \times 10^{5}$ & - & \\
\hline NW7 J202032.2+412123 & 80 & $0.07 \times 0.03$ & 100 & 6 & $2.5 \times 10^{5}$ & - & \\
\hline NW8 J202323.0+411749 & 90 & $0.10 \times 0.04$ & 150 & 8 & $1.2 \times 10^{5}$ & - & \\
\hline NW9 J202324.0+411739 & 230 & $0.10 \times 0.04$ & 370 & 19 & $3.0 \times 10^{5}$ & 27.47 & IRAS 20216+4107 \\
\hline NW10 J202343.6+411657 & 160 & $0.20 \times 0.08$ & 520 & 27 & $5.7 \times 10^{4}$ & - & \\
\hline NW11 J202413.0+411700 & 110 & $0.13 \times 0.09$ & 290 & 15 & $4.7 \times 10^{4}$ & - & \\
\hline NW12 J202414.3+421143 & 140 & $0.06 \times 0.06$ & 200 & 11 & $2.1 \times 10^{5}$ & Abs & \\
\hline NW13 J202419.5+421545 & 100 & $0.17 \times 0.09$ & 290 & 15 & $3.5 \times 10^{4}$ & - & \\
\hline NW14 J202431.7+420423 & 420 & $0.12 \times 0.09$ & 940 & $32-50$ & $1.2-2.0 \times 10^{5}$ & 15.58 & $\begin{array}{l}\text { Mol124 = IRAS 20227+4154, } \\
\text { maser }^{11}\left(\mathrm{H}_{2} \mathrm{O}\right)\end{array}$ \\
\hline
\end{tabular}

Notes:

a Peak flux, deconvolved FWHM size, and integrated flux derived from a 2D-Gaussian fit to the $1.2 \mathrm{~mm}$ map after background subtraction (cf. Sect. 3.2 and Appendix B). An upper limit FWHM of $0.03 \mathrm{pc}$ was assumed for unresolved fragments.

${ }^{b}$ Mass derived from Col. 5 using Eq. (1) and assuming $\kappa_{1.2 \mathrm{~mm}}=0.01 \mathrm{~cm}^{2} \mathrm{~g}^{-1}$ and $T_{\text {dust }}=20 \mathrm{~K}$. A second estimate with $T_{\text {dust }}=40 \mathrm{~K}$ is made for $\mathrm{H}$ II regions and the brightest infrared protostars of Cygnus X.

$c$ Volume-averaged density derived from Cols. 4 and 6 using Eq. (2).

$d$ The $21 \mu$ m fluxes are those given in the MSX point source catalog (MSX C6), except for CygX-N30, N43, N46, N47, N65 and S41, for which an aperture flux is measured on the MSX map. The MSX flux of two extended H II regions is equally distributed between CygX-N46 and N47, and between CygX-S18, S19, S20. All fragments detected at $21 \mu \mathrm{m}$ are also detected at $8 \mu \mathrm{m}$ with the exception of CygX-N30, N46, N47, and N65. "Abs" means that sources are seen in absorption at $8 \mu \mathrm{m}$.

$e$ Observations of high-density tracers toward CygX-N4 suggest that this $1.2 \mathrm{~mm}$ source does not correspond to a dense cloud fragment. Its nature is yet unclear but it is probably not a site for future or ongoing star formation.

References: (1) Kurtz et al. (1994); (2) Pestalozzi et al. (2005); (3) Haschick et al. (1981); (4) Minier et al. (2001); (5) Argon et al. (2000); (7) Harris (1973); (8) Braz \& Epchtein (1983); (9) Zoonematkermani et al. (1990); (10) Molinari et al. (1998b); (11) Palla et al. (1991); (12) Pipher et al. (1976); (13) Trinidad et al. (2003); (14) Palla et al. (1995). 
F. Motte et al.: High-mass star formation in the Cygnus X complex, Online Material $p 7$

Table 2. Properties of clumps (large-scale cloud structures) detected in the Cygnus X complex.

\begin{tabular}{|c|c|c|c|c|c|c|}
\hline & $\begin{array}{c}\text { Structure } \\
\text { name }\end{array}$ & $\begin{array}{l}F W H M^{a} \\
(\mathrm{pc} \times \mathrm{pc})\end{array}$ & $S_{1.2 \mathrm{~mm}}^{\mathrm{int}}{ }_{(\mathrm{mJy})}^{a}$ & $\begin{array}{r}M_{1.2 \mathrm{~mm}^{b}} \\
\left(M_{\odot}\right)\end{array}$ & $\begin{array}{l}\left\langle n_{\mathrm{H}_{2}}\right\rangle^{c} \\
\left(\mathrm{~cm}^{-3}\right)\end{array}$ & Comments \\
\hline & \multicolumn{6}{|l|}{ CygX-North region } \\
\hline Cl-N1 & $\mathrm{J} 203534.1+422004$ & $0.48 \times 0.31$ & 8470 & 665 & $5.0 \times 10^{4}$ & contains CygX-N1, N2, N3 \\
\hline $\mathrm{Cl}-\mathrm{N} 2$ & $\mathrm{~J} 203605.0+420851$ & $1.46 \times 0.94$ & 15150 & 1189 & $3.1 \times 10^{3}$ & Starless? \\
\hline $\mathrm{Cl}-\mathrm{N} 3$ & $\mathrm{~J} 203607.3+414001$ & $0.64 \times 0.38$ & 7010 & 550 & $1.9 \times 10^{4}$ & contains CygX-N5, N6 \\
\hline $\mathrm{Cl}-\mathrm{N} 4$ & $\mathrm{~J} 203651.5+413625$ & $0.66 \times 0.65$ & 14780 & 1161 & $1.7 \times 10^{4}$ & contains CygX-N10 \\
\hline $\mathrm{Cl}-\mathrm{N} 5$ & $\mathrm{~J} 203657.9+421132$ & $0.58 \times 0.49$ & 11690 & 917 & $2.5 \times 10^{4}$ & contains CygX-N12, N13 \\
\hline $\mathrm{Cl}-\mathrm{N} 6$ & $\mathrm{~J} 203701.9+413453$ & $0.81 \times 0.64$ & 13580 & 1066 & $1.2 \times 10^{4}$ & contains CygX-N14 \\
\hline Cl-N7 & $\mathrm{J} 203711.9+413338$ & $1.45 \times 0.90$ & 16370 & 1285 & $3.6 \times 10^{3}$ & contains CygX-N15 \\
\hline $\mathrm{Cl}-\mathrm{N} 8$ & $\mathrm{~J} 203728.4+421630$ & $1.80 \times 1.01$ & 17060 & 1339 & $2.3 \times 10^{3}$ & \\
\hline $\mathrm{Cl}-\mathrm{N} 9$ & $\mathrm{~J} 203729.8+421409$ & $1.32 \times 0.73$ & 14350 & 1127 & $4.9 \times 10^{3}$ & contains CygX-N18 \\
\hline $\mathrm{Cl}-\mathrm{N} 10$ & $\mathrm{~J} 203805.3+423937$ & $0.87 \times 0.51$ & 10000 & 785 & $1.1 \times 10^{4}$ & Starless? contains CygX-N21, N22, N24 \\
\hline $\mathrm{Cl}-\mathrm{N} 11$ & $\mathrm{~J} 203833.2+420619$ & $1.25 \times 0.74$ & 14880 & 1168 & $5.5 \times 10^{3}$ & Starless? contains CygX-N29 \\
\hline $\mathrm{Cl}-\mathrm{N} 12$ & J203836.3+423937 & $1.30 \times 0.56$ & 10240 & 804 & $5.5 \times 10^{3}$ & contains CygX-N28 \\
\hline $\mathrm{Cl}-\mathrm{N} 13$ & $\mathrm{~J} 203836.8+423734$ & $0.48 \times 0.40$ & 46930 & 3684 & $1.9 \times 10^{5}$ & contains CygX-N30, N31, N32 \\
\hline $\mathrm{Cl}-\mathrm{N} 14$ & $\mathrm{~J} 203900.5+422238$ & $0.88 \times 0.44$ & 92460 & 7259 & $1.3 \times 10^{5}$ & contains CygX-N36, N38, N41, N44, N48 \\
\hline $\mathrm{Cl}-\mathrm{N} 15$ & $\mathrm{~J} 203901.3+421935$ & $0.56 \times 0.39$ & 70210 & 5513 & $2.3 \times 10^{5}$ & contains CygX-N42, N46, N47 \\
\hline Cl-N16 & $\mathrm{J} 203901.7+422507$ & $0.91 \times 0.60$ & 47360 & 3718 & $3.8 \times 10^{4}$ & contains CygX-N37, N43, N51, N53, N54 \\
\hline Cl-N17 & J203927.2+412009 & $0.78 \times 0.59$ & 15980 & 1254 & $1.7 \times 10^{4}$ & contains CygX-N58, N59 \\
\hline Cl-N18 & J203936.4+412001 & $0.91 \times 0.36$ & 5710 & 449 & $1.0 \times 10^{4}$ & Starless? contains CygX-N60 \\
\hline Cl-N19 & J203955.4+420020 & $1.79 \times 1.10$ & 18110 & 1422 & $2.2 \times 10^{3}$ & contains CygX-N61 \\
\hline $\mathrm{Cl}-\mathrm{N} 20$ & J204003.6+412814 & $1.03 \times 0.77$ & 14080 & 1105 & $6.6 \times 10^{3}$ & contains CygX-N62 \\
\hline $\mathrm{Cl}-\mathrm{N} 21$ & $\mathrm{~J} 204005.1+413210$ & $0.31 \times 0.20$ & 3350 & 263 & $7.0 \times 10^{4}$ & contains CygX-N63 \\
\hline Cl-N22 & $\mathrm{J} 204027.8+415707$ & $0.68 \times 0.37$ & 5200 & 408 & $1.4 \times 10^{4}$ & contains CygX-N64, N65 \\
\hline \multirow[t]{2}{*}{ Cl-N23 } & $\mathrm{J} 204033.3+415045$ & $0.80 \times 0.57$ & 8700 & 683 & $9.4 \times 10^{3}$ & Starless? contains CygX-N67, N69 \\
\hline & \multicolumn{6}{|l|}{ CygX-South region } \\
\hline $\mathrm{Cl}-\mathrm{S} 1$ & $\mathrm{~J} 202038.5+393753$ & $0.51 \times 0.34$ & 13920 & 1093 & $6.3 \times 10^{4}$ & contains CygX-S6, S7, S8, S9 \\
\hline $\mathrm{Cl}-\mathrm{S} 2$ & $\mathrm{~J} 202044.8+393525$ & $0.67 \times 0.45$ & 4870 & 382 & $9.4 \times 10^{3}$ & contains CygX-S10 \\
\hline $\mathrm{Cl}-\mathrm{S} 3$ & J202716.1+372231 & $1.23 \times 0.69$ & 26640 & 2092 & $1.1 \times 10^{4}$ & contains CygX-S15, S16, S17 \\
\hline $\mathrm{Cl}-\mathrm{S} 4$ & $\mathrm{~J} 202726.9+372248$ & $0.73 \times 0.53$ & 33420 & 2624 & $4.6 \times 10^{4}$ & contains CygX-S18, S19, S20, S21 \\
\hline Cl-S5 & $\mathrm{J} 202924.9+401116$ & $0.42 \times 0.38$ & 10090 & 792 & $5.2 \times 10^{4}$ & contains CygX-S25, S26 \\
\hline Cl-S6 & J202959.4+401555 & $1.25 \times 0.60$ & 11560 & 908 & $5.9 \times 10^{3}$ & Starless? contains CygX-S29 \\
\hline $\mathrm{Cl}-\mathrm{S} 7$ & $\mathrm{~J} 203112.1+400314$ & $0.62 \times 0.49$ & 10710 & 841 & $2.1 \times 10^{4}$ & contains CygX-S30, S31 \\
\hline $\mathrm{Cl}-\mathrm{S} 8$ & J203119.2+385731 & $0.86 \times 0.56$ & 8740 & 686 & $8.6 \times 10^{3}$ & contains CygX-S32 \\
\hline $\mathrm{Cl}-\mathrm{S} 9$ & $\mathrm{~J} 203156.7+401833$ & $1.39 \times 0.60$ & 12140 & 953 & $5.2 \times 10^{3}$ & Starless? IRDC G79.33P3, contains CygX-S34 \\
\hline Cl-S10 & $\mathrm{J} 203221.9+401955$ & $0.92 \times 0.79$ & 17490 & 1373 & $9.3 \times 10^{3}$ & contains CygX-S36, S37, S38 \\
\hline Cl-S11 & $\mathrm{J} 203232.3+401635$ & $0.99 \times 0.67$ & 14010 & 1100 & $8.5 \times 10^{3}$ & contains CygX-S39, S40, S41 \\
\hline $\mathrm{Cl}-\mathrm{S} 12$ & J203240.7+384614 & $0.67 \times 0.52$ & 6480 & 509 & $1.0 \times 10^{4}$ & Starless? contains CygX-S42, S43 \\
\hline \multicolumn{7}{|c|}{ CygX-NW region } \\
\hline Cl-NW1 & $\mathrm{J} 201939.0+405651$ & $0.73 \times 0.64$ & 14190 & 1114 & $1.5 \times 10^{4}$ & contains CygX-NW1, NW2 \\
\hline Cl-NW2 & $\mathrm{J} 202030.5+412132$ & $0.64 \times 0.41$ & 6890 & 541 & $1.7 \times 10^{4}$ & contains CygX-NW3, NW4, NW5, NW7 \\
\hline Cl-NW3 & $\mathrm{J} 202344.5+411701$ & $0.97 \times 0.71$ & 11480 & 901 & $6.5 \times 10^{3}$ & Starless?, contains CygX-NW10 \\
\hline Cl-NW4 & $\mathrm{J} 202420.3+421527$ & $0.96 \times 0.66$ & 8160 & 640 & $5.3 \times 10^{3}$ & Starless?, contains CygX-NW13 \\
\hline Cl-NW5 & $\mathrm{J} 202431.7+420419$ & $0.73 \times 0.60$ & 8670 & 681 & $9.8 \times 10^{3}$ & contains CygX-NW14 \\
\hline
\end{tabular}
Notes:

a Deconvolved $F W H M$ size and integrated flux derived from a 2D-Gaussian fit to the $1.2 \mathrm{~mm}$ map smoothed to a $55^{\prime \prime}$ beam.

${ }^{b}$ Mass derived from Col. 4 using Eq. (1) and assuming $\kappa 1.2 \mathrm{~mm}=0.01 \mathrm{~cm}^{2} \mathrm{~g}^{-1}$ and $T_{\text {dust }}=15 \mathrm{~K}$.

$c$ Volume-averaged density derived from Col. 3 and Col. 5 using Eq. (2). 
F. Motte et al.: High-mass star formation in the Cygnus X complex, Online Material $p 8$
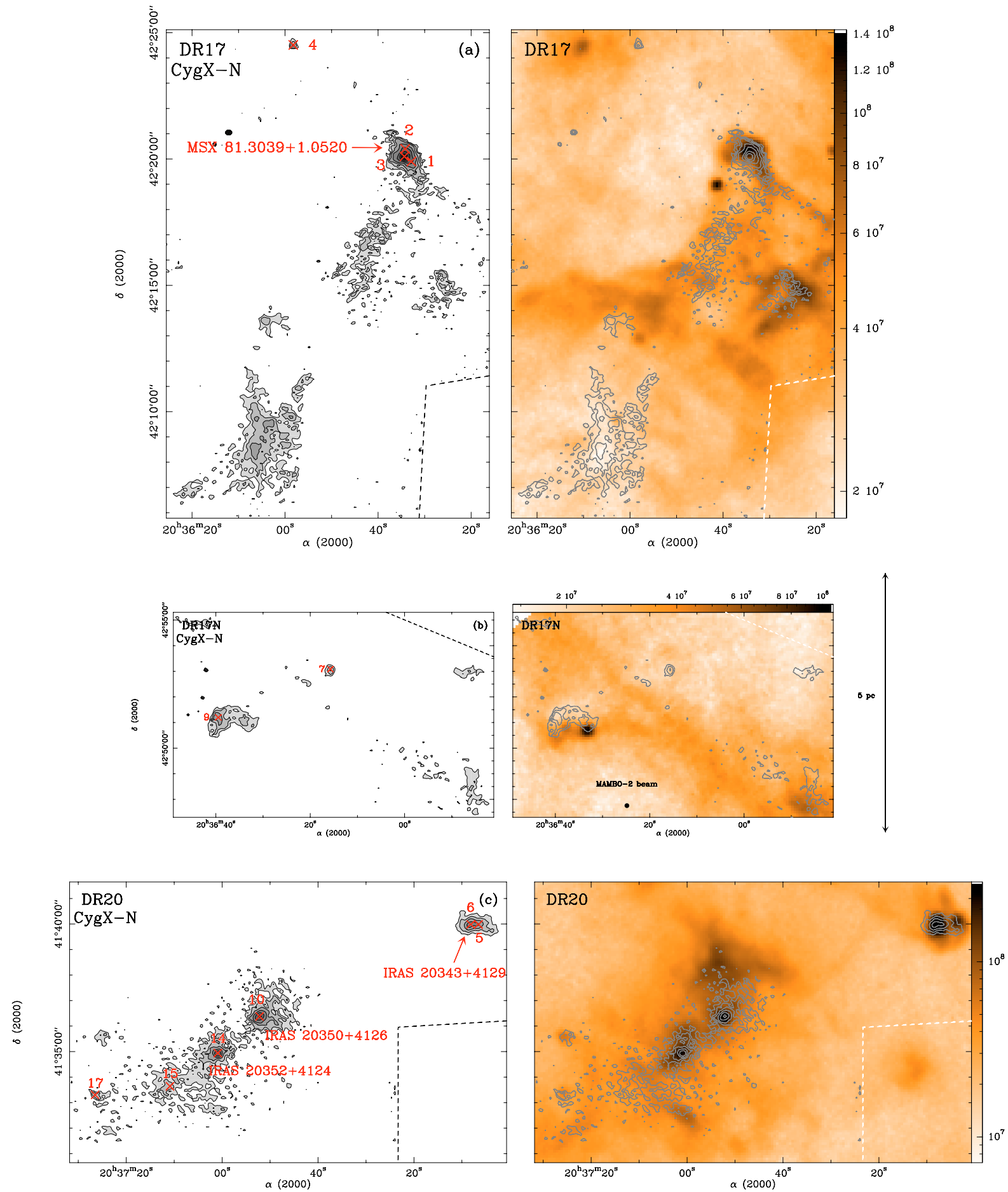

Fig. 12. MAMBO maps of CygX-North (left: gray-scale and contours, right: contours overlaid on $8 \mu \mathrm{m}$ images obtained by $M S X$ and converted to Jy sr ${ }^{-1}$ ) extracted from Fig. 2a. Regions shown are south and north of DR17 a), b) and around DR20 c). The $1.2 \mathrm{~mm}$ and $8 \mu \mathrm{m}$ images have $11^{\prime \prime}$ and $20^{\prime \prime}$ angular resolutions, respectively. The compact cloud fragments discovered in MAMBO images (see Table 1) are labeled and marked by crosses in the gray-scale plot. The infrared sources which coincide with a MAMBO cloud fragment are also indicated. Contour levels are logarithmic and go from 40 to $800 \mathrm{mJy} \mathrm{beam}^{-1}$ in a) and b), and from 75 to $800 \mathrm{mJy} \mathrm{beam}^{-1}$ in c). 
F. Motte et al.: High-mass star formation in the Cygnus X complex, Online Material $p 9$
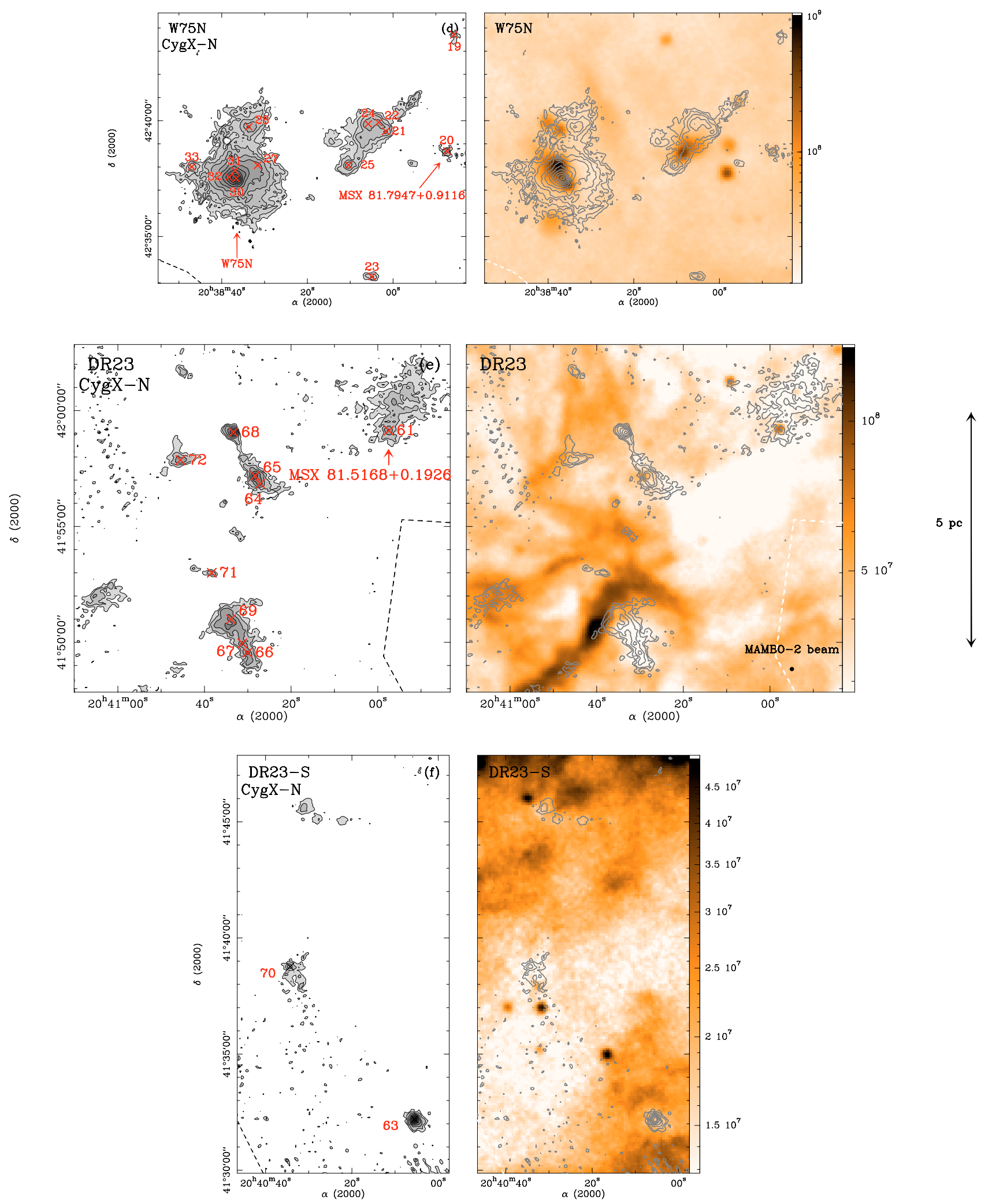

Fig. 12. continued. Same caption for the region around $W 75 \mathrm{~N}$ d) and DR23 e), and south of DR23 f). Contour levels are logarithmic and go from 40 to $4800 \mathrm{mJy}^{-1}$ beam $^{-1}$ in d), and from 40 to $800 \mathrm{mJy} \mathrm{beam}^{-1}$ in e) and f). 
F. Motte et al.: High-mass star formation in the Cygnus X complex, Online Material p 10
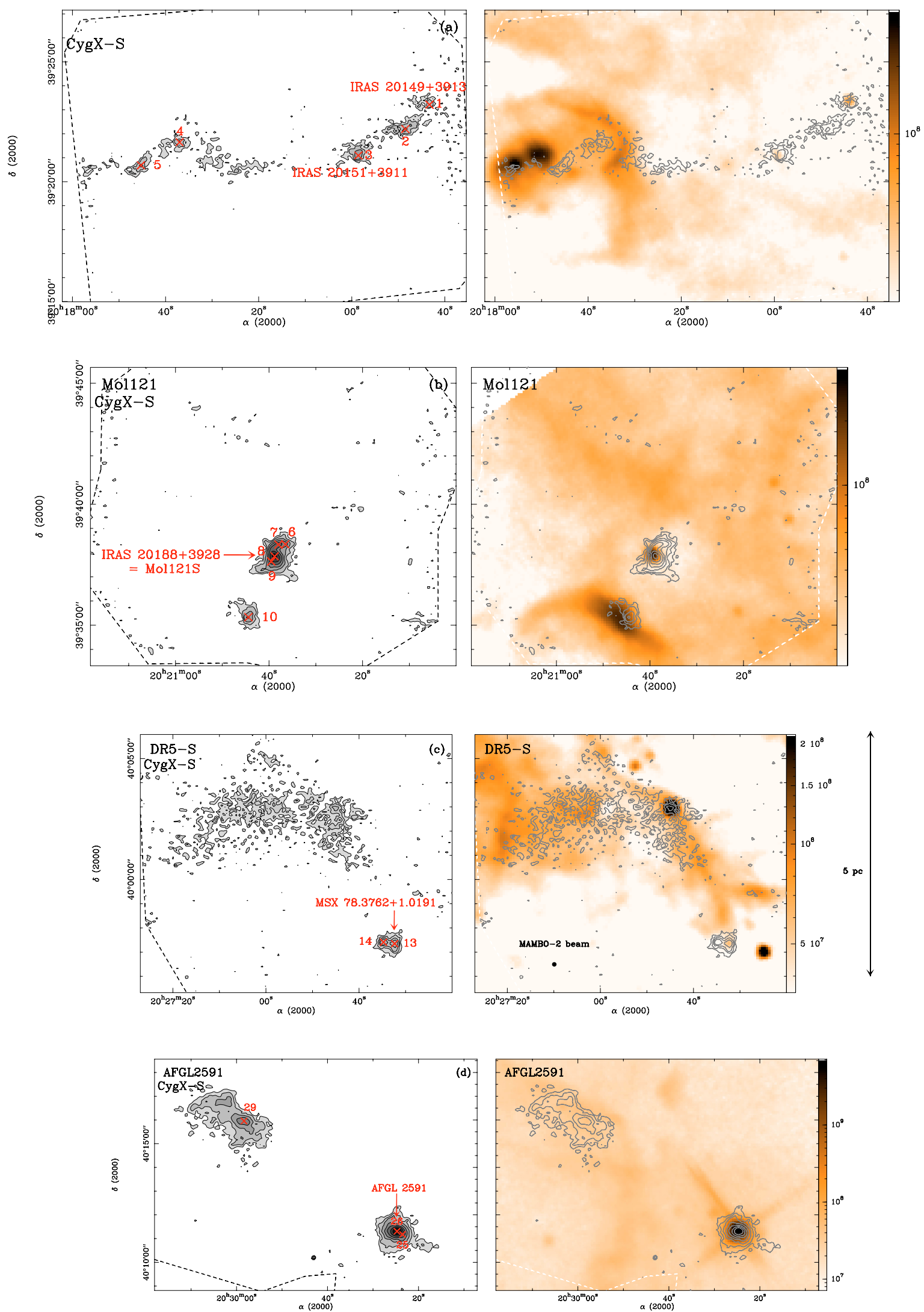

Fig. 13. Same convention as Fig. 12 for MAMBO maps of CygX-South extracted from Fig. 2b. Regions shown are the most western part of CygXSouth a), around Mol121 b), around AFGL 2591 c), and south of DR5 d). Contour levels are logarithmic and go from 60 to $180 \mathrm{mJy} \mathrm{beam}^{-1}$ in a), from 60 to $2000 \mathrm{mJy}_{\text {beam }}{ }^{-1}$ in b), from 40 to $200 \mathrm{mJy} \mathrm{beam}^{-1}$ in c), and from 40 to $1000 \mathrm{mJy} \mathrm{beam}^{-1}$ in d). 
F. Motte et al.: High-mass star formation in the Cygnus X complex, Online Material $p 11$
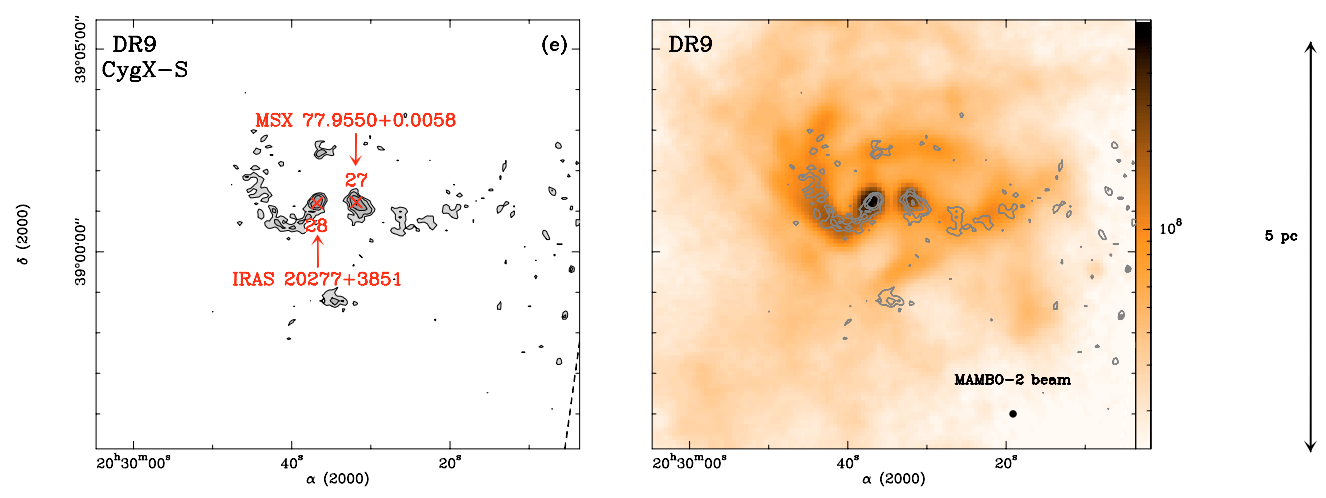

$10^{8}$

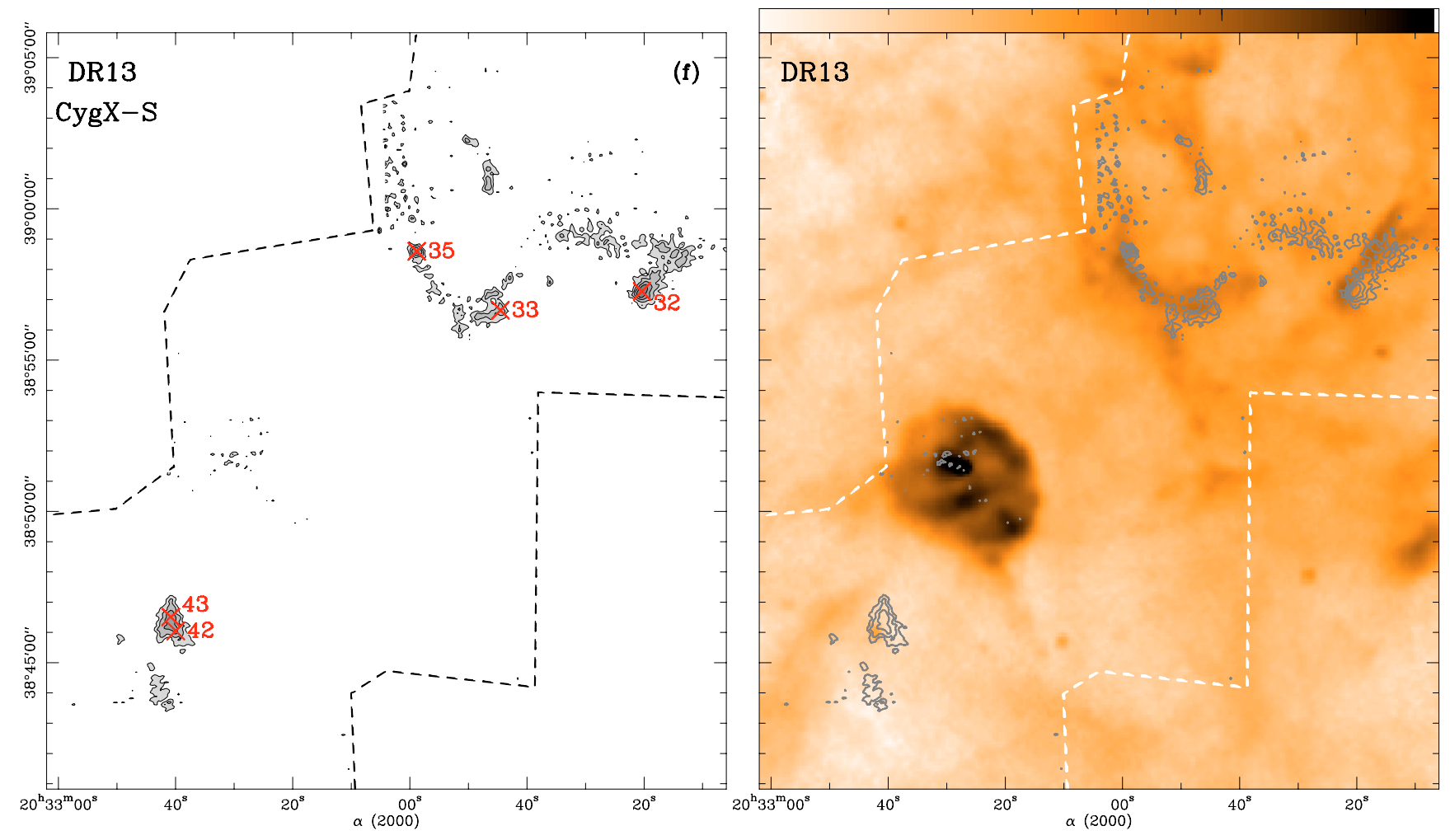

Fig. 13. continued. Same caption for the region around DR9 e) and DR13 f). Contour levels are logarithmic and go from 40 to $200 \mathrm{mJy}^{-1} \mathrm{beam}{ }^{-1}$ in e), and from 60 to $300 \mathrm{mJy} \mathrm{beam}^{-1}$ in f). 
F. Motte et al.: High-mass star formation in the Cygnus X complex, Online Material p 12
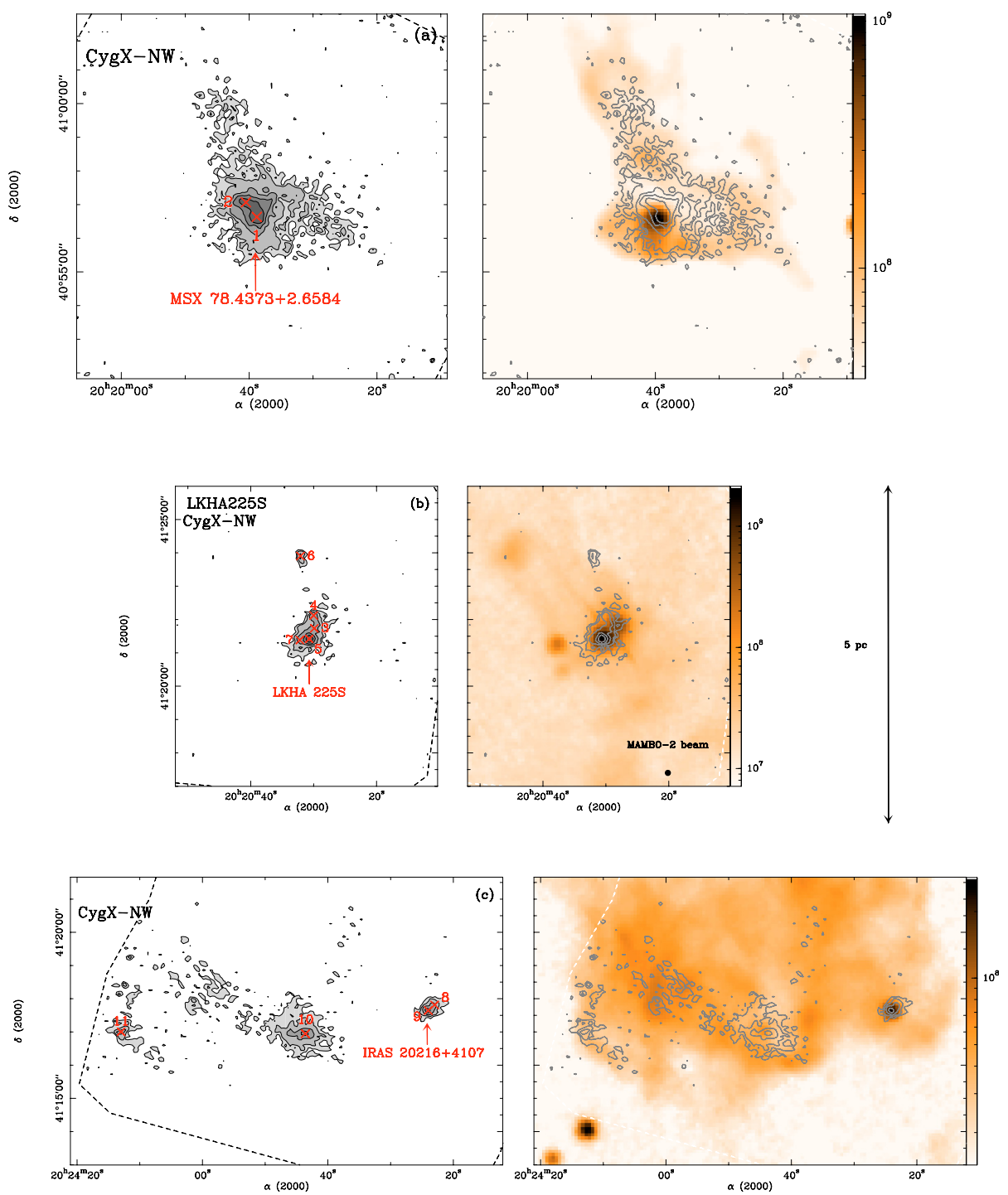

Fig. 14. Same convention as Fig. 12 for MAMBO maps of CygX-NW extracted from Fig. 2c. Regions shown are those of Fig. 2c4 a), of Fig. 2c3 b), and of Fig. 2c2 c). Contour levels are logarithmic and go from 40 to $300 \mathrm{mJy}^{-1}$ beam $^{-1}$ in a), from 60 to $400 \mathrm{mJy} \mathrm{beam}^{-1}$ in b), and from 60 to

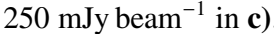


F. Motte et al.: High-mass star formation in the Cygnus X complex, Online Material $p 13$
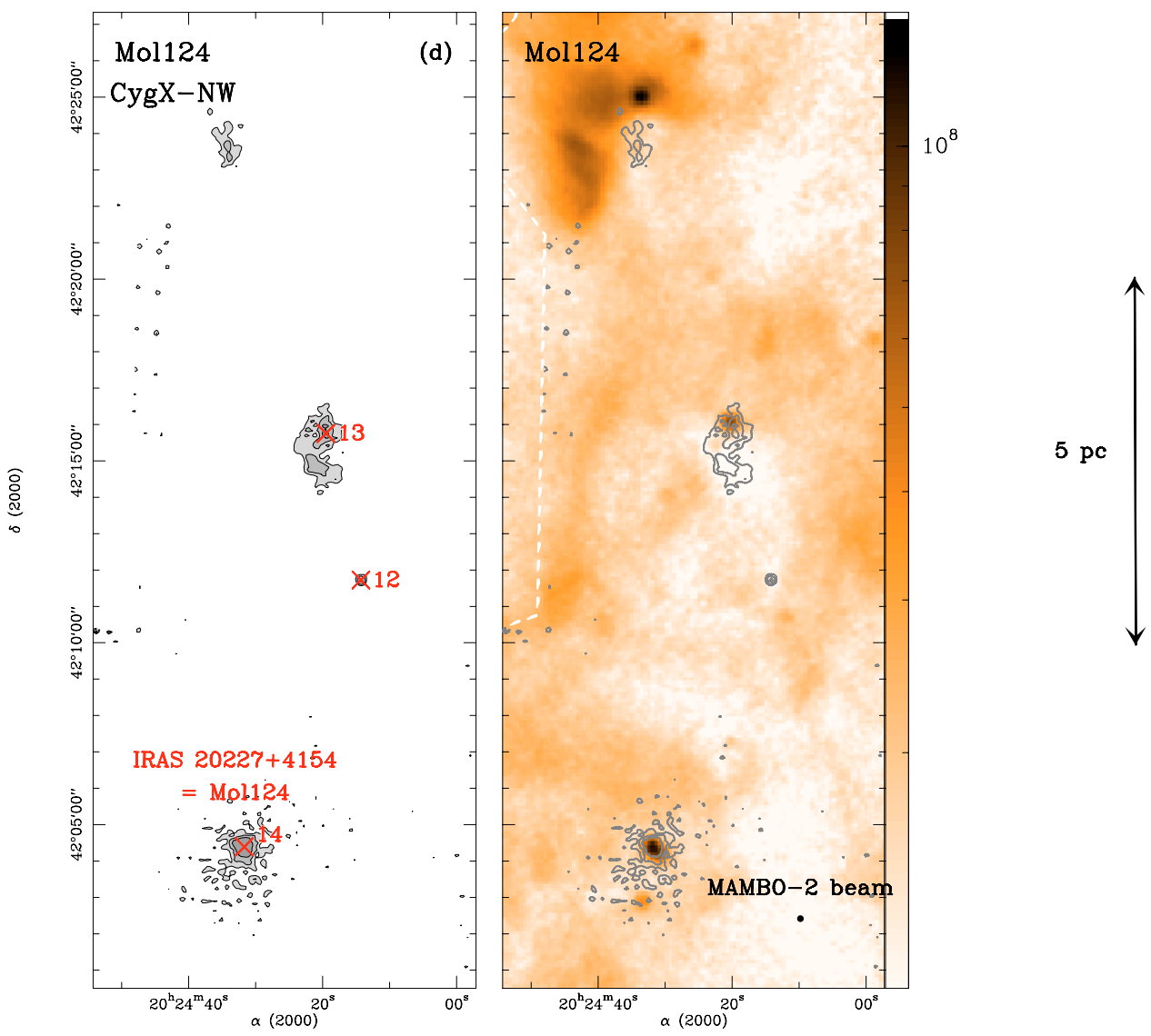

Fig. 14. continued. Same caption for the region around Mol124 d). Contour levels are logarithmic and go from 60 to $180 \mathrm{mJy} \mathrm{beam}^{-1}$ in d).

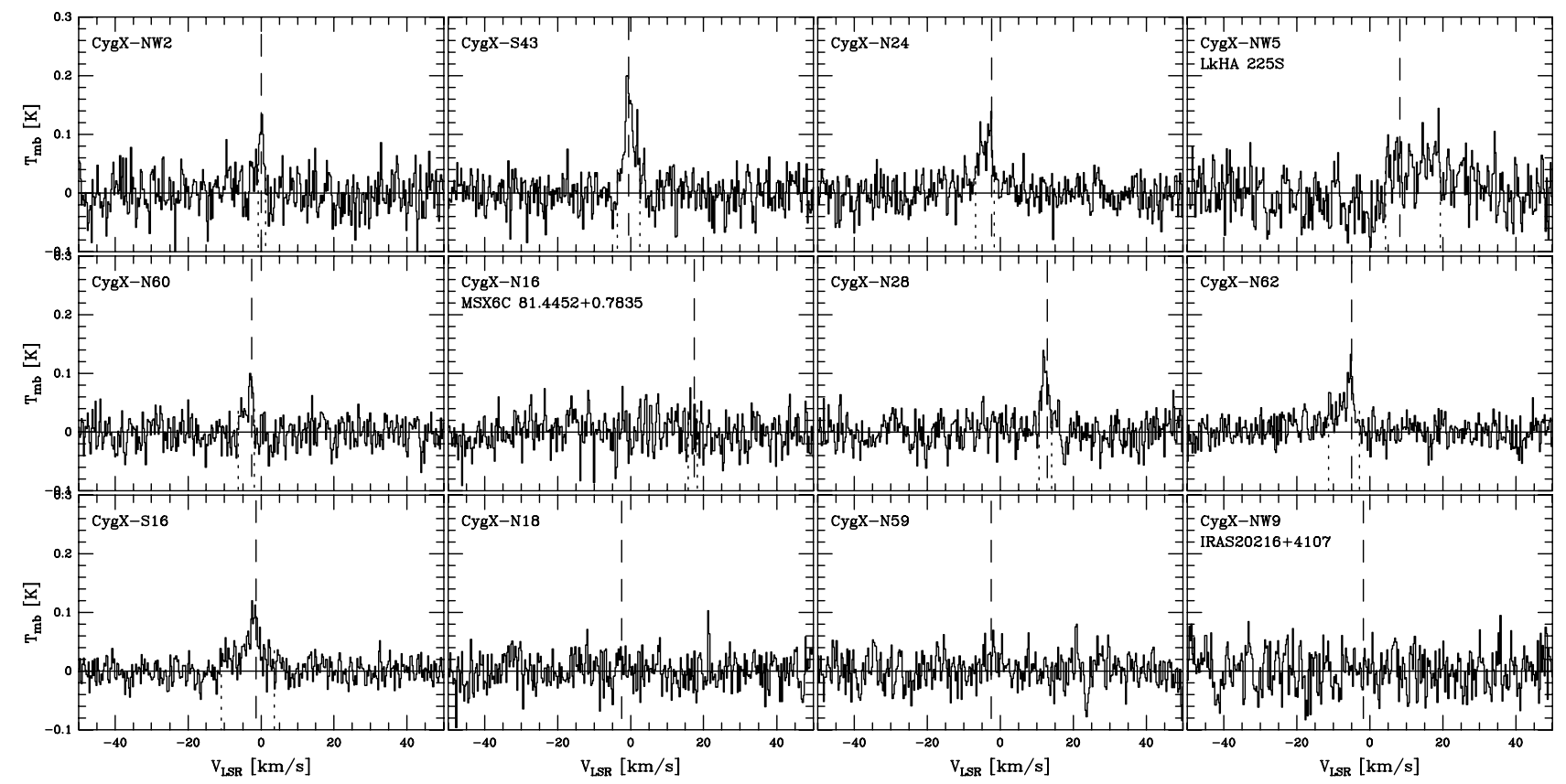

Fig. 15. $\mathrm{SiO}(2-1)$ emission toward 12 additional fragments, ordered by decreasing $M_{1.2} \mathrm{~mm}$. 